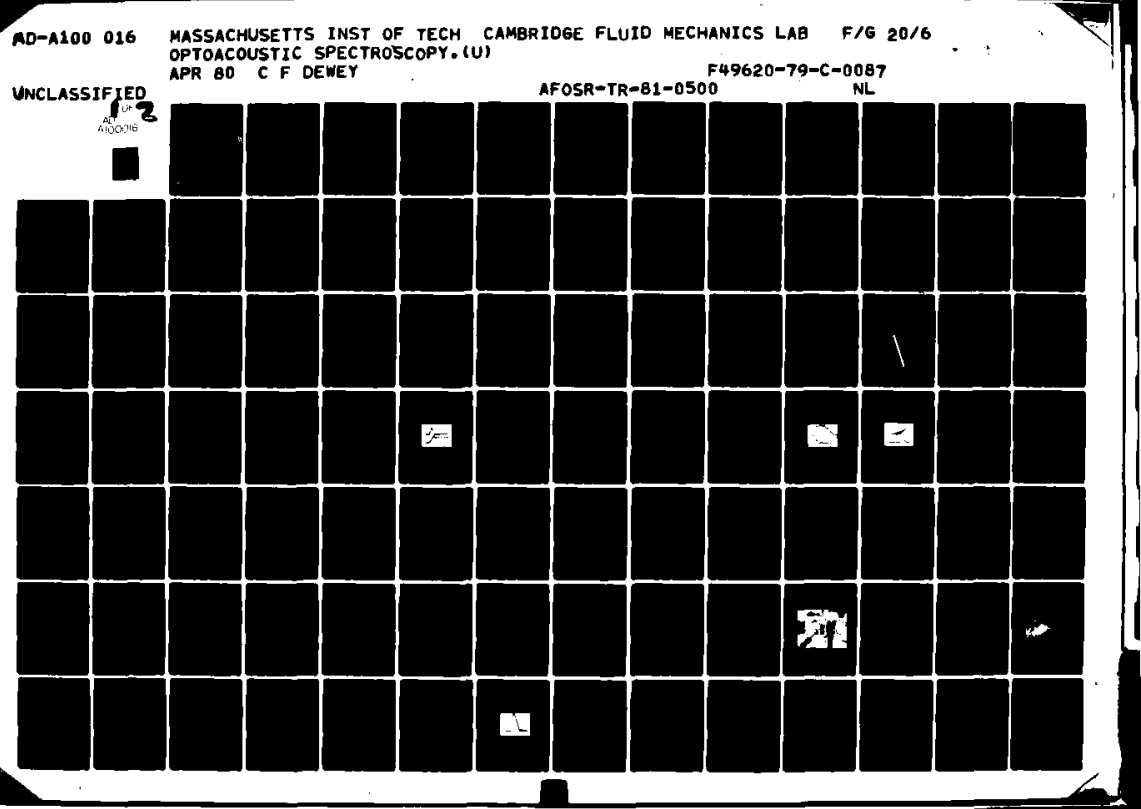




\section{AFOSR-TR- $81-0500$}

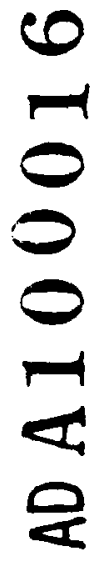

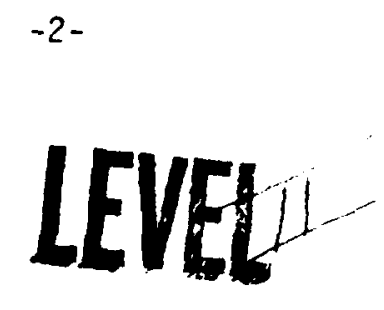

OPTOACOUSTIC SPECTROSCOPY

Final Report

Period Ending 30 April 1980 Contract F49620-79-C-0087

Air Force Office of Scientific Research Bolling Air Force Base, DC Dr. Howard Schlossberg Program Manager

Prepared by C. Forbes Dewey, Jr. Professor of Mechanical Engineering Head, Fluid Mechanics Laboratory
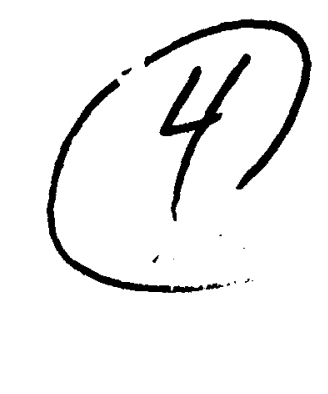

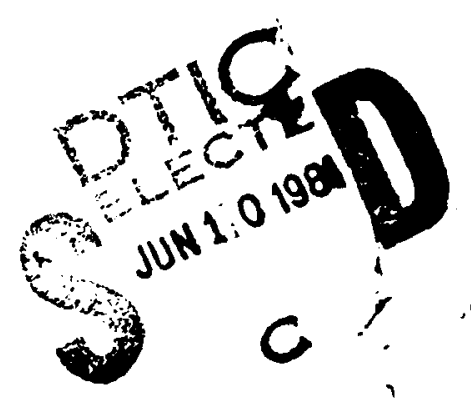

FLUID MECHANICS LABORATORY MASSACHUSETTS INSTITUTE OF TECHNOLOGY ROOM 3-250

CAMBRIDGE, MASSACHUSETTS 02139

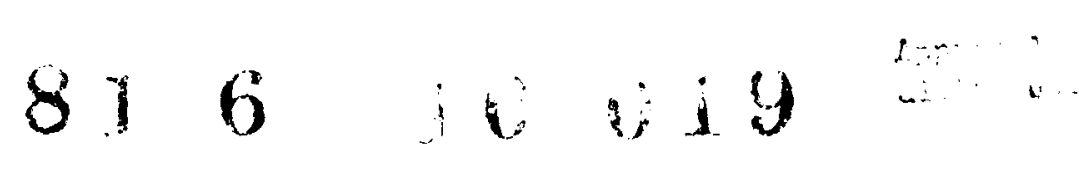


Final Report, Grant F49620-79-C-0087

Air Force Office of Scientific Research

Year Ending 30 Apri?, 1980

\title{
OPTOACOUSTIC SPECTROSCOPY
}

\begin{abstract}
The objective of this program is to develop improved spectroscopic methods for investigating the absorption of gases $i$, the infrared region of the spectrum. During the contract year covered by this Report, we were successful in achievi.g tunaole output from a dye laser oumped by a lead laser, with power levels sufficient to produce tunable infrared radiation by nonlinear mixing.
\end{abstract}

Primary contributors to the program were John H. Flint, James H. Harrison, and Andrei Szilagyi.

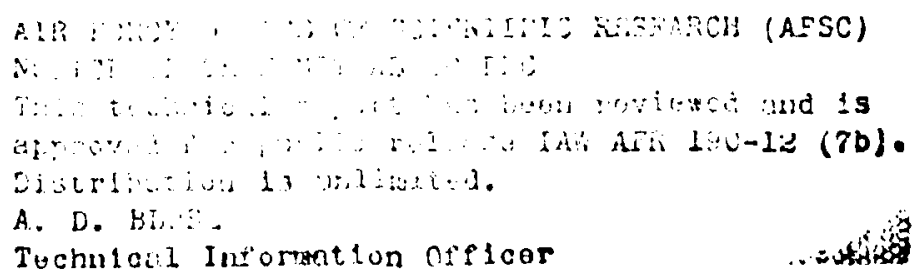


$-4$

TABLE OF CONTENTS

Page

I. Technical Overview of Program

II. Description of the Tunable Infrared Laser System

III. The Lead Vapor Laser

IV. The Dye Laser

V. Nonlinear Mixing in Rotationally-Twinned Crystals

VI. Recommendations for Future Work

Appendix A. Pressure Dependence of the Infrared Laser in Barium Vapor

Appendix B. Difference-Frequency Generation of Tunable Infrared Radiation
5

6

7

8

9

11

12

15

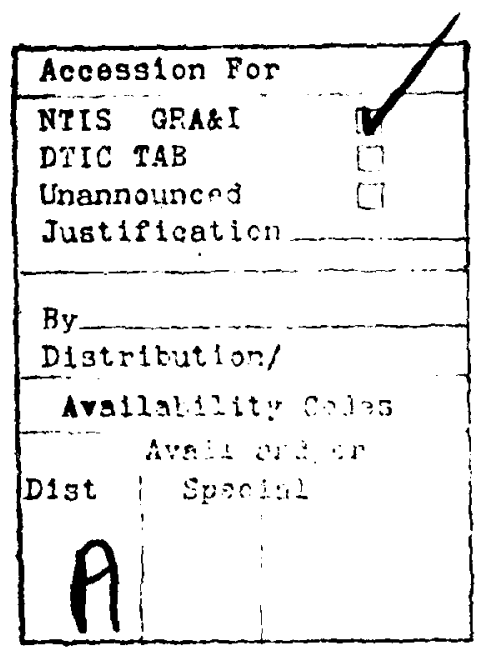


Unclasstfied

SECUFITY CLASSIFICATION OF THIS PAGE (mhan Date Entored) - 1 -

\begin{tabular}{|c|c|c|}
\hline \multicolumn{2}{|c|}{ REPORT DOCUMENTATION PAGE } & \multirow{2}{*}{$\begin{array}{l}\text { READ INSTRUCTIONS } \\
\text { BEFORE CONPLETINC FORM } \\
16\end{array}$} \\
\hline AFOSR-TI- $-81-0500$ & $\begin{array}{l}\text { 2. GOVT ACCESSION NO } \\
4 D-A I C C O\end{array}$ & \\
\hline \multirow{2}{*}{\multicolumn{2}{|c|}{$\begin{array}{l}\text { TITLE iand Subitile) } \\
\text { QPTOACOUSTIC SPECTROSCOPY. }\end{array}$}} & 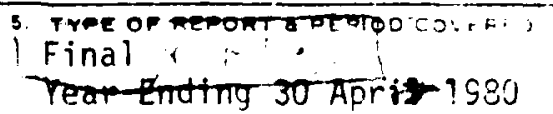 \\
\hline & & B. DERFORMING JRG REPOHT NINUTH \\
\hline \multirow{2}{*}{\multicolumn{2}{|c|}{$\begin{array}{l}\text { 7. AUTMOR(s) } \\
\text { C. Forbes Dewey, Jr.. }\end{array}$}} & 9. CONTRACT OA GRANT NUMESTR'AI \\
\hline & & $F 49620-73-C-0087$ \\
\hline \multirow{2}{*}{\multicolumn{2}{|c|}{$\begin{array}{l}\text { 9. PERFORMING ORGANIZATION NAME AND AODAESS } \\
\text { Fluid Mechanics Laboratory } \\
\text { Massachusetis Institute of Technology } \\
\text { Cambridge, MA } 02139\end{array}$}} & 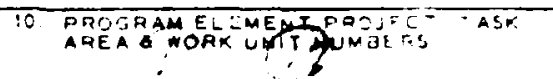 \\
\hline & & $61102 \mathrm{~F} \quad 2301 / 411$ \\
\hline \multirow{2}{*}{\multicolumn{2}{|c|}{$\begin{array}{l}\text { 11. CoNTROLLING OFFICE NAME ANO ADDRESS } \\
\text { Physics Directorate, Air Force Office } \\
\text { of Scientific Research, Building } 410 \text {, Boliting AFB, } \\
\text { D.C. } 20332\end{array}$}} & $\begin{array}{l}\text { 12. AEPOAT DATE } \\
\text { ApriF T9SD }\end{array}$ \\
\hline & & $\begin{array}{l}13 \text { NUMBER OF PASES } \\
113\end{array}$ \\
\hline \multirow{2}{*}{\multicolumn{2}{|c|}{ 14. MONI TORING AGENEY NAME Q ADDRESS(if differont from Controlling Ollice) }} & 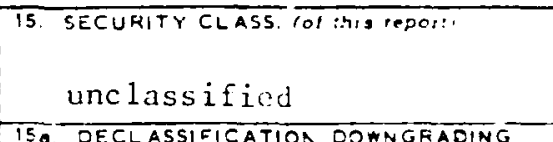 \\
\hline & & $\theta^{\circ}$ \\
\hline
\end{tabular}

15. OISTRIBUTION STATEMENT (OT thio RepQI)

approved tor publis rolguso.?

Q1Btrlbition unzinited.

17. CISTRIBUTION STATEMENT (of the abstract onterod in Block 20, Il dillecent from Report)

1a. SUPPLEMENTARY NOTES

17 KEV wạe (Cunlinue on reverso side II necessary and tdentlfy by block number)

Optoacoustic Spectroscopy, laser spectroscopy, tunable lasers, nonlinear optics. Pb laser, dye laser

25 ABSTRACT (Conilnue on reverab oldo II necossary and ldentlly by block number)

The objective of this program is to develop improved spectroscopic methods for investigating the absorption of gases in the infrared region of the spectrum. During the contract year covered by this Report, we were successful in achieving tunable output from a dye laser pumped by a lead laser, with power levels sufficient to produce tunable infrared radiation by nonlinear mixing. 


\section{TECHNICAL OVERVIEW OF PROGRAM}

The need to determine the absorption characteristics of the atmosphere at specific wavelengths in the infrared region of the spectrum has generated a number of alternative methods of measurement using conventional and laser-based optical sources. This program is directed to the development of an optoacoustic detection system using a wavelength-tunable laser source that is potentially capable of measuring absorption coefficients of $10^{-5}-10^{-7}$ meter ${ }^{-1}$ with an ultimate wavelength resolution of better than $0.1 \mathrm{~cm}^{-1}$.

The method employs a high-power pulsed lead metal vapor laser as the primary optical power source. A portion of this beam is used to pump a dye laser. The remaining lead vapor laser beam is then combined with the dye laser output in a nonlinear optical crystal to produce continuously-tunable infrared radiation. By appropriate choices of the laser dye and nonlinear crystal, infrared radiation may be produced over the wavelength range from $2.5 \mu \mathrm{m}$ to at least $15 \mu \mathrm{m}$.

The infrared laser output consists of a train of optical pulses of about 30 ns duration; the pulse repetition frequency may be varied between $100 \mathrm{~Hz}$ and $2 \mathrm{KHz}$. In our previous research, we have shown that a tunable pulsed infrared laser solirce of this type may be advantageously used in optoacoustic spectroscopy.

There are three principal components to the infrared laser system: the pump laser (lead vapor laser); the dye laser; and the nonlinear crystal. Each of those elements has required substantial research and 
engineering optimization to achieve performance levels suitable for use in a wavelengch-tunable laser system. This report details the progress made in achieving these goals during the contract year ending 30 April 1980.

\section{DESCRIPTION OF THE TUNABLE INFRARED}

\section{LASER SYSTEM}

The difference-frequency method of producing tunable infrared laser radiation is deccribed by the drawing of Fig. 1. $\omega_{3}$ is the frequency of the lead vapor laser operating at a wavelength of $0.7229 \mu \mathrm{m}$. The tunable dye laser $\left(\omega_{1}\right.$ of Fig. 1) can operate between $0.76 \mu \mathrm{m}$ and $1.02 \mu \mathrm{m}$, corresponding to a tunable difference frequency $\left(\omega_{2}\right)$ whose wavelength would cover the range of $2.5 \mu \mathrm{m}$ to $15 \mu \mathrm{m}$.

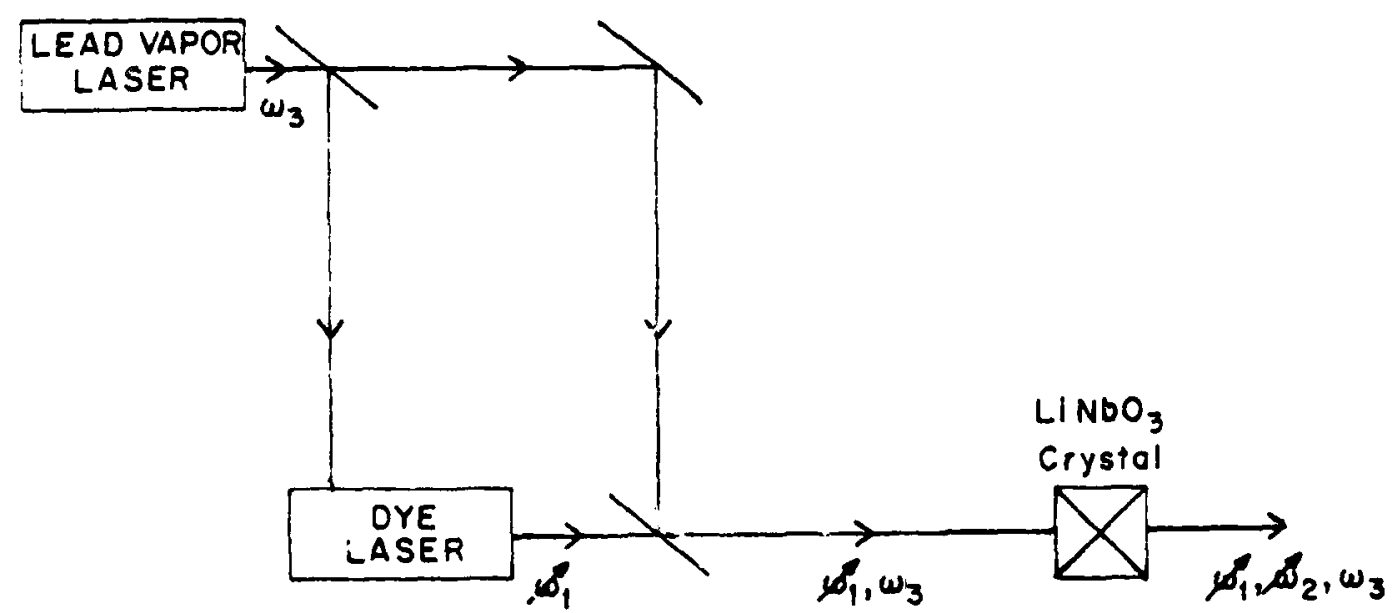

Fig. 1. Difference-frequency generation system. 
We have concentrated our initial experiments on developing efficient dye laser operation in the $800-870 \mathrm{~nm}$ region because the dyes that are available for that spectral region have slightly better efficiency and photochemical stability than the longer-wavelength dyes currently available to us. Once optimum operation has been achieved, it is a re?ativeiy simple matter to change the dyes to ones that would correspond to any infrared wavelengths between $2.5 \mu \mathrm{m}$ and at least $15 \mu \mathrm{m}$.

On the basis of our current results with the lead vapor laser and dye laser, we estimate that tunable infrared laser outputs with average powers on the order of $0.5 \mathrm{~mW}$ may be achieved.

\section{THE LEAD VAPOR LASER}

The lead vapor laser consists of a heated tube containing lead vapor, an optical cavity to promote laser emission from the hot vapor, and a pulsed electrical discharge circuit to produce the population inversion required for laser gain.

Because the exact details of the electrical excitation process producing inversion are not well understood, optimization of the lead vapor laser operating conditions has involved substantial experimentation. This fact, coupled with the corrosive character of lead vapor and the high operating temperature $\left(1050^{\circ} \mathrm{C}\right)$ has made this portion of our program most difficult. 
Appendix B, Chapter 4, describes the lead vapor laser development conducted during this contract period. We have achieved operating times in excess of 25 hours between recharges of the laser tube. Lead vapor laser pulse energies of $125 \mu \mathrm{J} /$ pulse ( $30 \mathrm{~ns}$ ) have been consistently achieved. Average power outputs exceeding $200 \mathrm{~mW}$ at $2 \mathrm{KHz}$ have also been measured.

These characteristics are quite adequate to produce tunable infrared radiation with the difference-frequency system.

We expect that factors of 2-4 improvement in pulse energy may be achieved with further development of this laser. Also, the operating lifetime may be significantiy increased if an alumina tube is used to contain the lead vapor; this improvement was not attempted during the current program because of the prohibitively high costs associated with custom fabrication of the alumina tube.

\section{THE OYE LASER}

Appendix $A$ presents detailed results obtained with our new "zcavity" dye laser configuration. We have achieved excellent beam quality and pulse-to-pulse stability from the dye laser at instantaneous levels in the dye beam of about 50 watts. Substantial power improvements are possible with improved quality cavity tuning elements.

The difference-frequency system we have adopted is particularly attractive for optoacoustic work. The high repetition rate of the 
laser pulses. coupled with the high average power levels, is ideal fur investigations of atmospheric absorption. However, this system is very difficult to achieve in practice because the energy (and instantaneous power levels) of each individual pulse is low.

The "z-cavity" dye laser we have designed and successfully operated is a significant improvement over previous dye laser designs when the requirements of the difference-frequency system are considered. Although the initial results achieved during this contract period showed fairly low slope efficiencies of $2 \%$, we expect that a very modest amount of work and the use of higher-quality dispersive components in the dye laser cavity will lead to substantial improvements.

The current dye laser power levels are quite sufficient to produce nonlinear generation of tunable infrared radiation.

\section{NONL INEAR MIXING IN ROTATIONALLY-}

\section{TWINNED CRYSTALS}

During the year, A. Szilagyi continued his work on rotationallytwinned crystals as desirable alternatives to conventional birefringent materials for nonlinear optical mixing.

Although the current objective of achieving tunable infrared radiation in the $2.5 \mathrm{\mu m}-4.5 \mathrm{um}$ spectral region encompassing HF-DF laser operation may be achieved using $\mathrm{LiNbO}_{3}$ and $\mathrm{LiIO}_{3}$ crystals, the full potential tuning range of the difference-frequency system cannot be 
achieved without new crystals that operate over larger segments of the infrared.

In our previous research under this contract, we have demonstrated experimentally and theoretically that rotationally-twinned $43 \mathrm{~m}$ crystals are very attractive for this type of mixing process. The major stumbling block to widespread application of this approach is the difficulty of obtaining crystals with favorable twin spacing.

During the early part of the contract year, we continued to examine as-grown CiTe crystals from commercial sources to see if fortuitous twin spacings were obtained frequently enough to make selection of suitable crystals feasible. Our conclusion is that this method is indeed feasible if subsequent cutting, polishing, and optical contacting is used to create stacks of twinned plates oriented to produce a cooperative effect. Because CdTe is very soft and difficult to hand?e mechanically, particularly during manipulations such as stacking, this method of implementing the rotational twin concept in nonlinear optics is very difficult.

During the latter portion of the year, attention was given to novel methods of controlling twin formation during growth. Several methods involving mechanical and thermal modification of conventional Bridgman growth methods were considered. Because the mechanisms causing twin formation are not understood, the feasibility of these proposals could not be assessed without experimental trials. And such experiments, because of their difficulty and complexity, were not possible within the fiscal and temporal constraints of this program. 
For these reasons, we have begun a cooperative study with Prof. A. Witt of the Materials Sciences Department to identify the fundamental mechanisms leading to twin formation during crystal growth. Within this effort, the experimental resources will be available to explore methods of producing optical crystals with controlled twin spacing that will be suitable for various important nonlinear optical processes.

\section{RECOMMENDATIONS FOR FUTURE WORK}

The full potential of the difference-frequency method for producing a tunable infrared optical source suitable for optnacoustic spectroscopy has yet to be realized. Although the major components of a successful system have been demonstrated (lead laser, dye laser, phase-matched nonlinear optical crystal), the final combination of these elements that was to be completed with additional funding was not accomplished because the additional funding was never received. It is recommended that this promising technique be pursued further. 


\title{
APPENDIX A
}

\section{Pressure Dependence of ine Infrared Laser Lines in Barium Vapoi}

\author{
M MADIGAN, L. O. HOCKER, J. H. FIINT, AND C. F. DEWEY, JR
}

\begin{abstract}
We have studied nine laser transitions in Ba vapor at wave. lengths between 1.13 and $3.05 \mu \mathrm{m}$. Three of these laser lines have rot been reported previuusly. The relative intensities of the four strongest trancitions $(1.13,1.50,2.55$, and $2.92 \mu \mathrm{m})$ vary with buffer gas pressure, sugesting that colligional deexcitation of the $6 p^{1} P_{1}$ state occurs at higher pressure and is the main mechanism populating the upper states of the 2.55 and $2.33 \mu \mathrm{m}$ lines.
\end{abstract}

$M$ ETAL vapor lasers have demonsirated a potential for high power and efficiency [1]-[3]. The efficiency re. sults from a favorable ratio of laser photon energy to energy of the upper laser level and a large cross section for electron excitation from the ground state into an upper laser state.

Laser amission in barium vapor was first observed by Cahuzac [4]. Who identified twenty emission lines. Subsequent work by others $[31.15]$ have demonstrated average power levels of several watts and electrical to optical conversion efficiencies as high as 0.54 percent. Although the dominant laser transitions produce radiation at 1.50 and $1.13 \mu \mathrm{m}$, substantial emission has been observed at several other wavelengths. We have found collisional deexcitation to be important at high buffer gas pressures. The deexcitation quenches some emission lines, but anparently is the excitation mechanism for others.

Manuscript rocetved August 15, 1980. This work was supported by the Air Force Weapons Laboratory and the Air Force Office of Scientific Research under Contract F29601-78-C-0029.

M. Madigan was with the Department of Mechanical Engineering, Massachusetts Institute of Technolog3, Cambridge, MA 02139. He is now with Optical Coating Laboratories, Santa Rosa, CA 955402.

L. O. Hocker was with the Department of Mechanical Engineering. Massachusetts Institu:e of Technology. Cambridge, MA 02139. He is n:lw with Sea Data Corporation, Newton, MA 02158

J. H. Flint and C. Dewey, Jr. are with the Departmen: of Mechanical f.ngineering. Massachusetts Institute of Technology. Cambridge. MA $n 2139$
Fig. $I$ is an energy level diagram that includes the eleven dominant laser lines that we observed in Ba vapor. Table ! lists these lines and our assignments of the upper and lower laser levels; we are confident of the assignments of the firs: nine lines, but the final two assignments are less certain.

Fig. 2 is a partial energy level diagram that includes thuse transitions whose intensities exhibii a dependence on buffer gas pressure. The Bom approximation predicts that the ' $P_{1}$ and ${ }^{3} P_{1}$ levels will be pumped predominantly. We observed laser emission on four of the six transitions allowed from these states. inciuding one line that has not been observed previously. This line, and two other new laser lines previously seen onl in spon. taneous emission, are marked with asterisks in Table I

For this study, an alumina tube-- $76 \mathrm{~cm}$ long. $4.2 \mathrm{~mm} 1 \mathrm{ID}$. and fitted with Brewster-angle sapphire windows was employed as the discharge vessel. An oven heated the central $40 \mathrm{~cm}$ of the tube to approximately $950^{\circ} \mathrm{C}$ so that the Bariuir: density was independent of repetition rate. Excitation was provided by discharging a $1.0 \mathrm{nF}$ capacitor through the laser using an E. G. \& G. 1802 thyratron. The capacitor was resonantly charged through a high-voltage diode and a $10 \mu \mathrm{H}$ induc. tor from a $10 \mathrm{kV}$ power supply. The maximum repetition rate was limited to $2 \mathrm{kHz}$ by the thyratron trigger. The optical cav. ity was formed by a 1 in gold coated flat nd a $i$ in uncosted sapphire flat.

Figs. 3 and 4 report the results of our pressure dependence studies; only the four sirongest lines are shown for clanty. There are several interesting features which indicate that collisional processes dominate the coupling of these low lying multiplet levels. Pressures were measured with either a mercury or an oil manometer, and the intensities are simply the defector voltages uncorrected for the PbSe detector responsivity or 
$-13-$

IEEE JOURNAL OF QUANTUM ELECTRONICS, VOL. QE-16, NO. 12, DECEMBER IFo

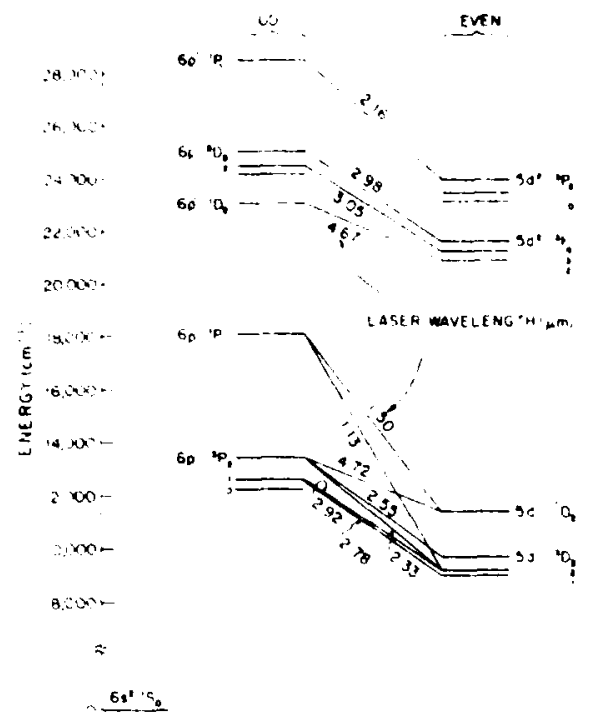

Fig. 1. Energy levels of obeorved laser lines in barium. The aedignments of the two lines $2: 4.67$ and $4.72 \mu \mathrm{m}$ are less certain than the other nine assignments.

TABLE 1

ORSzRED LASER LINES IN Ba

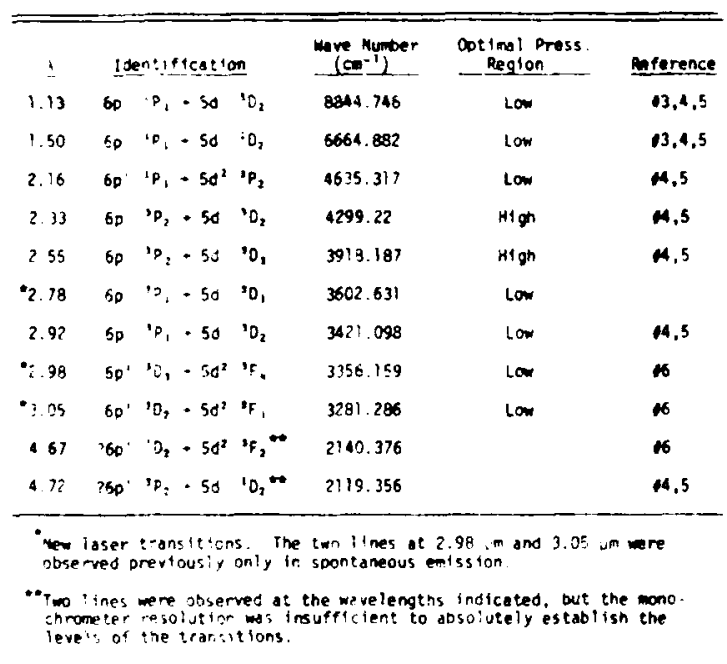

monochromator efficiency. The "kink" in the $2.92 \mu \mathrm{m}$ output at 4 tor of helium may be an idiosyncracy of our particular laser cavity and is shown only as a tribute to its persistence.

The most striking feature of the pressure-dependence curves is that they have almost identical forms with both heilum and neon as buffer gases, with the exception that the neon buffer gas pressure required to produce a diven wavelength distribution is about a factor of three higher than the correaponding pressure for helium. This is a strong indication that this is a colir. sional effect, especially since the pressure ratio of throe corre. sponds relatively closely to the ratio of the thermal velocities of the buffer gases. Ore would expect an He : Ne pressure ratio of 2.24 if the collisional processes scaled according to harcsphere collision cross sections anc depended only on the atomic diameter and velocity of the buffer gas.

Both the 2.55 and $2.33 \mathrm{~mm}$ (not shown) transitions oscillate only at high buffer gas preasure. The upper state of both lines

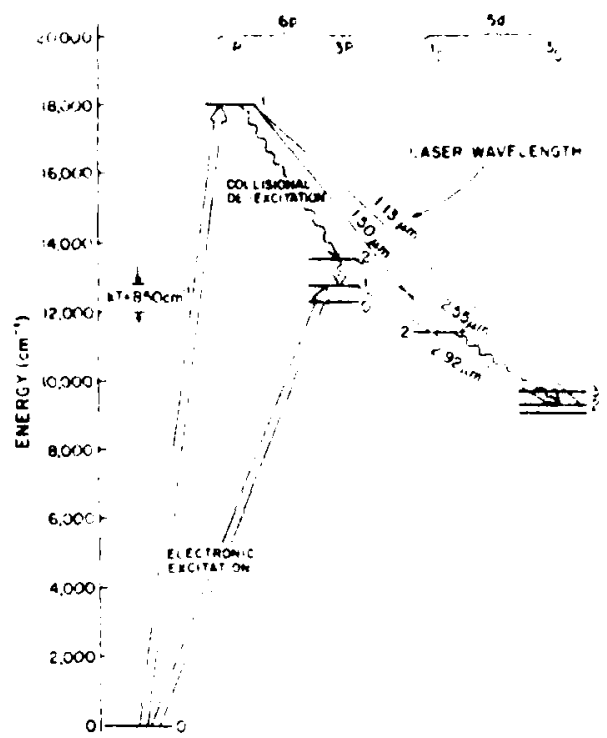

Fig. 2. Partial energy level diagram shuwing proposed eiectronic excita. tion and collisional doexcitation mecianism.s.

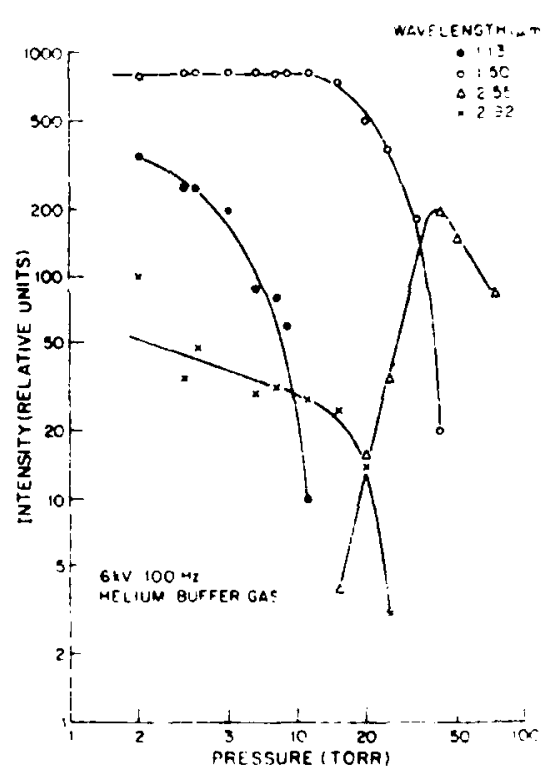

Fig. 3. Laser intensity variation in helium.

is the $6 p^{3} P_{2}$ level which does not have an electronic transition to the ground state and, accordingly, is most probably not pumped dirertly by electron collisions. It is difficuit to justify a collisional promotion of an atom in the $6 p^{3} p_{1}$ ieve! to the $6 p^{3} P_{2}$ level because the latter is about $i k T$ higher in energy. One may therefore conclude that the population of the $6 p^{3} P_{2}$ level comes from collitional deexcitation of the $6 p^{1} P_{1}$ level, the upper state of the two strongent lines at 1.13 and $1.50 \mu \mathrm{m}$.

Colliatonal coupling can also account for the pressure depen dence of the $1.13,1.50$, and $2.92 \mu \mathrm{m}$ transitions. Deexcitation of the $S d{ }^{1} D_{2}$ leve? to the $5 d^{3} D_{2}$ level will enhance the $1.50 \mu \mathrm{m}$ transition at the expense of the 1.13 and $2.92 \mu \mathrm{m}$ transitions leading to the persistence of the $1.50 \mu \mathrm{m}$ line at relatively high pressure. One would also expect the $2.92 \mu \mathrm{m}$ 


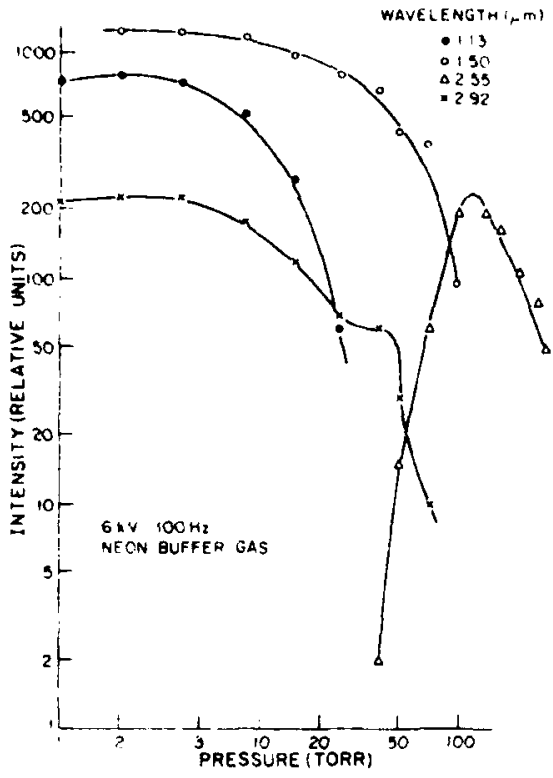

Fig. 4. Laser intensity variation in neon.

transition to lase at a higher pressure than the $1.13 \mu \mathrm{m}$ transition because collisional relaxation of the $6 p^{1} P_{1}$ level to the $6{ }^{3} P_{1}$ will inhibit the $1.13 \mu \mathrm{m}$ line while benefiting the 2.92 $\mu \mathrm{m}$ line.

Collisional effects will be in evidence only if the rate of deexcitation is comparable to the inverse of the pulse length, $\tau_{p}$. If the buffer gas means thermal velocity is $v$ and the buffer gas number density is $n$, then the deexcitation cross section $\sigma$ may be estimated from the relation

$$
\sigma \approx\left(n v \tau_{p}\right)^{-1}
$$

The temperature and pulse length were approximately $950^{\circ} \mathrm{C}$ and $20 \mathrm{~ns}$, respectively. The He number density at which the collisional deexcitation $6 p^{1} P_{1} \rightarrow 6 D^{3} P_{2}$ orriles corresponds to a pressure of about 20 torr; at this pressure, the intensity of the $2.55 \mu \mathrm{m}$ laser line originating from the $6 p^{3} P_{2}$ level has reached 10 percent of its maximum value. The resulting estimate of $\sigma_{\mathrm{HaBa}}$ is $1.3 \times 10^{-15} \mathrm{~cm}^{2}$. In a similar manner, we may associate the initial reduction of intensity of the :.1 $3 \mu \mathrm{m}$ line with collisional population of its lower laser level va $5 d{ }^{1} D_{2} \rightarrow 5 d{ }^{3} D_{2}$ deexcitation; this iransition has decreasec 10 percent in intensity at 8 torr $\mathrm{He}$, yielding a cross section $\sigma_{\mathrm{Ho}_{0} \mathrm{Ba}}$ of $2.5 \times 10^{-15} \mathrm{~cm}^{2}$. The gas kinetic He-Ba cross sec. tion is $4.2 \times 10^{-15} \mathrm{~cm}^{2}$; thus, our estimates of the two deexcitation cross sections correspond to $\frac{1}{3}$ and $\frac{3}{5}$ of the gas. kinetic cross section.

Collisional effects are clearly important, even for moderatepressure operation of the barium laser, and with sufficient understanding of these processes, it may be possible to significantly improve the efficiency of this laser. Metal vapor lasers have great potential for high power and high efficiency, but it will be difficult to fully realize this potential without an increased understanding of the details of the collisional excitation and deexcitation mechanisms.

\section{REFERENCES}

[1] A. A. Laev and G. Y. Lemmerman, "Investigation of a copper vapor pulsed laser at elevated powers," Sov. J. Quantum Electron., voi. 7, pp. 799-801, 1977.

[2] A. A. lsaev and M. A. Kazaryan, "Investigation of pulse coppervador lases," Sov. J. Quantum Electron, vol. 7, pp. 253-255, 1977.

[3] B. G. Bricks, T. W. Karras, and R. S. Anderson, "An investigation of a discharge heated barium laser," $J$. Appl. Phys., vol. 49, pp. 38-40, 1978.

14] P. Cahuzac, "Nouvelles raies laser infrafouges dans la vapeur de baryum." Phys. Lett. vol. 32A. DD. 150-151. 1970.

(5) A. A. Isaev, M. A. Kazaryan, S. V. Markrova, and G. G. Petrash, "Investigation of pulse infrared stimulated emission from barium vapor," Sov. J. Quantum Electron., vol. 5, pp. 285-287, 1975.

[6] H. P. Palenus. "The establishment of the $5 \mathrm{~d}^{2}{ }^{3} \mathrm{~F}$ and ' $S$ levels in Bal," Phys. Lett., vol. 56A, pp. 451-452, 1976.

[7] C. E. Moore, Nat Bur. Standards Circular 467, vol. 3, p. 132,1949 


\author{
APPENDIX B \\ DIFFERENCE-FREQUENCY GENERATION \\ OF TUNABLE INFRARED RADIATION
}

by

James Harrison

M.S. Thes is

Massachusetts Institute of Technology

December 1980 


\title{
DIFFERENCE-FREQUENCY GENERATION
}

OF TUNABLE INFRARED RADIATION

by

\section{JAMES HARRISON}

\begin{abstract}
Submitted to the Departinent of Electrical Engineering on December 5,1980 in partial fulfillment of the requirements for the Degree of ilaster of Science in Electrical Engineering
\end{abstract}

\section{ABSTRACT}

A difference-frequency system is being developed as a source of pulsed radiation in the 2.5-4.5 um spectral region. It will be capable of operation at pulse repetition frequencies as high as $2 \mathrm{KHz}$. Applications of this system include opto-acoustic spectroscopy of the atmosphere.

Two primary sources, a lead vapor laser and a tunatle dye laser, have been developed. These lasers will stimuiate a phase-matched lithium niobate crystal to produce the infrared difference-frequency. The lead vapor laser operates at $.7229 \mathrm{um}$ at pulse repetition frequencies up to $2 \mathrm{KHz}$. The output pulse width is about $30 \mathrm{~ns}$ and $125 \mathrm{ij}$ pulse energies have been achieved. The lead vapor laser outnut will be divided between the mixing crystal and the dye laser. Pumped by the lead vapor laser, the dye laser has been operated at wavelengths beyond $.89 \mathrm{um}$ at linewidths well below that required for efficient mixing. A detailed analysis of the dye laser "z-cavity" is presented.

Temperature control of the mixing crystal will be employed to achieve $90^{\circ}$ phase-matching. The degree of temperature control necessary for efficient mixing has been calculated and demonstrated. The complete system is expected to operate at a peak power above $10 \mathrm{~W}$ and an average power above $.5 \mathrm{~mW}$.

Thesis Supervisor: Dr. C. Forbes Dewey, Jr.

Title: $\quad$ Professor of Mechanical Engineering 


\section{ACKNOWLEDGEMENTS}

My deepest thanks to Professor Dewey for the opportunity, for his guidance, and for all he has taught me and shown me.

John H. Flint has contributed greatly to this project ard deserves both credit and thanks.

I wish also to acknowledge Richard R. Fenner for his expert technical assistance.

This research was sponsored in part by the Air Force Office of Scientific Research and the Air Force Weapons Laboratory under Contracts F92601-78-C-0029 and F49620-79-C-0087. 


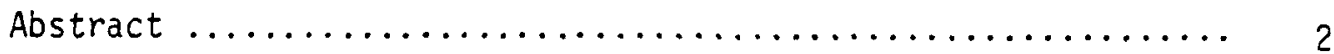

Acknowledgements $\ldots \ldots \ldots \ldots \ldots \ldots \ldots \ldots \ldots \ldots \ldots \ldots \ldots \ldots \ldots \ldots$

List of Figures $\ldots \ldots \ldots \ldots \ldots \ldots \ldots \ldots \ldots \ldots \ldots \ldots \ldots \ldots \ldots \ldots \ldots \ldots \ldots \ldots \ldots$

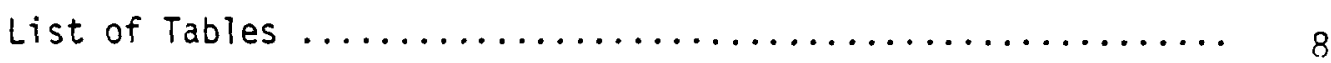

1. INTRODUCTION $\ldots \ldots \ldots \ldots \ldots \ldots \ldots \ldots \ldots \ldots \ldots \ldots \ldots \ldots \ldots \ldots \ldots \ldots$

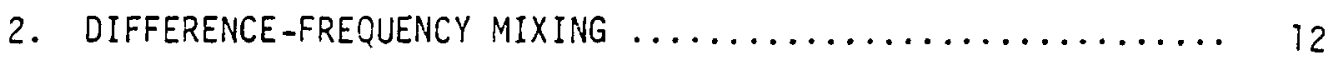

2.1 Fundamental Characteristics of the Process ........ 12

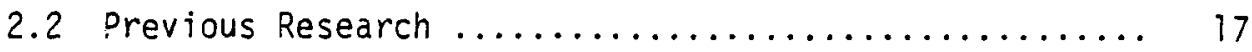

2.3 Configuration of the Present System $\ldots \ldots \ldots \ldots \ldots . . .20$

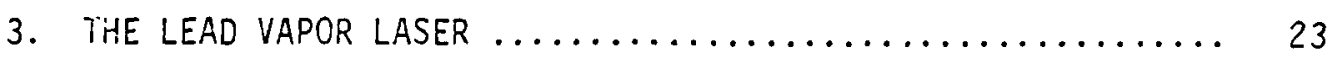

3.1 Laser Emission at $.7229 \mu m \ldots \ldots \ldots \ldots \ldots \ldots \ldots \ldots . \ldots \ldots$

3.2 Description of the Lead Vapor Laser ............. 25

3.3 The Pulse-Forming Circuit .................. 28

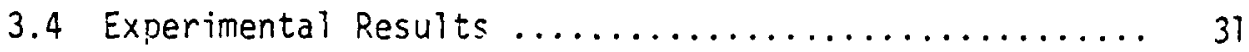

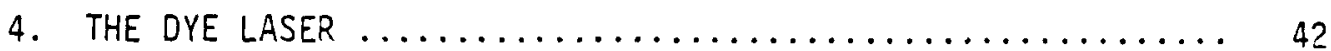

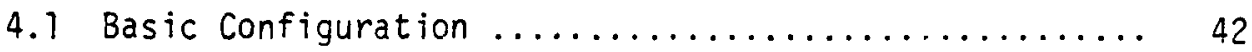

4.2 Four-Mirror Equivalent Cavity .............. 47

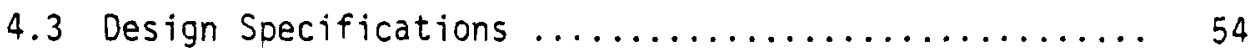

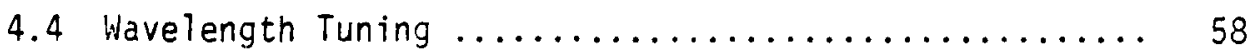

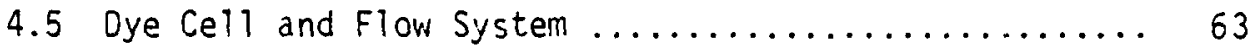

4.6 Experimental Results ..................... 71 


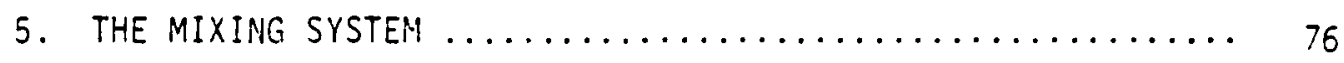

5.1 Properties of Lithium Niobate ............... 76

5.2 Temperature Control of the Mixing Crystal ........ 77

5.3 The Optical System $\ldots \ldots \ldots \ldots \ldots \ldots \ldots \ldots \ldots \ldots \ldots .63$

5.4 Theoretical Conversion $\ldots \ldots \ldots \ldots \ldots \ldots \ldots \ldots \ldots \ldots \ldots$

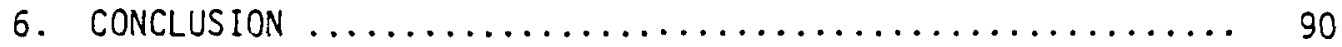

APPENDIX A. Detectivity of Crystal Spontaneous Parametric

Emission and Crystal Calibration ............ 94

REFERENCES $\ldots \ldots \ldots \ldots \ldots \ldots \ldots \ldots \ldots \ldots \ldots \ldots \ldots \ldots \ldots \ldots \ldots \ldots \ldots$ 


\section{LIST OF FIGURES}

Fig. 1 Difference-frequency generation system

Fig. 2 Partial energy level diagram of $\mathrm{Pb}$

Fig. 3 Lead vapor laser configuration

Fig. 4 Lead vapor laser discharge circuit

Fig. 5 Lead vapor laser current and voltage waveforms

Fig. 6 The "barbell" laser tube

Fig. 7 Lead vapor laser current and output waveforms

Fig. 8 Lead vapor laser voltage and output waveforms

Fig. 9 Z-cavity with prism

Fig. 10 Z-cavity with diffraction grating

Fig. 11 Lens equivalent of the z-cavity

Fig. 12 Cavity representation

Fig. 13 Beam transformation by a lens

Fig. 14 Isoceles prism at minimum deviation

Fig. 15 The dye cell

Fig. 16 Exploded view of dye cell

Fig. 17 Dye flow nozzle

Fig. 18 Dye cell channel

Fig. 19 Dye laser output energy variation with input pump energy

Fig. 20 Dye laser output waveform

Fig. 21 Calculated phase-matching temperatures ( $880 \mathrm{~nm} \leq \lambda_{1} \leq 1020 \mathrm{~nm}$ )

Fig. 22 Calculated phase-matching temperatures (890 nm $\leq_{1} \leq 900 \mathrm{~nm}$ )

Fig. 23 Relative mixing efficiency as a function of deviation from phase-matching temperature 
Fig. 24 Schematic diagram of the complete optical system

Fig. 25 Alternative mixing system

।

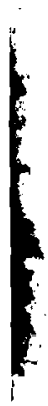




\section{LIST OF TABLES}

Table 1. Dye laser design specifications

Table 2. Absorption data for dye solutions

Table 3. Maximum allowable dye laser linewidths for efficient mixing $(\Delta k \leq \pi / 2)$ 


\section{INTRODUCTION}

Over the past decade many researchers have concentrated on the development of continuously-tunable infrared laser sources. One spectral region of particular interest is the near infrared from $3 \mathrm{jm}$ to $5 \mathrm{jm}$ in wavelength. In this region work continues on both primary sources, principally F-center and semiconductor lasers, and frequency converters employing Raman scattering or multiple-wavelength mixing. This thesis deals with the development of a three-wave (difference-frequency) mixing system.

Much of this research has been motivated by the need for high resolution spectroscopic investigation of the atmosphere. Many molecules that occur as trace atmospheric constituents exhibit absorption bands in the infrared. In the 3-5 um spectral region these inciude $\mathrm{O}_{3}, \mathrm{CH}_{4}, \mathrm{C}_{4} \mathrm{H}_{10}, \mathrm{NO}_{2}, \mathrm{HCl}, \mathrm{SO}_{2}, \mathrm{CO}_{2}$ and $\mathrm{CO}$. This region is of particular interest because the relative lack of absorption exhibited by the atmosphere from 3-5 um makes it a potential window for the propagation of high power laser sources. For this reason it is important to study the absorption characteristics with a high degree of sensitivity. A tunable laser source operating at linewidths narrower than those of the absorption bands is the key to this study. It would also have immediate application in atmospheric pollution monitoring devices as well as potential applications including the investigation of the molecular structure of various hydrocarbons and experimental laser chemistry. 
Optoacoustic spectroscopy appears to be the most sensitive technique for absorption measurements. In a typical system, a pulsed optical source is incident on a cell containing the absorbing sample. The energy absorbed by the sample creates an acoustic signal at the pulse repetition frequency of the source. The signal power is proportional to the average power of the source. Optoacoustic spectroscopy is probably the method of choice for measurements of trace species occurring at concentrations below 1 part in $10^{7}$ and absorption coefficients of less than $10^{-7} \mathrm{~cm}^{-1}$.

These considerations result in the further constraint of the desired infrared laser source to operation in a pulsed mode at high average power with narrow linewidth and a broad tuning range. This thesis reports on the development of such a source that will operate over the 2.5-4.5 un spectral region. A lead vapor laser and a near infrared dye laser have been constructed as part of a differencefrequency system that, including a lithium niobate crystal, will be capable of fulfilling the requirements for a tunable infrared source.

In the following Chapter, the fundamental relationships governing difference-frequency mixing are presented along with a brief comparison of this system with other potential sources of tunable infrared radiation. The description of the present system follows a review of publications describing results obtained on analogous tunable infrared laser systems. Chapters Three through Five deal with the primary system components: the lead vapor laser, the dye laser and the mixing system. The lead vapor and dye laser sections contain discussions of 
the details of design, construction and operation. Chapter five is concerned with the phase-matching requirements of the crystal as well as the optical configuration necessary to perform the mixing. In the final Chapter, the present state of the system is discussed along with several suggestions for further research. 


\section{DIFFERENCE-FREQUENCY MIXING}

In this Chapter, the theoretical and practical background of the system under development is detailed. The fundamental relations underlying the difference-frequency mixing process are presented in Section 2.1. Section 2.2 lists the inherent advantages of this process over alternative sources of tunable infrared radiation and reviews the literature concerning difference-frequency systems in the spectral region of interest. The final Section outlines the specifics of the system.

\subsection{Fundamental Characteristics of the Process}

Difference-frequency generation is a three-wave mixing process. It results from the nonlinear polarization induced in certain crystals by an intense electromagnetic field. The dielectric polarization $\bar{P}$ induced by an electric field $\bar{E}$ can be found from the series expansion

$$
\bar{P}=x^{(1)} \cdot \bar{E}+x^{(2)} \cdot \bar{E} \cdot \bar{E}+x^{(3)} \cdot \bar{E} \cdot \bar{E} \cdot \bar{E}+\ldots
$$

where $x^{(n)}$ represents the dielectric susceptibility tensor of the material of rank $n+1$ and order $n$.

An electric field $\bar{E}$ composed of two waves at frequencies $\omega_{i}$ and $\omega_{2}$ where

$$
\bar{E}=\bar{E}_{1} \cos \omega_{1} t+\bar{E}_{2} \cos \omega_{2} t
$$


will induce a first-order nonlinear polarization which may be obtained from the polarization relation

$$
\bar{p}^{(2)}=i^{(2)} \cdot \bar{E} \cdot \bar{E}
$$

In tensor notation, this equation becomes

$$
\begin{aligned}
& \bar{p}_{i}^{(2)}=\frac{1}{2} \sum_{j k} r_{i j k}^{(2)} \cdot\left(E_{I_{j}} \cdot E_{I_{k}}\right)\left[1+\cos 2 i_{i} t\right]+ \\
& \left(E_{2} ; \cdot E_{2_{k}}\right)\left[1+\cos 2 \omega_{2} t\right]+ \\
& \left(E_{1} \cdot E_{2}\right)\left[\cos \left(\omega_{1}+\dot{i}_{2}\right) t+\cos \left(\omega_{1}-\omega_{2}\right) t\right]+ \\
& \left(E_{2 j} \cdot E_{1_{k}}\right)\left[\cos \left(\omega_{1}+\omega_{2}\right) t+\cos \left(\omega_{1}-\omega_{2}\right) t\right] !
\end{aligned}
$$

Equation 4 demonstrates that materials with a non-vanishing second-order susceptibility $x^{(2)}$ may exhibit a constant polarization as well as polarization waves at the second harmonics $\left[2 w_{1}, 2 w_{2}\right]$ and at the sum and difference frequencies $\left[\left(w_{1}+\omega_{2}\right),\left(\omega_{1}-w_{2}\right)\right]$. Symmetry considerations dictate that $x^{(2)}$ will be nonzero orily for non-centro-symmetric crystals. It is important to note that the magnitudes of the components of the susceptibility tensor $x^{(n)}$ decrease rapidly with increasing order of nonl inearity $\left(x^{(1)}: x^{(2)}: x^{(3)} \cong\right.$ $1: 10^{-3}: 10^{-15} \mid$.

It is evident that, by varying the frequency of one or both of the components of the incident electric field $\bar{E}\left(\nu_{1}, \nu_{2}\right)$, the frequencies 
of the components of the resultant polarization waves $\bar{p}^{(2)}\left(2 w_{1}, 2 w_{2}\right.$, $\left.w_{1}+w_{2}, w_{1}-w_{2}\right)$ can be varied. The existence of dye lasers that are tunable in the visible and near infrared spectral regions makes difference-frequency mixing an attractive process for the generation of tunable infrared radiation.

A difference-frequency system requires two input electromagnetic fields: one at frequency $\omega_{3}$ (the pump frequency) and a second at frequency $\omega_{1}$ (the signal frequency). Fields at these frequencies are mixed to generate an output field at frequency is where

$$
\omega_{2}=\omega_{3}-\omega_{1}
$$

Each photon at the difference-frequency $w_{2}$ may be viewed, by analogy, to have been generated by the "dissociation" of one pump photon at into a photon at $\omega_{1}$ and a photon at $\omega_{2}$. In the mixing process, energy is transferred from the pump field to both of the lower-frequency fields. The total energy in the three electromagnetic fields is conserved.

In order to maximize the efficiency of the conversion process, the phase velocities of the three mixing waves must be related in such a way that the waves at $w_{2}$ induced at different points within the mixing crystal interfere constructively. This will be the case when

$$
\Delta \bar{k} \equiv \bar{k}_{3}-\bar{k}_{2}-\bar{k}_{1}=0
$$


where $\bar{k}_{i}$ are the relevant wave vectors

$$
\left|\bar{k}_{i}\right|=\frac{n \omega_{i}}{c}
$$

Under this condition, the mixing crystal is said to be "phase-matched" for the particular mixing process described by the frequencies $\omega_{1}, \nu_{2}, \nu_{3}$.

In general, the index of refraction $n$ is a function of frequency (dispersion), polarization (for anisotropic media), and temperature. True phase-matching (where Eq. (6) holds) is only possible in certain crystals that exhibit birefrigence. It is accomplished by careful control of crystal orientation, the polarizations of the input beams, and, in cases where the refractive indices show a strong temperature dependence, crysta 1 temperature.

The phase-matching relation represented by Eq. (6) may be viewed as the conservation of momentum condition for the mixing process just as Eq. (5) represents the conservation of energy. The power $P_{2}$ generated at $\omega_{2}$ depends strongly on the phase-mismatch $|\Delta \bar{k}|$ as defined by Eq. (6)

$$
P_{2} \propto \operatorname{sinc}^{2}\left(\frac{\mid \Delta \bar{k}_{i} i}{2}\right)
$$

where $\ell$ is the crystal length.

Typically a conversion efficiency $P_{2} / P_{3}$ of less than a few percent is achieved and pump depletion is negligible. In addition, double refraction and diffraction effects can usually be ignored. Under these conditions, and assuming the incident fie: -onsists of perfectly 
phase-matched $(!, \bar{k})=0)$ Gaussian Deams, the power $P_{2}$ generated at is is approximately (in MKS units) ${ }^{3}$

$$
P_{2}=\frac{4 \omega_{2}^{2} d^{2} 2^{2} P_{3} P_{1}}{\Xi_{0} c^{3} n_{1} n_{2} n_{3}+\left(w_{3}^{2}+W_{1}^{2}\right)} \cdot\left[T_{1} T_{2} T_{3} \exp \left(-\frac{\alpha_{2} ?}{2}\right)\right]
$$

where $d$ is the effective nonl inear coefficient of the crystal; $W_{3}$ and $W_{1}$ are the spot sizes (defined as the 1/e radii) of the incident Gaussian fields at $\omega_{3}$ and $\omega_{1} ; \alpha_{2}$ is the power absorption coefficient of the crystal at $\omega_{2} ; T_{3}$ and $T_{1}$ are the transmissions of the entrance face of the crystal at $\omega_{3}$ and $\omega_{1}$; and $T_{2}$ is the transmission of the exit face at $w_{2}$.

The most important feature evident in Eq. (9) is the scaling of the output power $P_{2}$ with the product of the input powers, $P_{1} \times P_{3}$, and the square of the crystal length, $\ell^{2}$. Another interesting feature is the quadratic dependence on the output frequency $w_{2}$. Even if all other parameters are constant, the factor $\omega_{2}^{-2}$ can result in a significant decrease in the power generated as the output wavelength $\lambda_{2}=$ $2 \pi c / \omega_{2}$ becomes large.

Another important parameter in the mixing process is the linewidth of the output radiation. Equation (9) applies to the phase-matched interaction of two monochromatic input beams. In this instance, the output radiation is monochromatic as well. This result must be modified to account for the finite linewidths of the input beams. The linewidth of the field generated at $\omega_{z}$ will scale with that of the broader of the two input fields at $\omega_{3}$ and $w_{1}$. If one or both of the input fields 
exceed the bandwidth over which phase-matching is maintained, then the linewidth of the output field at $\omega_{2}$ will be determined by the phasematching bandwidth. This, in turn, depends upon the crystal and the phase-matching technique employed in the mixing process.

\subsection{Previous Research}

Several reviews of the tunable infrared sources under development are available, including those of Kuhl and Schmidt ${ }^{2}$, Kelley', and most recently Mooradian ${ }^{4}$. The most promising primary sources are semiconductor diode lasers, F-center lasers and high-pressure gas lasers. Other sources of tunable infrared radiation, besides difference-frequency systems, include optical parametric oscillators, four-wave mixing systems, and stimulated Raman scattering systems. Within the Raman scattering category are those systems that employ fixed-frequency pump lasers and a tunable Raman shift such as the spin-flip Raman laser and the polaritron laser. A second group of Raman scattering systems achieve tunability by employing a variable-frequency pump laser (typically a dye laser).

In research to date, difference-frequency systems have achieved significantly broader wavelength tuning ranges than any of the primary sources being developed for tunable infrared operation. The primary limitation to the tuning range of a difference-frequency system is typically the transmission range of the mixing crystal. Differencefrequency systems require much lower input power levels than do fourwave mixing systems due to the relative magnitudes of the relevant 
nonlinear coefficients $\left(x^{(2)} \gg x^{(3)}\right)$. Optical parametric oscillators (which may be considered a special case of difference-frequency generation where the inpui field at $\omega_{1}$ is generated by noise in a cavity resonant at $\left.\omega_{2}\right)$ are difficult to $l$ ine narrow. Given the combined requirements of wide tunability, narrow linewidth and high average power in a relatively simple system, difference-frequency generation is an attractive means of producing tunable infrared radiation.

Difference frequency generation of tunable infrared radiation was first demonstrated by Dewey and Hocker in $1971^{5}$. By mixing the outputs of a ruby laser $\left(\omega_{3}\right)$ and a tunable dye laser $\left(\omega_{1}\right)$ in a phasematched lithium niobate $\left(\mathrm{LiNbO}_{3}\right)$ crystal they succeeded in generating relatively high peak power (10 KW) radiation in the 3-4 um spectral region. Synchronization of the two iaser pulses was insured by pumping the dye laser with part of the output of the ruby laser. Subsequent research has explored variations of this basic system. Ruby and dye lasers have been mixed in lithium iodate $\left(\mathrm{LiO}_{3}\right)$ where axial pumping and dichroic mirrors were employed in the dye laser to allow the crystal to be placed within the laser cavity for enhanced efficiency ${ }^{6}$. This configuration produced $100 \mathrm{~W}$ peak power in the 4.1-5.2 $\mu \mathrm{m}$ spectral region. In similar work ${ }^{7}$, a frequency-doubled Nd: YAG laser was substituted for the ruby pump to produce 3.4-5.65 im infrared output at pulse repetition frequencies of up to $80 \mathrm{~Hz}$ and peak power of $.5 \mathrm{~W}$. Extracavity mixing has also been performed using frequency-doubled $\mathrm{Nd}$ : YAG and dye lasers 8,9 . In one case ${ }^{8}, 2.8-3.4$ um radiation was generated at up to $80 \mathrm{~mW}$ peak power. 
In each of the above experiments, a narrow-band pump (ruby or Nd:YAG laser) has been mixed with a tunable, relatively broad-band dye laser, with the latter determining the infrared output linewidth. In an effort to achieve wide tunability, several authors have reported mixing two tunable dye lasers. In one arrangement, two dye lasers, pumped by the same ruby laser, were mixed in a proustite $\left(\mathrm{Ag}_{3} \mathrm{AsS}_{3}\right)$ crystal to produce infrared radiation in the $3.20-5.65 \mathrm{\mu m}^{10}$ and $5.82-$ $7.25 \mathrm{um}^{11}$ regions at peak powers ranging fron the $1 \mathrm{KW}$ to $100 \mathrm{~W}$ range. The authors reported some loss of efficiency due to the difficulty of phase-matching two relatively broad-band inputs.

Proustite has been used in several experiments because of its broad transmission range ${ }^{3,10-12}$. In one investigation, two ruby-pumped dye lasers were mixed to produce $11-23$ um radiation at up to $3 \mathrm{~W}$ peak power $^{12}$. Two dye lasers pumped by a nitrogen laser were mixed in a crystal of silver gallium sulfide $\left(\mathrm{AgGaS}_{2}\right)$ to generate up to $4 \mathrm{~W}$ peak power from $5.5-18.3 \mathrm{\mu m}^{13}$.

Gerlach $^{14}$ employed two dye lasers and a lithium iodate crysta? to produce the most complete coverage of the $2-5 \mathrm{jm}$ region reported to date. By pumping with two synchronized flashlamps he produced up to $.4 \mathrm{~W}$ peak power from 1.5-4.8 um.

Two continuous-wave $(\mathrm{cw})$ systems have been reported ${ }^{15,16}$. Both operated at microwatt power levels below $5 \mathrm{um}$ in wavelength. Pine's 15 apparatus was tunable from 2.2-4.2 um with a linewidth of less than $15 \mathrm{iHz}\left(5 \times 10^{-4} \mathrm{~cm}^{-1}\right)$. Although capable of narrower linewidth generation, cw mixing suffers from extremely low conversion efficiency due to the low power levels of the mixing sources. 
Several authors have pumped optical parametric oscillators using either the pump and signal waves ${ }^{17}$ or the signal and idler waves $18-20$ for mixing in a variety of crystals. Silver gallium selenide $\left(\mathrm{AgGaSe}_{2}\right)^{17}$, cadmium selenide $(\mathrm{CdSe})^{18,19}$ and gallium selenide (GaSe) $)^{20}$ have been employed in experiments that have generated infrared radiation in spectral regions between 4 um and $24.3 \mathrm{jm}$.

\subsection{Configuration of the Present System}

The difference-frequency generation system investigated here is similar in form to other systems (Figure 1). A relatively narrowlinewidth pulsed pump laser $\omega_{3}$ is mixed with a tunable dye laser $w_{1}$ in a nonlinear crystal $\left(\mathrm{LiNbO}_{3}\right)$. A portion of the pump beam is used to pump the dye laser. This insures pulse synchrorization.

In this case the pump laser is a lead vapor laser (LVL). It operates at a wavelength of $\lambda_{3}=.7229 \mathrm{\mu m}$. As with other metal vapor lasers, the LVL can be run at a very high pulse repetition frequency (PRF). It has been operated here at $2 \mathrm{KHz}$ while elsewhere a similar laser has been run at five times this rate ${ }^{21}$. In contrast, $80 \mathrm{~Hz}$ is the highest PRF reported in other difference-frequency systems ${ }^{7}$ and most have been restricted by the pump laser to much lower rates. The result is potentialiy a far higher ratio of average power to peak power in the present system than in previous systems.

The tunable dye laser is constructed with very broad band optics that permit operation in the $.72-1.15$ im spectral region. Pumped by the LVL, it could be operated from approximately .80-1.01 um using 


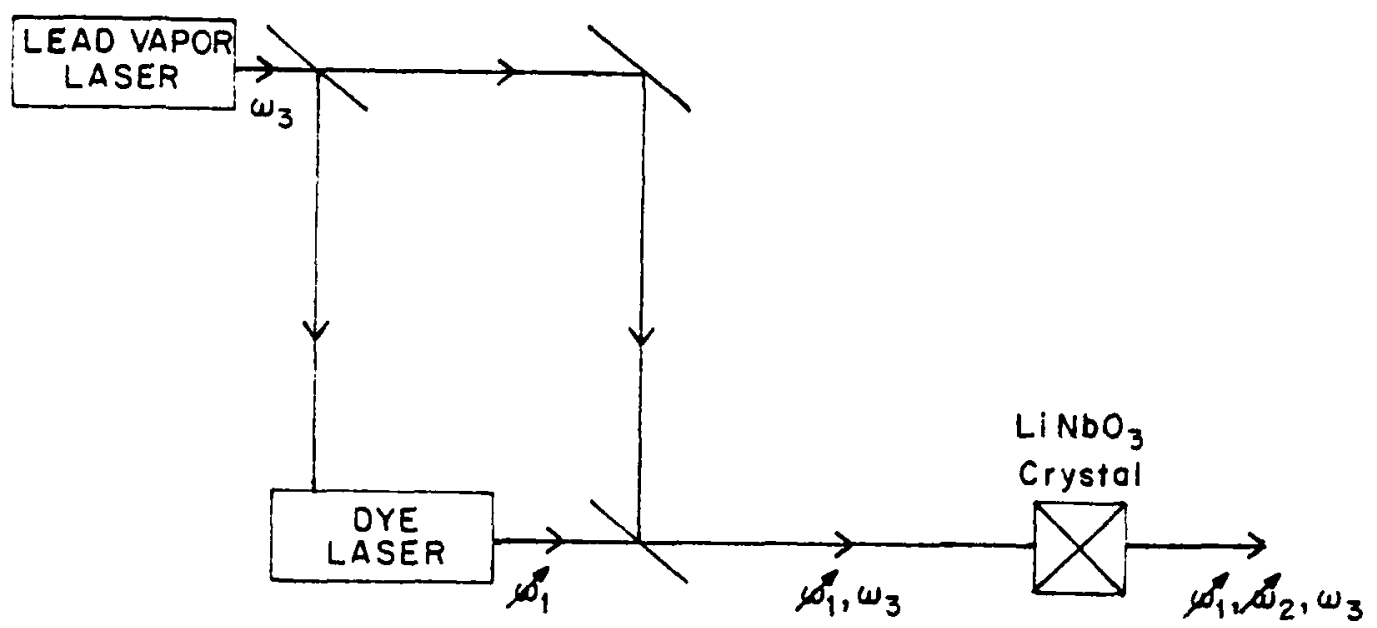

Figure 1. Difference-frequency generation system. 
several dyes. Difference-frequency mixing across this range would result in the following infrared wavelengths (in im):

\begin{tabular}{|c|c|c|}
\hline$\lambda_{3}$ & $\lambda_{1}$ & $\lambda_{2}$ \\
\hline .7229 & .80 & 7.50 \\
\hline .7229 & .85 & 4.83 \\
\hline .7229 & .90 & 3.67 \\
\hline .7229 & .95 & 3.02 \\
\hline .7229 & 1.00 & 2.61 \\
\hline
\end{tabular}

Absorption by the lithium niobate crystal limits the infrared generation to wavelengths below approximately $4.5 \mathrm{um}$. Therefore the operational tuning range of this systein will be approximately

$$
2.5 \mu \mathrm{m}<\lambda_{2}<4.5 ; \mathrm{m}
$$

Conceivably a crystal such as proustite could be substituted to extend this range further into the infrared. The present systern is limited to wavelengths greater than 2.5 um because dyes that lase beyond about 1.1 um are not available.

Phase-riatching is achieved in this system by controlling the temperature of the lithium niobate crystal in an oven. For the wavelengths involved, the crystal temperature must be varied in the $450^{\circ}$ $550^{\circ} \mathrm{C}$ range. The LVL and the dye laser must be of opposite polarizations at the crystal in order to satisfy the phase-matching requirements. 


\section{THE LEAD VAPOR LASER}

This Chapter describes the lead vapor laser and the associated discharge circuitry. The final Section of this chapter presents experimental results concerning the efficiency, lifetime, and mode quality of this laser.

\subsection{Laser Emission at .7229 $\mathrm{um}$}

Metal vapor lasers that operate on self-terminating transitions are attractive for infrared difference-frequency generation because of two characteristics. The first is that they are quite efficient. The second is that they are capable of operation at pulse repetition frequencies of several kilohertz and, therefore, they can produce high average power even though the pulse energy is smali.

Among this group, the lead vapor laser has distinct advantages for use in the present mixing system. Ooerating on the red 1 ine at $\lambda=$ $.7229 \mathrm{um}$, it can directly pump an infrared dye laser. In addition, the operating temperature of the LVL $\left(\cong 1050^{\circ} \mathrm{C}\right)$ is substantially lower than that of other metal vapor lasers. This simplifies the construction and operation of the laser by permitting the use of a quartz discharge tube instead of alumina tubes (as required, for example, in copper vapor lasers).

A potential energy level diagram for lead 21,22 is shown in Fig. 2. The red line at $.7229 \mathrm{um}$ operates on the $\left(7 s^{3} P_{1}-6 p^{2}: D_{2}\right)$ transition. The upper laser level is populated by electronic excitation during a 


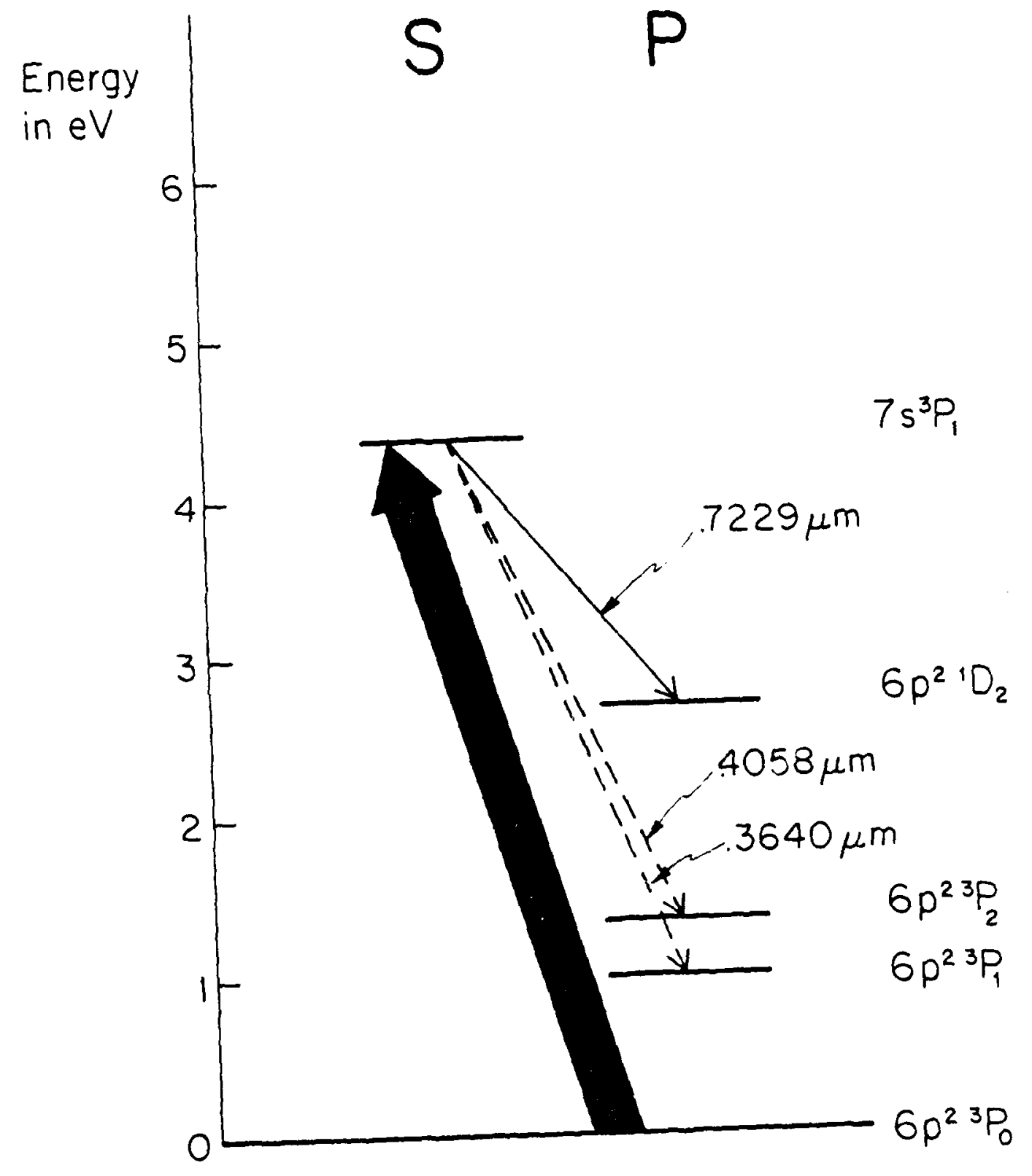

Figure 2. Partial energy ?evel diagram of $P b$. 
fist-rising longitudinal current pulse. The lower level is a metastable state that is depopulated primarlly by collisions at the tube wall. Two other lines at $.3640 \mathrm{um}$ and $.4058 \mathrm{um}$, share the upper state of the red line and compete with it. However, as the vapor temperature increases, the terminal levels of the $.3640 \mathrm{um}$ and $.4058 \mathrm{um}$ lines become themally populated to the extent that emission on these lines is suppressed 23 . Calculation indicates that transitions on both the .3640 im and .4058 im lines are innibited by resonance trapping at temperatures above $900^{\circ} \mathrm{C}$. The temperature required to suppress the satellite lines is well below that at which the terminal level of the red line has a significant thermal population. For this reason the LVL can be operated so that virtually all of the emission occurs at $.7229: \mathrm{m}$. Feldman et a: ${ }^{21}$ have demonstrated the strong temperature dependence of the LVL output pulse energy at $.7229 \mu \mathrm{m}$. The peak temperature occurs at close to $1050^{\circ} \mathrm{C}$, and exhibits a small dependence on discharge conditions and tube geometry; the output pulse energy is down by $25 \%$ at about $30-40^{\circ} \mathrm{C}$ from the maximum and continues to decline sharply as the temperature within the discharge tube deviates from the optimum temperature. The decline of the output pulse energy at temperatures above the peak is attributed to thermal population of the lower laser level.

\subsection{Description of the Lead Vapor Laser}

The present LVL operate; at a temperature of approximately $1050^{\circ} \mathrm{C}$. It is initially filled with buffer gas, either helium or neon, at a pressure of about 4 Torr. This pressure is close to the vapor pressure of lead ( $\approx 3$ Torr) at $1050^{\circ} \mathrm{C}$. 
The basic LVL component is a $48^{\prime \prime}$ long quartz tube, the central section of which is heated to operating temperature in a furnace. The furnace consists of a 42 " long mullite tube with a l" inside diameter around which are coiled 122 turns of 14 gauge nichrome wire (total cold resistance $\cong 12 \ldots$ ). This assembly is wrapoed by 2 " of Kacwool blanket thermal insulating material. Approximately twenty minutes are required to heat the cold discharge tube to operational temperature. The quartz tube is supported at each end by modified brass vacuum fittings which also serve as electrodes. Copoer tubes 3 " in length with inside diameters of $3 / 4^{\prime \prime}$ are soldered to the fittings and are cut at Brewster's angle to the laser axis. Glass windows are epoxied to the tube ends. Both electrodes are fitted with vacuum ports, al though the high yoltage (cathode) port is tvpically sealed off while the ancde port is connected to the burfer gas manifold. Several turns of $1 / 4^{\prime \prime}$ copper tubing are soldered to the electrodes to provide for water cooling. This protects the 0-rings of the vacuum fittings and the window epoxy from the combined effects of the onmic neating of the electrodes and the thermal conduction of the quartz tube. The entire assembly is shown in Fig. 3 .

The discharge tube is supported on a lathe bed optical rail. The output coupler and rear reflector are mounted kinematically on the rail as well. The laser can be rapidly disassembled for cleaning and recharging.

Two alternative LVL designs have been demonstrated elsewhere. Feldman $2 \pm x:{ }^{21}$ have run an all-hot LVL in which the discharge tube 


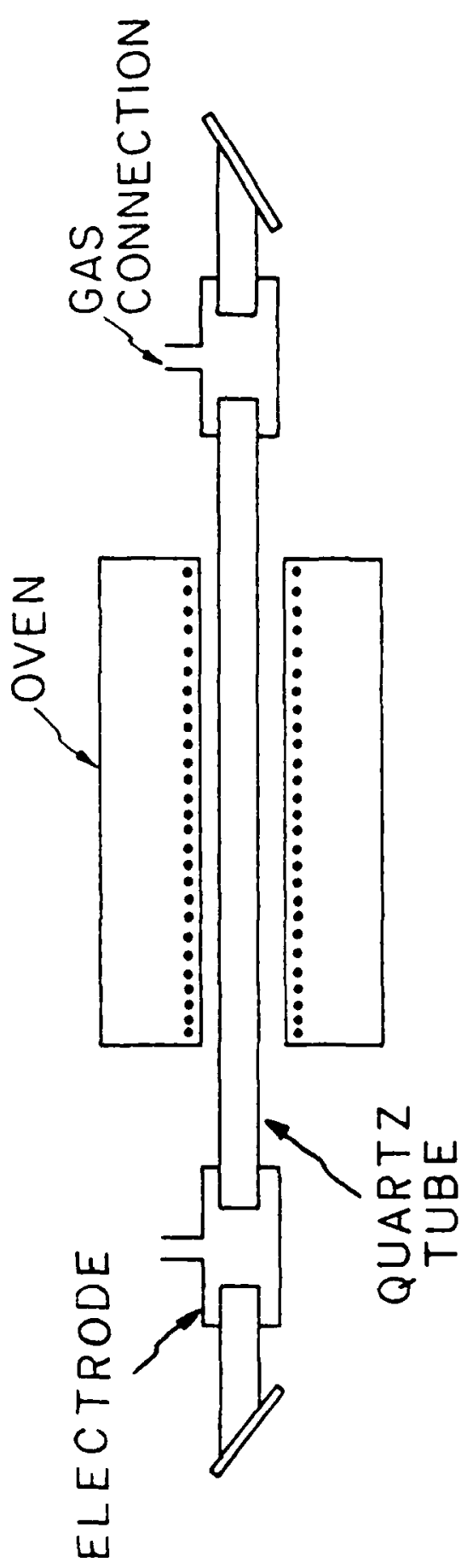

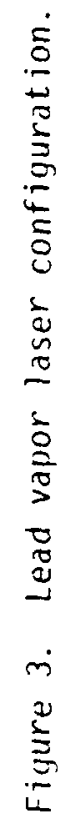


was sealed off; quartz-molybdenum electrical feedthroughs were used, and the entire laser tube was placed within an oven. Anderson et $a: 24$ have operated a system employing a continuous gas flow and cold electrodes. However, they operated without a furnace in a discharge-heated mode with a well-insulated discharge tube.

Before use, the present laser tube is evacuated to a pressure of 5 mTorr at $400^{\circ} \mathrm{C}$. This prevents the formation of lead oxide which, at operating temperature, reacts with quartz. The vacuum pump is isolated from the tube by a molecular sieve trap.

\subsection{The Pulse-Forming Circuit}

Lead atoms are excited by electron collisions to the $7 \mathrm{~s}^{3} \mathrm{p}$ : revel ( $4.36 \mathrm{eV}$ above the ground state) during each longitudinal current oulse. The pulse-forming circuit is showr in Fig. 4. An E. G. \& G. Model 1802 Hydrogen Thyratron acts as a fast switch to initiate the pulse after the capacitor has charged up to twice the suppiy voltage.

After each discharge pulse, the thyratron turns off and the capacitor charges through the indicator. This inductive charging occurs in a time $T_{L C}=\pi \sqrt{L C}=.44 \mathrm{~ms}$. This is much longer than the time constant of the resistive charging, $\tau_{R C}=R C=.02 \mathrm{~ms}$, associated with the $10 \mathrm{~K}$ : resistor. In effect, the $10 \mathrm{Ka}$ resistor acts like a short circuit- to ground during the inductive charging cycle of the capacitor. The resistor must be large compared to the impedance of the discharge tube during breakdown, however, so that significant pulse energy is not shunted around the tube. 


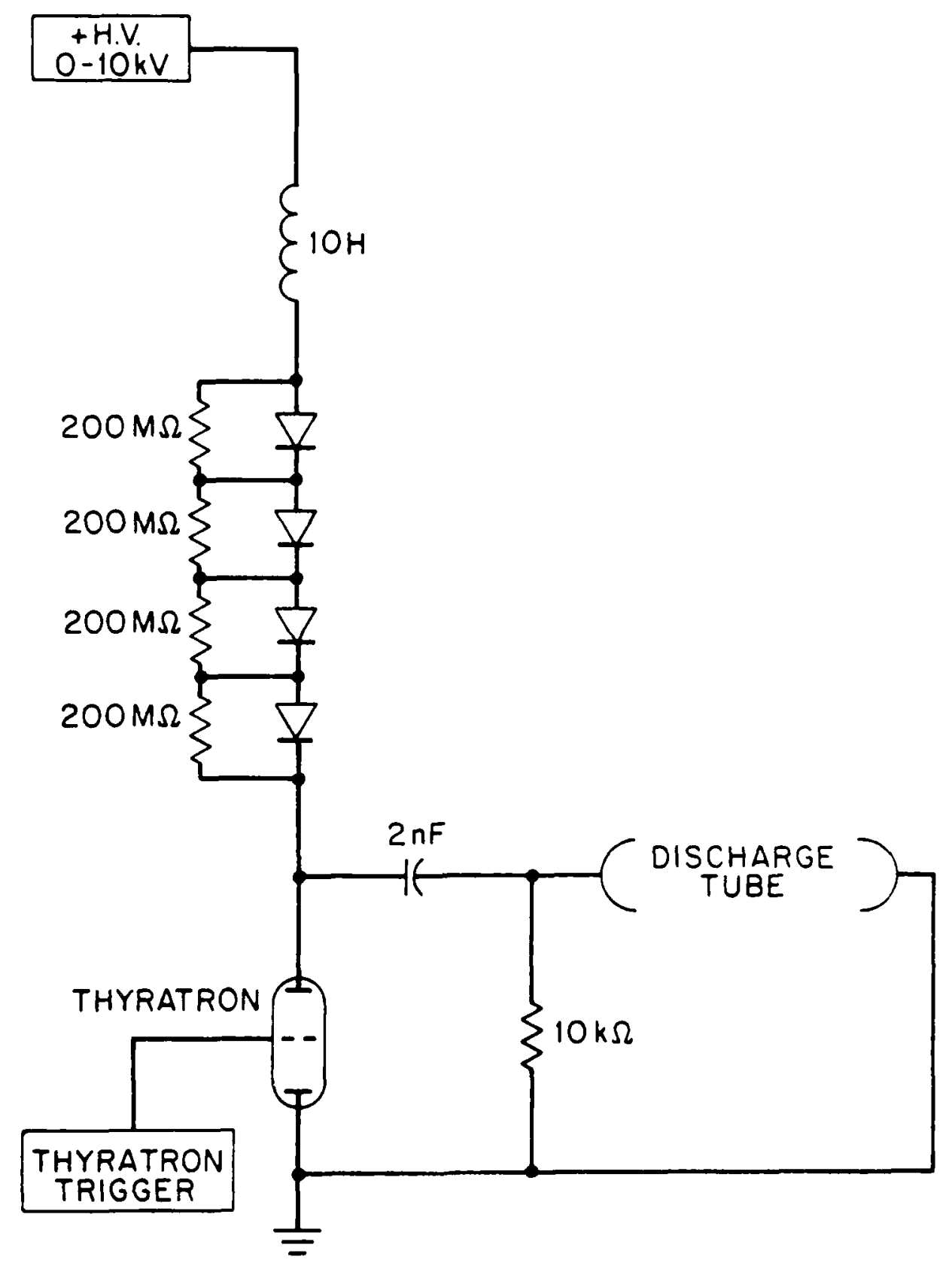

Figure 4. Lead vapor laser discharge circuit. 
The charging of the capacitor at the anode of the thyratron to tivice the supply voltage, $V_{s}$, is explained as follows: with the capacitor fully discharged, the voltage across the inductor, $V_{L}=$ $\mathrm{Ldi} / \mathrm{dt}$, is equal to the supply voltage; the rate of change of the current, $d i / d t$, is maximum when the charging current begins to flow. When the capacitor voltage, $V_{C}$, has charged up to the supply voltage, $V_{L}=0$ and the rate of change of the current has decreased from its maximum value to zero. This is the point at which the maximum charging current is achieved. The rate of change of the current then decreases to its minimum (i.e. most negative) value at which point $V_{L}=-V_{S}$. The charging current is zero here and $V_{C}=2 \cdot V_{s}$.

The diodes hold this voltage by preventing current reversal. They are each rated at $10 \mathrm{KV}$ and are protected by $200 \mathrm{M} \Omega$ resistors. These resistors insure that each diode drops an equal portion of the held-off voltage $\left(V_{s}\right)$. The time required for the capacitor to charge to full voltage is $T_{L C}$. Because this charging time is much greater than the pulse time, it determines the maximum PRF allowed for circuit operation:

$$
P R F_{\max }=T_{L C}^{-1}=2.3 \mathrm{KHz}
$$

When the thyratron is triggered, it conducts and pulls its anode voltage to ground, thus developing a voltage equal to $2 \cdot v_{s}$ across the discharge tube. When the tube breaks down, a large current pulse occurs as the capacitor discharges. During the pulse, the circuit in series with the capacitor (including the thyratron, discharge tube and 
conductors) has sufficient inductance to cause the voltage at the anode of the thyratron to ring. The anode voltage becomes sufficiently negative to turn off the thyratron and initiate the inductive charging cycle of the capacitor.

The full width at half the maximum amplitude of a typical current pulse is 100-150 ns. The risetime is 50-75 ns. During the pulse, the peak current exceeds 200 amperes. In Fig. 5 typical current and voltage waveforms are shown. The current pulse displayed in the upper trace was measured with a Tektronix Model P-6021 Current Probe and Model CT-5 High Current Transformer at the laser anode. The probe has a measured time delay of $2 \mathrm{~ns}$. The cathode voltage appears on the lower trace. A Tektronix Model P-6015 1000x Voltage Probe with a time delay of less than 1 ns was used. The high frequency ringing that appears on both traces of the photograph is due to the CT-5 transformer.

\subsection{Experimental Results}

The development of the LVL has centered on the optimization of three characteristics: efficiency, lifetime and mode quality. In addition, a peak pulse energy of at least $100 \mu \mathrm{J}$ has been considered necessary to perform preliminary difference-frequency mixing experiments. Refinement of the LVL is made difficult by the interdependence of such parameters as vapor temperature, buffer gas pressure, PRF and capacitor voltage. It is not possible to isolate their effects on the output pulse energy and width. In addition, the discharge is not understood well enough to determine a propi the optimum conditions within the tube 


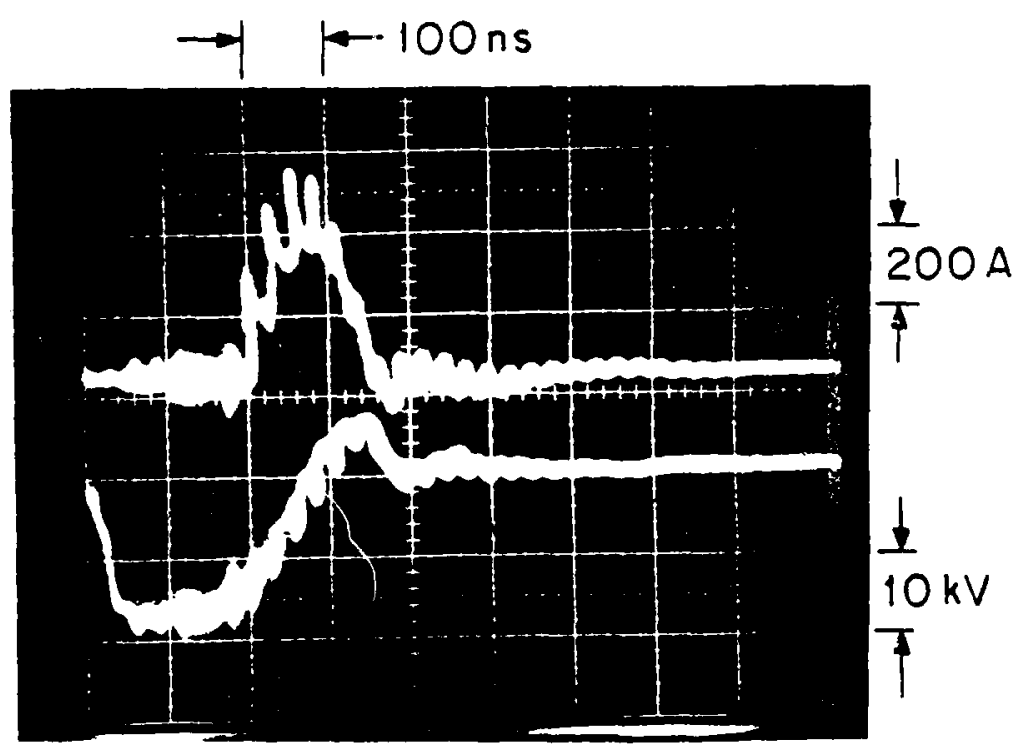

Figure 5. Lead vapor laser current (upper) and voltage (lower) waveforms. 
or the best electrode design. A number of electrodes, charging circuits, tube designs, buffer gases, operating conditions and other component adjustments have been examined during the course of this research. In this Section, the LVL is characterized as it now stands while some consideration is given to certain design alternatives that have been studied.

The most serious problem encountered in the development of the LVL is the diffusion of lead out of the hot region of the discharge tube to the cold ends. In a straight tube, as described in Section 3.2, Iiquid beads of lead form at the bottom of each end of the tube. These beads decrease efficiency and degrade mode quality because they aperture the laser. Diffusion 1 imits the lifetime of the laser because all lead is eventually removed from the active volume.

A possible solution to this problem, short of going to an all-hot design, is the use of a heat pipe 25,26 to return the liquid lead to the hot region of the tube. Wicks consisting of rolled wire mesh are placed within the tube, bridging the cold and hot regions. Idealiy a steady state is established in which liquid lead is returned to the hot region through capillary action in the wicks. The heat pipe requires a wick material that is wetted by lead and that withstands the LVL operating temperature. Nickel, molybdenum and stainless steel were considered as potential wick materials. Wicks as fine as 100 mesh were tested in the LVL. The nickel wicks showed some tendency to wet but softened during use and collapsed, blocking the tube. The molybdenum did not wet even when great care was taken to clean the material before use. Burnham and $0 j e u^{27}$ have reported a lead vapor heat pipe using stainless steel mesh. However, the ir result could not be reproduced here. 
A second solution to the lead migration problem is currently in use in the LVL. The solution is to use a "barbeil" discharge tube (Fig. 6). This tube consists of five quartz sections that are fused together. The long central section and the two end sections which fit into the electrode vacuum fittings are of one diameter. The other two sections that extend through the short regions in which a steep thermal gradient exists have a larger diameter. Lead beads form in the large diameter sections where they do not aperture the laser. With a sufficient amount of lead, the beads extend far enough into the hot region to return lead that has diffused away. As in the heat pipe, a steady state circulation is established that allows the LVL to be operated essentially indefinitely.

The barbell tube has inside diameters of $16 \mathrm{~mm}$ and $22 \mathrm{~mm}$. The large diameter sections are $5^{\prime \prime}$ long and are $2^{\prime \prime}$ from the tube ends. The overall tube length is $48^{\prime \prime}$.

Neon is chosen as the buffer gas over helium because slightly higher output pulse energy is achieved with it. During laser operation, the buffer gas pressure is maintained at approximately 4 Torr in the tube, al though varying the pressure from 3-5 Torr has little effect on the output pulse energy. At higher pressure, the output pulse energy decreases because the time required to break down the tube increases and the current pulse slows. At lower pressures, the discharge typically becomes unstable.

The barbell tube LVL has consistently operated at a puise energy of $125 \mathrm{uJ}$, a $30 \mathrm{~ns}$ pulsewidth, and a polarization ratio close to $20: 1$. 


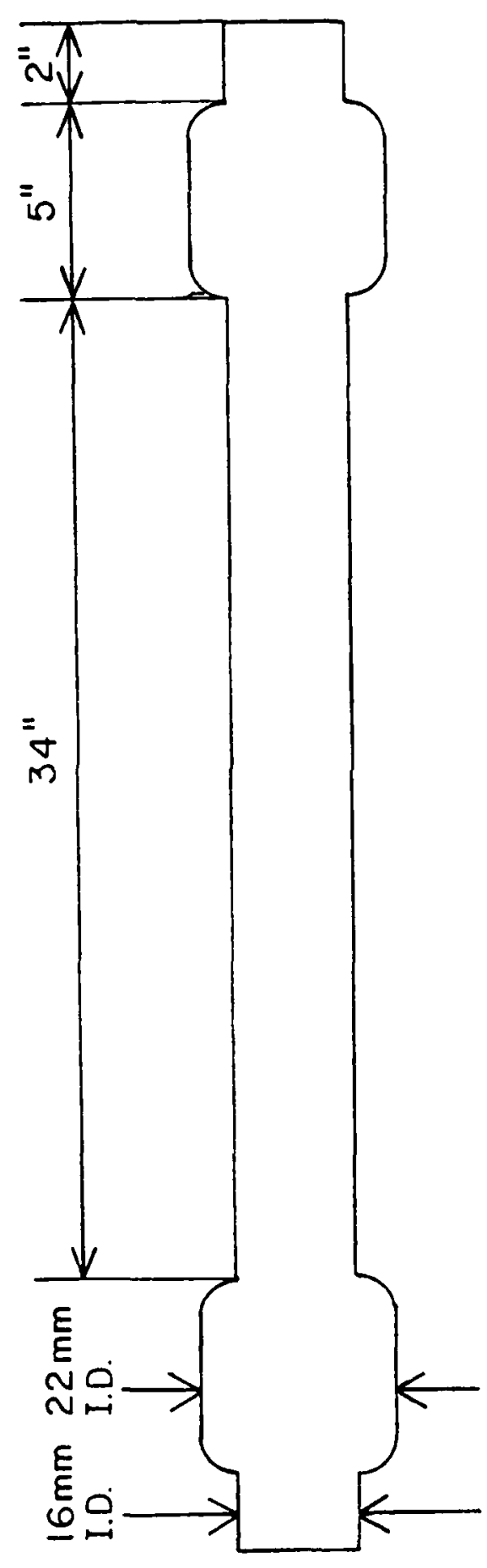

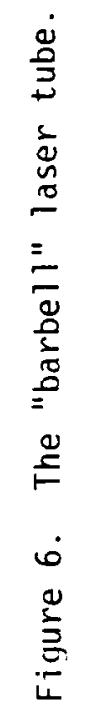


Energy measurements were made with a Molectron Model J3 Pyroelectric Detector and the pulsewidth was measured with a Tropel Silicon PIN Photodiode (total system risetime $<2 \mathrm{~ns}$ ). The tube does not require recharging after more than 25 hours of operation. Neither the lifetime nor the mode quality are limited by lead diffusion within the tube. The active tube length is approximately $90 \mathrm{~cm}$ so that the peak specific output pulse energy was $.69 \mathrm{jJ} / \mathrm{cm}^{3}$. The laser was operated with a $2 \mathrm{nF}$ capacitor that was charged up to approximately $18 \mathrm{KV}$ before breakdown. The peak energy efficiency was .039\%.

The highest pulse energy and efficiency reported for a LIL is that of Feldman e $=a 2^{21}$. In a $1.2 \times 100 \mathrm{~cm}$ tube, they produced a peak output pulse energy of $230 \ldots \mathrm{J}$, a specific pulse energy of $2.0 \mathrm{uJ}$ ' $\mathrm{cm}^{3}$ and a peak efficiency of . $12 \%$.

The LVL efficiency is apparently limited here by the relatively long risetime of the current puise. Presumably a faster-rising pulse would result in a greater peak population inversion and higher peak pulse energy. In Fig. 7 a typical current pulse is shown along with the laser output pulse. The ringing on both traces is due to the CT-5 transformer. It is evident that the laser pulse occurs well in advance of the current peak. Fig. 8 shows the cathode voltage and the laser pulse. The very high frequency "hash" evident in the photograph is electromagnetic interference that is radiated by the discharge circuit.

In order to speed up the current pulse, the inductance in series with the capacitor was minimized. The pulsewidth, proportional to the square root of the series inductance, indicates a total inductance on 


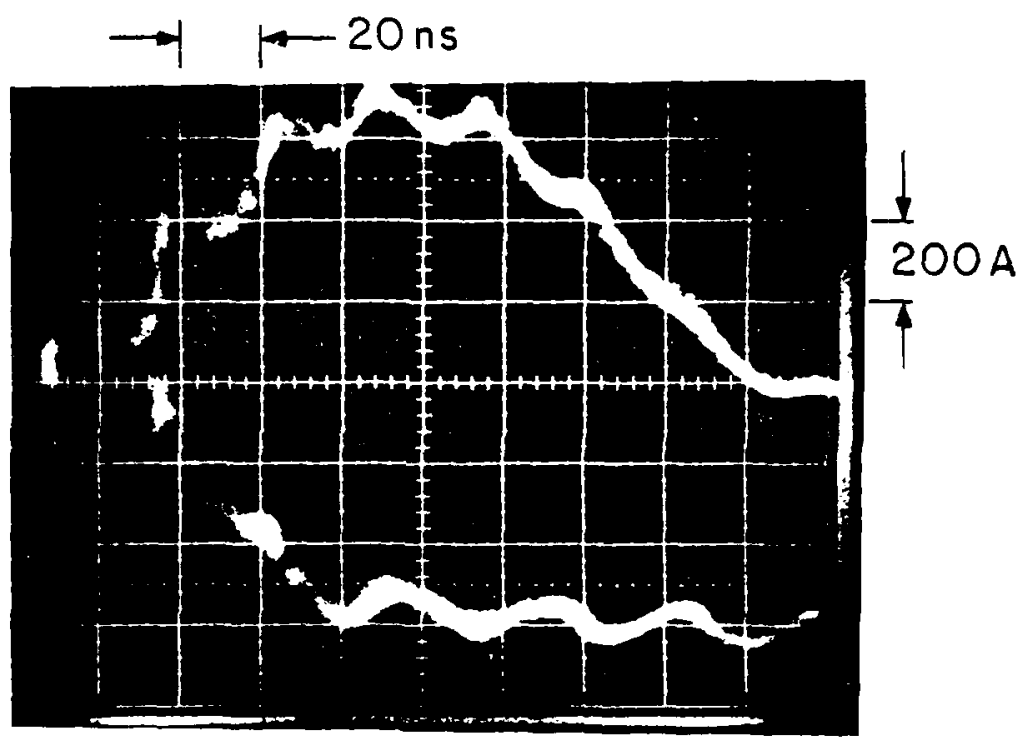

Figure 7. Lead vapor laser current (upper) and output (lower) waveforms. 


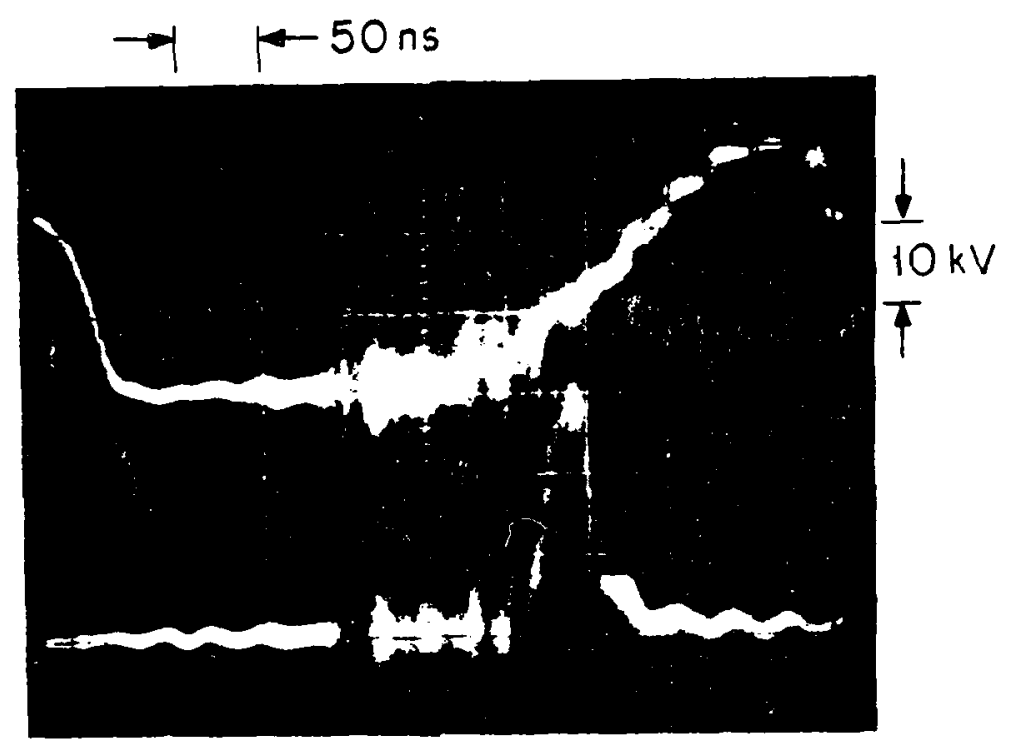

Figure 3. Lead vapor laser voltage (upper) and output (lower) waveforms. 
the order of $500 \mathrm{nH}$. The thyratron inductance is about $20 \mathrm{nH}$ while that of the two Condenser Products Corporation $1 \mathrm{nF}$ capacitors is 20-40 $\mathrm{nH}$ each. This indicates that the bulk of the circuit inductance is distributed over the length of the discharge tube and the circuit conductors (copper strips approximately $.01 " \times 2 "$ ). It is interesting to note that the higher-efficiency all-hot laser demonstrated by Feldman zt $x_{2}, 21$ had three electrodes. The cathode was at the center of the discharge tube and e two anodes were at the ends 28 . Modeling the discharge as a distributed inductance, it is evident that this electrode arrangement resulted in one quarter of the discharge inductance of a similar tube with the cathode and anode at opposite ends.

In order to reduce the total inductance the discharge circuit was rebuilt in a coaxial configuration. A stainless steel tube was placed within the furnace around the discharge tube. It vas threaded into the anode vacuum fitting and connected at the other end to copper sheets that surrounded the charging capacitors and bolted to the cathode of the thyratron. All conductor lengths were minimized as the circuit components and the discharge tube were maintained in close proximity. Despite these improvements, no pulse shortening or increase in output pulse energy was observed.

The size of the capacitor was experimentally chosen. While the risetime of the current pulse is proportional to the square root of the capacitance, the energy of the discharge is directly proportional to it. Capacitors of $1 \mathrm{nF}, 2 \mathrm{nF}$ and $5 \mathrm{nF}$ were tested. The greatest output energy was observed with the $2 \mathrm{nF}$ capacitor. 
The uniformity of the discharge also critically affects the efficiency of the LVL as well as the output mode quality. Filamentary discharge behaviour has been observed with some electrodes resulting in the incomplete excitation of the lead vapor and an irregularlyshaped beam.

As the PRF is increased to pulse rates approaching $2 \mathrm{KHz}$ the time required for the tube to break down is drastically reduced. The tube begins to conduct before the voltage across it peaks $(\approx 20 \mathrm{nsec}$ after the thyratron fires). This is attributed to residual ionization of the cold helium buffer gas between the electrodes and the lead vapor. In addition, self-heating occurs within the discharge tube at high PRF. Feldman ei $a i .^{2 l}$ suggest that this may affect efficiency at lower operating temperatures by inducing a radial thermal gradient within the tube that results in the depletion of the lead density aiong the discharge axis. Evidence of this was seen by Feldman $2 \div x_{2} .^{21}$ and we have confirmed this result when operating the laser between $950^{\circ} \mathrm{C}$ and $1000^{\circ} \mathrm{C}$ in a "burst mode" where initial output puises are observed to be of higher energy than subsequent ones.

The above results were achieved with a maximum reflectivity, flat rear reflector and a $35 \%$ reflective, flat output coupler. Higher output pulse energy is obtained with lower reflectivity output couplers. However the increased efficiency is accompanied by a degradation of the output mode quality. The LVL has been operated at high pulse energies with the output coupler removed. The resulting pulse is quite difficult to focus. The mode quality is very sensitive to any light 
that is coupled back into the laser cavity. With low output reflectivities $(5-10 \%)$ the mode is considerably improved and can be focussed tightly enough to pump the dye laser. However, for use in the mixing experiment the LVL beam must be focussed to a spot size on the order of $100 \mathrm{jm}$ over a distance of $1 \mathrm{~cm}$. This requires even better mode quality and therefore a higher output reflectivity. The $35 \%$ reflective output coupler was found to be the best compromise between efficiency and mode quality. The LVL beam can be focussed to a circular spot that passes cleanly through a $100 \mathrm{~mm}$ slit. The LVL was also tested with a 7 m radius of curvature spherical rear reflector. The stable cavity resulted in slightly less efficiency and no appreciable improvement in the mode quality. 


\section{THE DYE LASER}

In this Chapter the dye laser design is described in detail. The final Section presents experimental results obtained with this 1aser.

\subsection{Basic Configuration}

The foremost dye laser design requirement is that the laser operate substantially over threshold with the available excitation pulse from the LVL of about $100 \mathrm{~J}$ of energy and $30 \mathrm{~ns}$ pulsewidth. This is an extremely low pump pulse energy for the operation of a dye laser.

Two basic design considerations result directly from these pump limitations. First, the cavity mode at the dye cell must have a very small cross-section. This is to permit all of the dye volume that is intersected by the internal cavity mode to be excited above the threshold intensity level. In addition, the cavity length L must be small enough that the round trip time $2 \mathrm{~L} / \mathrm{C}$ is less than the pump pulsewidth. In general, the dye laser efficiency will increase and the linewidth will decrease as the ratio of pump pulsewidth to cavity round trip time increases. (In the limit, as the ratio approaches infinity, $\mathrm{cw}$ operation is approached.)

Two other design considerations are the requirements of wavelength tuning and line narrowing. A dispersive element such as a prism or a diffraction grating is requin $-r$ wavelength tuning. The resolution that is achieved with both the prisi.. and the grating increases with the 
diameter of the incident beam. Therefore, a relatively large cavity mode is required at the dispersive element to enhance selectivity. Line narrowing can be further improved by placing one or more FabryPerot etalons within the cavity. For experimental purposes, it is desirable to design the dye laser so that etalons can be added or removed without significantly disturbing laser operation.

An attractive approach to meeting these design requirements is the "dänsch cavity" 29 in which the dye cell is positioned between the output mirror and two lenses which form a beam-expanding telescope. The expanded beam is incident on a Fabry-Perot etalon and a diffraction grating in Littrow mount. The telescope enhances the dispersion of the grating and significantly increases the efficiency of the dye laser by reducing diffraction losses.

Although this design seems to have all of the required characteristics, it also possesses one serious flaw: the dispersion of the lens material can disturb dye laser operation. Achromatic lenses in the near infrared $(0.8-1.1 \mathrm{jm})$ region of interest are normally unavailable. For this reason a "z-cavity" design was chosen; it functions in a manner similar to the Hänsch cavity ${ }^{29}$ but requires no internal lenses. The z-cavity (Figs. 9 and 10) employs two spherical mirrors at nearnormal incidence to provide an internal, non-dispersive telescope. The dye cell is placed at Brewster's angle in the output leg to minimize reflection loss and to serve as a polarization-selective element. It is pumped off axis by the focussed LVL beam. Dispersive optics are placed in the back leg of the cavity. The experiments reported here 


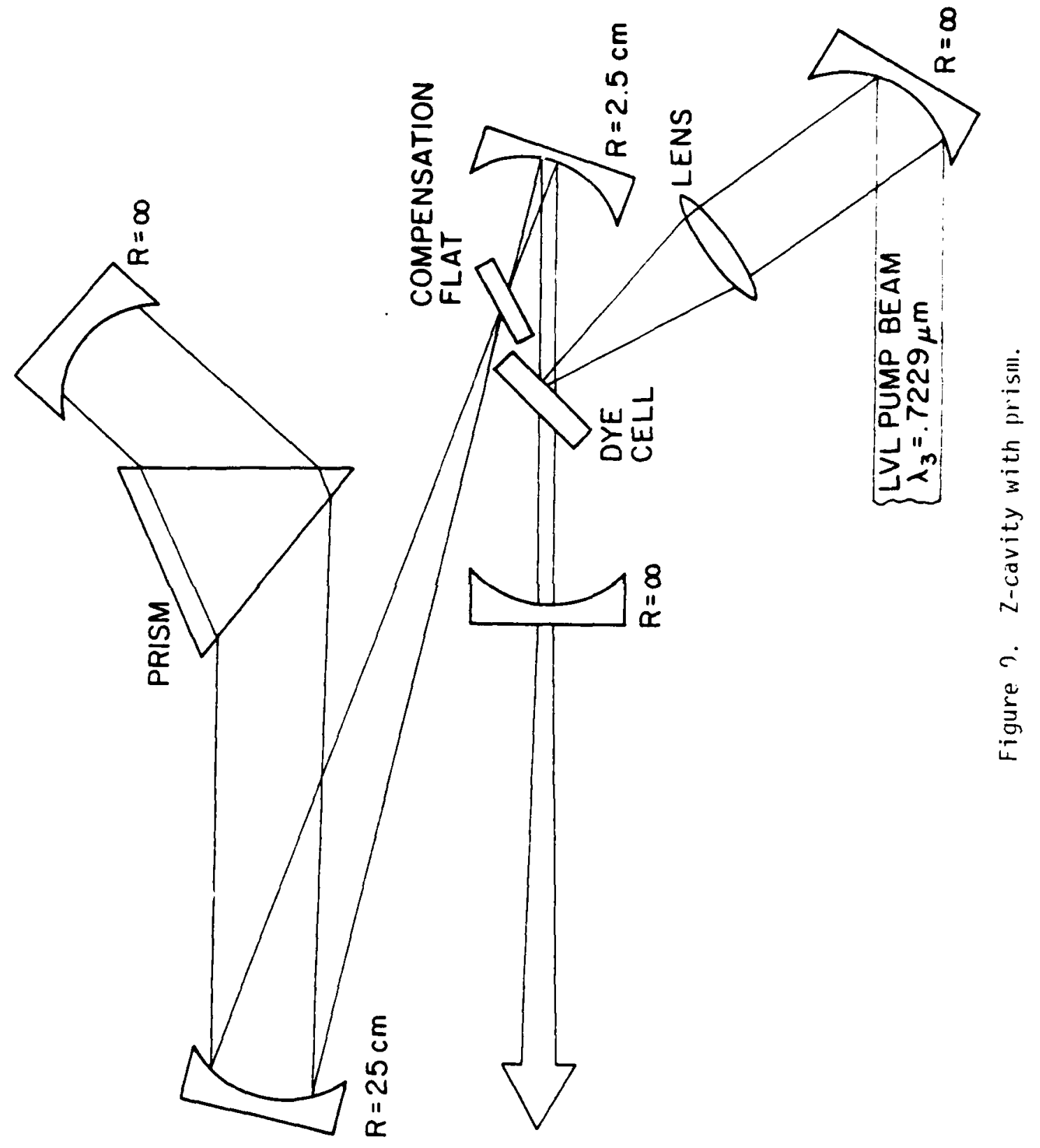




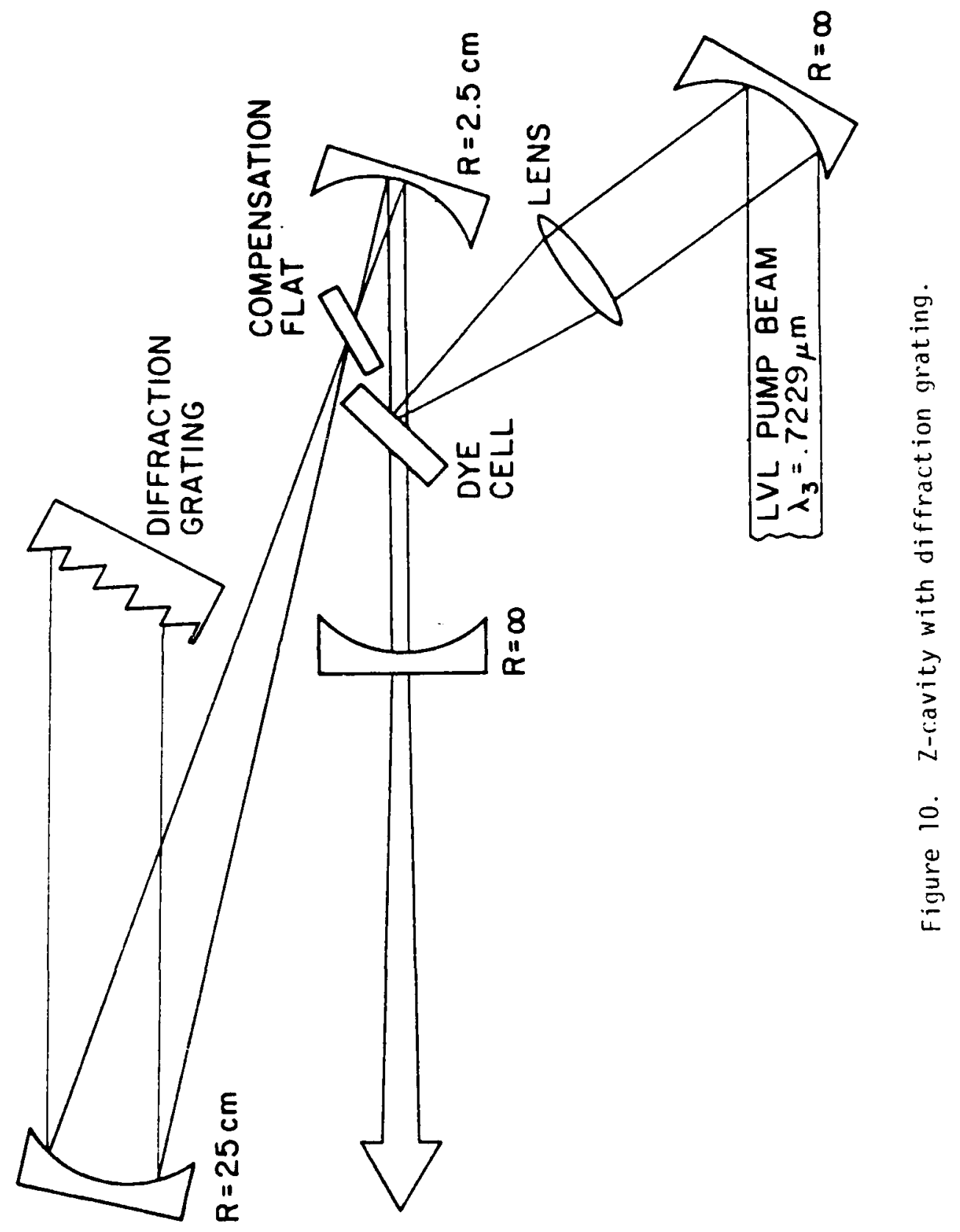


were performed using $10 \times$ beam expansion in conjunction with a $60^{\circ}$ prismflat mirror combination (Fig. 9). The prism was operated at minimum deviation, and the laser wavelength was tuned by rotating the rear mirror. A Brewster-angle prism is preferred in this design, but such a prism was not available during this research. An alternate method of line tuning that generally offers higher dispersion at a lower efficiency is to use a diffraction grating (Fig. 10) that can be rotated in a manner similar to that of the rear cavity mirror. This arrangement has also been demonstrated. One or more etalons can be placed in the rear leg for further line narrowing.

The dye laser cavity has been designed to have a high finesse, to allow many round trips per pump pulse, and to be stable. A high finesse cavity is necessary to make the most efficient possible use of the limited LVL pump power that is available. In general, the efficiency of such a cavity will increase with the number of round trips during each pump pulse. In the limit of $\mathrm{cw}$ operation, cavity losses are minimized by employing a stable cavity. In the other limit of superradiant operation, the stability of the cavity does not affect the laser output. The "z-cavity" dye laser lies between these limits but the number of round trips per pump pulse is made large enough that cavity stability is an important consideration. For this reason the "z-cavity" is analysed from a quasi-steady state viewpoint. Stability is insured by employing an etalon at Brewster's angle in the telescope leg of the cavity. This etalon compensates for the astigmatism introduced by the off-axis telescope mirrors. 
In the following Section, the basic cavity configuration is analysed as if it were a $\mathrm{CW}$ cavity. One very useful feature of the "z-cavity" design is that the rear leg can be lengthened to accommodate various $l$ ine-tuning and line-narrowing optics without affecting the stability of the cavity.

\subsection{Four-Mirror Equivalent Cavity}

The dye laser cavity, in its basic form without wavelength-tuning elements, consists of four mirrors: a retro-reflecting flat in the rear leg, two folding telescope mirrors, and a flat output mirror. To examine the stability of this four-mirror cavity, it is transformed into

an equivalent two-mirror cavity using the method outlined by Kogelnik ${ }^{30}$. It will be demonstrated that this analysis can be extended to the actual cavity including a prism or a grating.

It is assumed that the folding mirrors of the z-cavity are perfectly reflecting and that they do not aperture the internal cavity mode. The four-mirror cavity can then be schematically represented as an equivalent two-mirror cavity with internal lenses of the same focal lengths as the folding mirrors (Fig. 11).

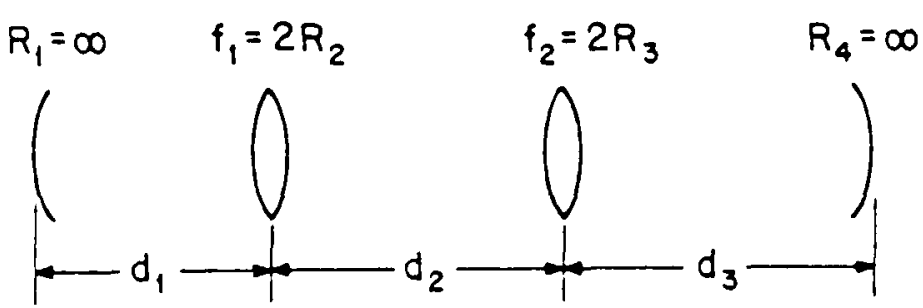

Figure 11. Lens equivalent of the z-cavity. 
In Fig. $11, d_{1}$ and $d_{2}$ are the effective optical path lengths, taking into account the effect of the dye cell in computing $d_{1}$ and the compensation flat in computing $d_{2}$. Note that $d_{1}$ and $d_{2}$, as well as $f_{1}$ and $f_{2}$, will differ in the sagittal (s) and tangential (p) planes due to astigmatism ${ }^{31}$. Specifically, the effective focal lengths of a mirror (or lens) with focal iength $f$ are

$$
\begin{aligned}
& f_{s}=f / \cos \theta \\
& f_{p}=f \cdot \cos \theta
\end{aligned}
$$

where $e$ is the angle of incidence. The effective optical path lengths $d$ of a Brewster-angle flat with refractive index $n$ and thickness $t$ are

$$
\begin{aligned}
& d_{s}=t \sqrt{n^{2}+1} / n^{2} \\
& d_{p}=t \sqrt{n^{2}+1} / n^{4}
\end{aligned}
$$

Each lens-mirror combination can be reduced to an appropriatelyplaced mirror as shown in Fig. 12. 

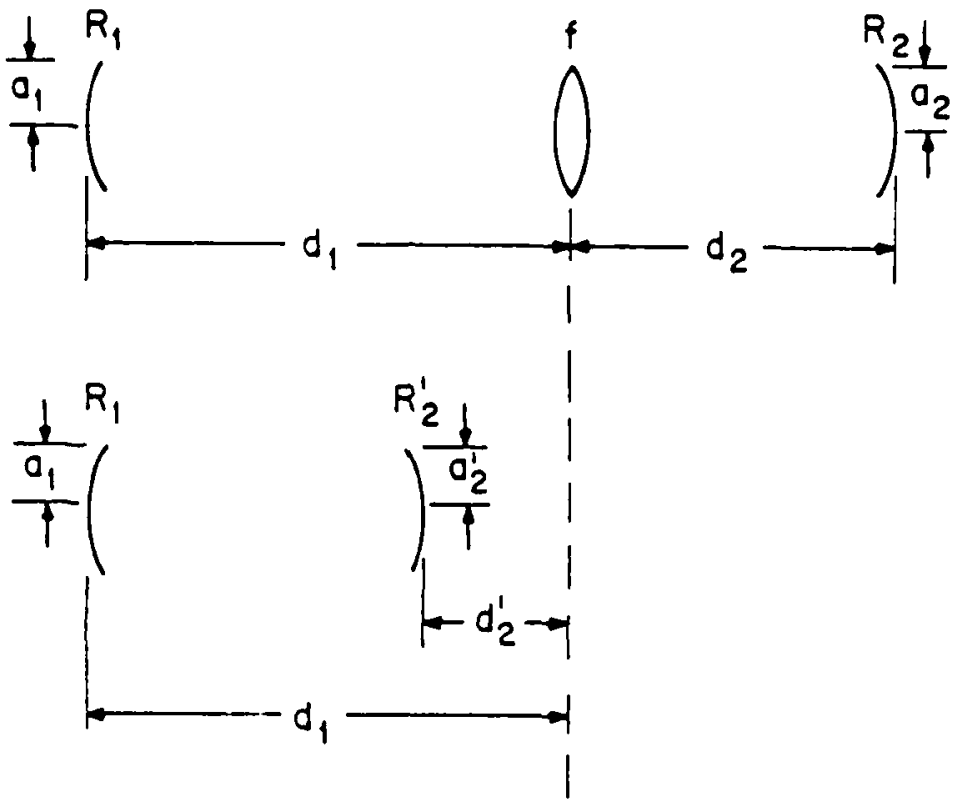

Figure 12. Cavity representation.

From the general formula for the beam transformation by a 1 ens 30 , as given in Fig. 13,

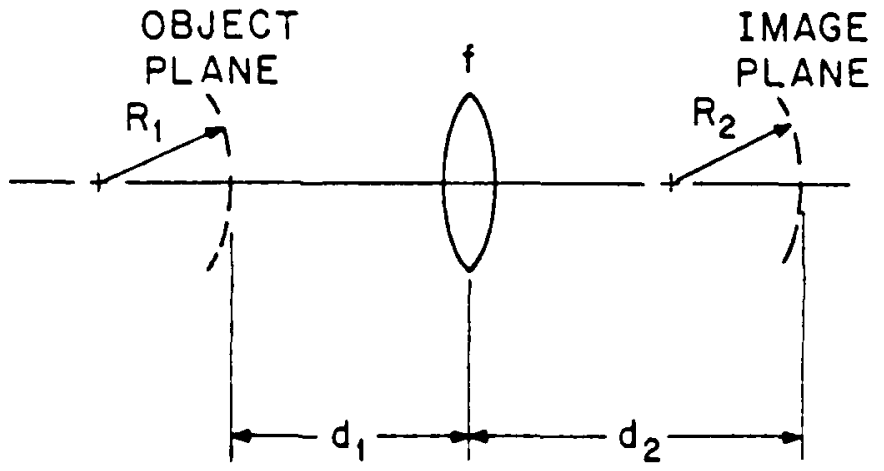

$$
\begin{gathered}
\frac{1}{d_{1}+R_{1}}+\frac{1}{d_{2}-R_{2}}=\frac{1}{f} \\
\frac{1}{d_{1}}+\frac{1}{d_{2}}=\frac{1}{f}
\end{gathered}
$$

Figure 13. Beam transformation by a lens. 
$-50-$

it can be shown that

$$
\begin{aligned}
& d_{2}^{\prime}=\frac{f d_{2}}{d_{2}-f} \\
& a_{2}^{\prime}=\frac{f a_{2}}{f-d_{2}} \\
& \text { of } R_{2}=\infty \text { (a flat mirror), } \\
& \qquad R_{2}^{\prime}=\frac{R_{2} f^{2}}{\left(d_{2}-f\right)\left(d_{2}-f-R_{2}\right)} \\
& f-d_{2}
\end{aligned}
$$

For the case of $R_{2}=\infty$ (a flat mirror),

Returning to the schematic representation of the "z-cavity" (Fig. 11), it is evident that each flat mirror-internal lens pair can be reduced in a straightforward manner to a single mirror with the following result:

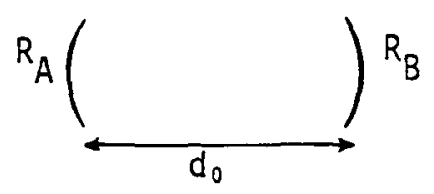

$$
R_{A}=\frac{f_{1}^{2}}{f_{1}-d_{1}}
$$

$$
\begin{aligned}
R_{B} & =\frac{f_{2}^{2}}{f_{2}-d_{3}} \\
d_{0} & =d_{2}-\left(\frac{d_{1} f_{1}}{d_{1}-f_{1}}\right)-\left(\frac{d_{3} f_{2}}{d_{3}-f_{2}}\right)
\end{aligned}
$$


The quantities $d_{1}, f_{1}, d_{2}, f_{2}$ and $d_{3}$ refer to the original two-mirror, two-lens representation of the "z-cavity".

The stability condition for the equivalent two-mirror cavity is:

$$
0 \leq\left(1-\frac{d_{0}}{R_{A}}\right) \quad\left(1-\frac{d_{0}}{R_{B}}\right) \leq 1
$$

Henceforth, we shall designate the quantity between the two inequality signs as the stability factor SF:

$$
0 \leq S F \leq 1
$$

For $\left|R_{B}\right| \geq\left|R_{A}\right|$ there are two ranges of stability:

$A: 0 \leq d_{0} \leq R_{A}: \quad 0 \leq d_{2}-\left(\frac{d_{1} f_{1}}{d_{1}-f_{1}}\right)-\left(\frac{d_{3} f_{2}}{d_{3}-f_{2}}\right) \leq\left(\frac{f_{1}^{2}}{f_{1}-d_{1}}\right)$

$B: R_{B} \leq d_{0} \leq R_{A}+R_{B}:\left(\frac{f_{2}^{2}}{f_{2}-d_{3}}\right) \leq d_{2}-\left(\frac{d_{1} f_{1}}{d_{1}-f_{1}}\right)-\left(\frac{d_{3} f_{2}}{d_{3}-f_{2}}\right) \leq\left(\frac{f_{2}^{2}}{f_{2}-d_{3}}\right)+\left(\frac{f_{1}^{2}}{f_{1}-d_{1}}\right)$

where the choice of signs, $\leq$ or $\geq$, depends on the signs of $R_{B}$ and $R_{A}$. Eqs. 16 and 17 can be solved to determine limits for the unknown design parameter $d_{2}$ :

$$
\begin{aligned}
& \text { A: }\left(\frac{d_{3} f_{2}}{d_{3}-f_{2}}\right)+\left(\frac{d_{1} f_{1}}{d_{1}-f_{1}}\right) \geq d_{2} \leq\left(\frac{d_{3} f_{2}}{d_{3}-f_{2}}\right)+f_{1} \\
& \text { B: } \quad f_{2}+\left(\frac{d_{1} f_{1}}{d_{1}-f_{1}}\right) \leq d_{2} \leq f_{2}+f_{1}
\end{aligned}
$$


In general $\left|\left(\frac{d_{1}}{d_{1}-f_{1}}\right)\right|$ and $\left|\left(\frac{d_{3}}{d_{3}-f_{2}}\right)\right|$ are chosen greater than one. In this case choosing the stability range $B$ (Eq. 19) minimizes $d_{2}$ keeping the dye laser cavity as small as possible. This choice also insures stability independent of $d_{3}$ as long as $\left|\left(\frac{d_{3}}{d_{3}-f_{2}}\right)\right|>1$. It is evident that substituting wavelength tuning and 1 ine narrowing optics for the flat mirror in the back leg of the cavity will not affect stability. Also note that $d_{2}$ is variable over a stable range of width $a \equiv\left(d_{2}\right)_{\max }-\left(d_{2}\right)_{\min }=\left|\frac{f_{1}^{2}}{d_{1}-f_{1}}\right|$; this is the relationship between $f_{1}, d_{1}$ and the minimum tolerance a necessary for construction of the dye laser. For the cavity employed here, this tolerance is $a=1.8 \mathrm{~mm}$.

To insure stability in both planes, astigmatic compensation with a Brewster-angle flat is necessary. By extending the analys is of Kogeinik et $a z^{31}$ to the case of multiple mirrors, one obtains a useful expression relating the flat thickness, $t$, to the folding angle, 20 :

where

$$
2 N t=\sum_{i} R_{i} \sin \theta_{i} \tan \theta_{i}
$$

$$
N=\frac{\left(n^{2}-1\right) \sqrt{n^{2}+1}}{n^{4}} \quad \begin{aligned}
& R=\begin{array}{l}
\text { mirror radius of } \\
\text { curvature }
\end{array} \\
& n=\begin{array}{l}
\text { index of refraction } \\
\text { of flat }
\end{array}
\end{aligned}
$$

Making use of this relation, the cavity is designed to be in the center of the stability ranges of both planes. It is possible to show that the astigmatic effect of the dye cell in the collimated output leg is negligible. 
A slightly different cavity transformation is useful in calculating the dimensions of the output beam. The two telescope mirrors and the rear mirror are transformed, using the same technique as before, to an equivalent mirror at an appropriate distance from the output coupler. The result is the following equivalent cavity.

$$
\begin{aligned}
& \left.R=\infty R^{(}\right) \\
& R^{\prime}=\frac{\left[\frac{f_{1}{ }^{2}-f_{2}^{2}}{\left(f_{2}-d_{3}\right)}\right]}{\left[f_{1}-d_{2}-\left(\frac{f_{2} d_{3}}{f_{2}-d_{3}}\right)\right] \cdot\left[\frac{f_{2}^{2}}{f_{2}-d_{3}}+f_{1}-d_{2}-\left(\frac{f_{2} d_{3}}{f_{2}-d_{3}}\right)\right]} \\
& d_{0}^{\prime}=d_{1}-\frac{f_{1}\left(d_{2}+\frac{f_{2} d_{3}}{f_{2}-d_{3}}\right)}{\left(d_{2}+\frac{f_{2} d_{3}}{f_{2}-d_{3}}\right)-f_{1}}
\end{aligned}
$$

The size of the beam waist, $\nu_{0}$, which in a flat-curved cavity occurs at the flat mirror, is

$$
\omega_{0}=\left(\frac{\lambda d_{0}^{\prime}}{\pi}\right)^{\frac{1}{2}} \cdot\left[\frac{\left(R^{\prime}-d_{0}^{1}\right)}{d_{0}^{\prime}}\right]^{\frac{1}{4}}
$$

The flat mirror in the equivalent cavity corresponds directly to the output coupler; therefore a waist occurs at the "z-cavity" output mirror of size is found from Eq. 22. 
If the effect of the finite thickness of the output mirror is neglected, then the size of the output Gaussian beam of the dye laser at a distance $z$ from the output mirror is

$$
w(z)=w_{0}\left[1+\left(\frac{\lambda z}{\pi \omega_{0}^{2}}\right)^{2}\right]^{\frac{1}{2}}
$$

The diffraction-limited angular divergence of the output beam in the far field $z>\frac{w_{0}}{\lambda}$ is described by the angle se where

$$
1 \pm=\frac{\lambda}{i_{0}}
$$

\subsection{Design Specifications}

The detemination of design specifications proceeds from the choice of telesocpe mirrors. This choice is affected by the trade-off between large internal beam expansion and short round trip path length. The round trip path length can be well be? ow $1 \mathrm{~m}$ for a $10 \times$ internal beam expansion where mirrors of focal lengths $f_{1}=12.5 \mathrm{~mm}$ and $f_{2}=125 \mathrm{~mm}$ are employed. Using a $12.7 \mathrm{~mm}$ thick fused silica compensation flat $\left(n=1.45\right.$ at $\lambda=.9 \mathrm{im}^{32}$ ), a folding angle of $23=23^{\circ}$ is required (Eq. 20).

To allow adequate space for 311 of the cavity optics, the distance $d_{1}$ between the output coupler and the first folding mirror $f_{1}=12.5 \mathrm{~mm}$ is chosen to be $d_{l}=100 \mathrm{~mm}$. The dye cell, which consists of a $.5 \mathrm{~mm}$ thick dye volume enclosed by two $2.25 \mathrm{~mm}$ thick fused silica windows of approximately the same refractive index as the dye solution, is modelled 
as a $5 \mathrm{~mm}$ thick Brewster-angle fused silica flat. Then, using Eq. 11 to find the effective optical path lengthis of the dye cell and compensation flat, the distance $d_{2}$ between the two telescope mirrors is calculated so that the effective optical path lengths in the $s$ and $p$ planes are in the center of the stability range given in Eq. 19. The result is $c_{2}=$ $145.0 \mathrm{~mm}$. The cavity will be stable as long as the effective optical path lengths of the rear leg of the cavity $d_{3}$ eff satisfy

$$
\begin{aligned}
& d_{3_{\text {eff }}} \geq \frac{f^{2} s}{2}=63.8 \mathrm{~mm} \\
& d_{3_{\text {eff }}} \geq \frac{f^{{ }_{2}}}{2}=61.2 \mathrm{~mm}
\end{aligned}
$$

Using this design method it is found that the stability factor SF (Eq. 15b)

$$
S F \equiv\left(1-\frac{d_{0}}{R_{A}}\right)\left(1-\frac{d_{0}}{R_{B}}\right)=\left(1-\frac{d_{0}}{R^{1}}\right)
$$

is equal to .50 in both the $s$ and $p$ planes. According to Eqs. 15 , the cavity is therefore stable.

For the cavity constructed, $d_{\text {eff }} \cong d_{\text {eff }} \cong 75 \mathrm{~mm}$ and the beam waist of the cavity mode at the output mirror $\omega_{0}$ is found from Eqs. (21) and (22) to be

$$
\omega_{0}=.16 \mathrm{~mm}
$$

at an output wavelength of $\lambda=.9 \mathrm{~mm}$. For this wavelength the angular divergence $i E$ is found from $E q$. (24) to be 
Design values for a cavity with a $20 x$ internal beam expansion were also calculated. They assume the same $12.7 \mathrm{~mm}$ thick compensation wedge and are shown in Table 1 along with the design values for the 10x cavity. Wavelength-dependent quantities assume $\lambda=.9: \mathrm{um}$

The round-trip time $2 L / C$ of the $10 x$ cavity is seen to be less than 2.5 ns and that of the $20 x$ cavity with $d_{3}$ eff $\cong 150 \mathrm{~mm}$ is less than $4 \mathrm{~ns}$. In either case, the quasi-steady-state approximation that many round trips occur during each pump pulse is valid.

In addition to the quasi-steady-state assumption, two approximations were made in the cavity analysis. These were that no losses occur at the telescope mirrors and that they do not aperture the beam. The justification of these approximations is dependent on the optics used. The telescope mirrors are coated for maximum reflectivity at normal incidence from $.72-1.15 \mathrm{jm}$. At the angle of incidence $\mathrm{z}=11.5^{\circ}$, transmission losses are negligible throughout the wavelength range of operation of the dye laser. The diameter of the first folding mirror is $12.7 \mathrm{~mm}$. The longer focal length telescope mirror and the compensation flat are $25.4 \mathrm{~mm}$ in diameter. Given the calculated mode size (Table 1) it is clear that the optics do not aperture the internal cavity mode.

All of the elements of the dye laser as well as the pump folding mirror and lens (Fig. 9) were bolted to a $\frac{1}{2} "$ thick aluminum plate. The plate was drilled using a numerical drill press with a precision of $=.0005^{\prime \prime}$. Taking into account the inaccuracies encountered in conversion 


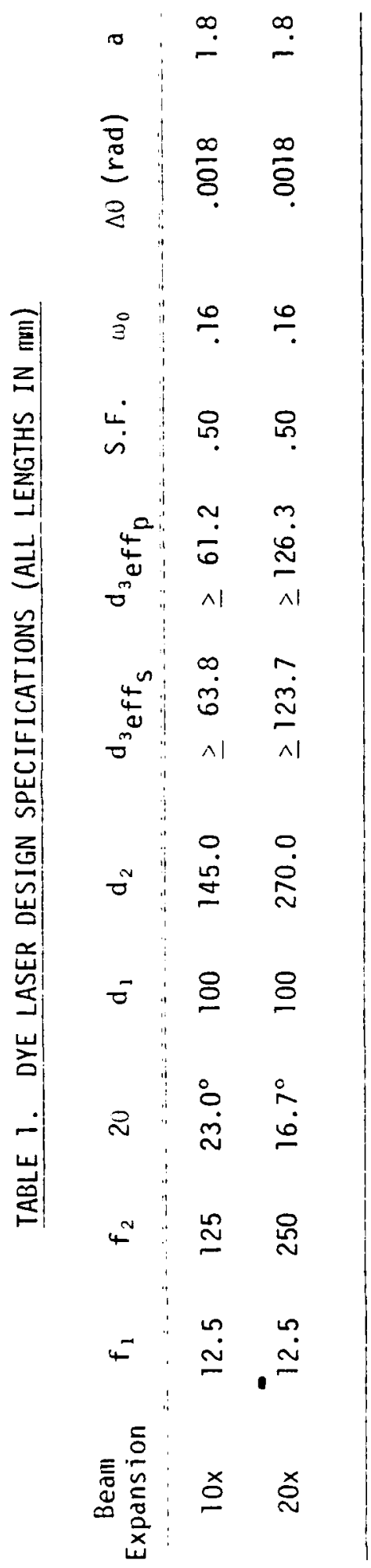

$-$ 
from optical surfaces to mirror mount hole positions, the placement of the most criical dye laser optics should be within the tolerance $\pm \mathrm{a} / 2= \pm .90 \mathrm{~mm}$ found from Eq. (9). Ardel Kinematic U100MP Mounts (resolution 15 urad) were used for the output mirror and the first folding mirror. The other folding mirror was mounted in an Oriel 1435 Mount (resolution 5 urad). Higher resolution Aerotech AOM110-2 Mounts (resolution .7 urad) were found to be necessary for the pump mirror and the rear tuning mirror.

\subsection{Wavelength Tuning}

The dye laser that was built and tested first employed a $60^{\circ}$ glass prism and a flat rear mirror for wavelength tuning. In this Section the angular dispersion of the prism is calculated as well as that of a commercially-available diffraction grating. Although it is prohibitively difficult to calculate the output linewidth given the dispersion, it is clear that as the dispersion increases the output linewidth will decrease. This relationship stems from the homogeneous nature of the dye medium.

The following relation can be found for an isoceles dispersing prism with apex angle $x^{33}$ (Fig. 14)

$$
j=\theta_{i}+\theta_{t}-\alpha
$$

where $f$ is the angular deviation of an incident ray. In terms of $y_{j}$ and $\lambda$ this expression is

$$
f=\theta_{i}+\sin ^{-1}\left[\sin i\left(n^{2}-\sin \theta_{i}\right)^{\frac{1}{2}}-\sin \theta_{i} \cos a\right]-a
$$




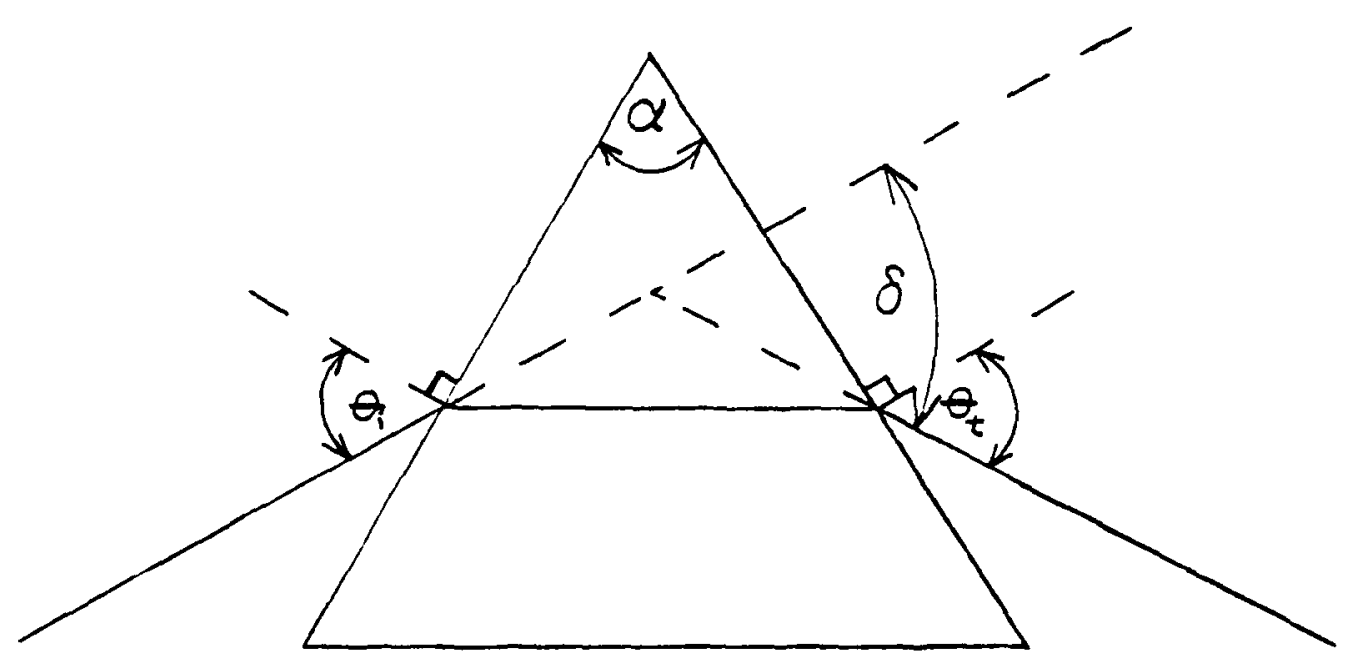

Figure 14. Isoceles orism at minimum deviation. 
In Fig. 14, the incident ray traverses the prism at minimum deviation in which case $\vec{\theta}_{i}=\hat{\theta}_{t}$ and the ray travels within the prism parallel to its base. The angle of incidence, $\theta_{j}$, for this case is

$$
\theta_{i}=\sin ^{-1}\left[n \sin \frac{\alpha}{2}\right]
$$

and the angular deviation, $\Xi_{m}$, of the incident ray is

$$
\delta_{m}=2 \theta_{i}-x
$$

The prism is employed in the dye laser cavity close to minimum deviation to minimize its round-trip reflection loss. The angle of incidence $\partial_{j}$ is fixed while the angle of transmission $\hat{\gamma}_{t}$ varies slightly as the rear cavity mirror is rotated for wavelength tuning. For this type of operation, the angular diviation can be written

$$
\hat{c}=2 \theta_{j}+\varepsilon-x
$$

where $\varepsilon \ll \theta_{i}$ for the entire tuning range of the dye laser.

Differentiating both sides of Eq. 27 with respect to wavelength, $\lambda$, and rearranging terms results in the following expression for the wavelength dependence of the angular deviation:

$$
\frac{d \delta(\lambda)}{d \lambda}=\frac{n(\lambda) \sin \alpha}{\left(n^{2}(\lambda)-\sin ^{2} \theta_{i}\right)^{\frac{1}{2}} \cos \left(\partial(\lambda)-\theta_{i}+\alpha\right)} \cdot \frac{d n(\lambda)}{d \lambda}
$$


Using Eq. 30, this can be written as

$$
\frac{\mathrm{d} \delta(\lambda)}{d \lambda}=\frac{n(\lambda) \sin \alpha}{\left(n^{2}(\lambda)-\sin ^{2} \partial_{i}\right)^{\frac{1}{2}} \cos \left(\theta_{i}+\varepsilon(\lambda)\right)} \cdot \frac{\mathrm{dn}(\lambda)}{d \lambda}
$$

The above expression is the angular dispersion of the prism. do/d $\lambda$ al so corresponds exactly to the variation of the angle of retroreflection of the rear cavity mirror with wavelength. Typically $\cos \left(g_{i}+\varepsilon\right) \cong \cos \left(\theta_{i}\right)$ so that Eq. 32 can be easily solved for a given prism once $\mathrm{dn} / \mathrm{d} \lambda$ is known. In the case of the $x=60^{\circ}$ glass prism employed in the dye laser, the dispersion is derived from the expression for the refractive index $n$ of fused silica given by Moses 32 ( $\lambda$ in $\mu \mathrm{m} ; \mathrm{T}=298^{\circ} \mathrm{K}$ )

$$
\begin{aligned}
n(\lambda) & =\left[A+\frac{B}{\lambda^{2}-C}+\frac{C}{\lambda^{2}-E}\right]^{\frac{1}{2}} \\
A & =2.978645 \\
B & =.008777808 \\
C & =.010609 \\
D & =84.06224 \\
E & =96.00000
\end{aligned}
$$

Taking the derivative of Eq. 33 with respect to wavelength vields the following formula:

$$
\frac{d n(\lambda)}{d \lambda}=-\lambda \cdot\left[A+\frac{B}{\lambda^{2}-C}+\frac{D}{\lambda^{2}-E}\right]^{-\frac{1}{2}} \cdot\left[\frac{B}{\left(\lambda^{2}-C\right)^{2}}+\frac{D}{\left(\lambda^{2}-E\right)^{2}}\right]
$$


Using Eqs. 32 and 34, the angular dispersion of the $60^{\circ}$ prism at $\lambda=.9$ um is found to be

$$
\frac{d \delta}{d \lambda}=-2.1 \times 10^{-2} \mathrm{rad} / \mu \mathrm{m}
$$

where the angle of incidence is calculated from Eqs. 28 and 33 to be that of minimum deviation:

$$
\theta_{i}=46.5^{\circ}
$$

Brewster's angle at $\lambda=.9$ im is $\Theta_{B}=55.4^{\circ}$. As a result, a TM wave incident on the prism will suffer a .5\% reflection loss per surface, i.e. the round-trip loss will be $2 \%$ greater than if a Brewster-angle prism had been used.

A considerabiy higher angular dispersion can be achieved with a Littrow-mounted diffraction grating. When the grating is used in first order

$$
\sin \theta_{i}=\sin \hat{\theta}_{r}=\frac{\lambda}{2 d}
$$

where $\theta_{j}$ and $\theta_{r}$ are the angles of incidence and reflection and $d$ is the line spacing. The angular dispersion is

$$
\frac{d \theta_{r}}{d \lambda}=\left(2 d \cos \theta_{r}\right)^{-1}
$$

For a 1200 groove per millimeter grating operating at $\lambda=.9 \mathrm{um}$, the angular dispersion is calculated to be 


$$
\frac{d \theta_{r}}{d \lambda}=7.1 \times 10^{-1} \mathrm{rad} / \mathrm{m}
$$

and the angle of incidence is

$$
\theta_{i}=32.7^{\circ}
$$

A PTR U-TF-32 gold-coated diffraction grating should provide this angular dispersion, which is thirty-four times that of the $60^{\circ} \mathrm{glass}$ prism, with a peak efficiency of $90 \%$ at $\lambda=1 \mu \mathrm{m}$.

\subsection{Dye Cell and Flow System}

During laser operation, the dye solution must be circulated to prevent losses due to thermal degradation of the dye, localized dye heating, and the adherence of dye particles to the cell windows. The most critical part of the dye flow system is the dye cell. A unique cell design has been implemented which confines the dye to a very narrow stream in the active region.

The requirement of a narrow flow stream in the active region is due primarily to the optical pumping configuration. The most efficient pumping method is to bring the polarized pump beam into the dye laser cavity along its axis by using a dichroic mirror. The pump beam is preferably transformed by a lens outside the cavity so that it exactly matches the Gaussian cavity mode. In this way the entire excited dye volume contributes to the internal cavity field. In addition, there is no pump beam reflection loss at the Brewster-angle dye cell. However, 
this axial pumping method is impractical because of the difficulty in obtaining a suitable dichroic mirror.

The alternative arrangement which has been chosen is shown in Fig. 9. The LVL pump beam is folded toward the dye cell by a totallyreflecting flat mirror. A lens is employed to focus the pump beam onto the dye cell. The angle between the pump beam and the cavity axis is made as small as possible consistent with the geometry of the mirror mounts. This reduces the reflection losses at the dye cell and maximizes the spatial overlap between the focussed pump beam and the cavity mode within the dye cell. In addition to maximizing the efficiency of the energy transfer from the pump to the internal cavity field, this minimizes the absorption loss presented by the unexcited dye volume that intersects the cavity mode. By making the dye stream as narrow as possible, this unexcited dye volume and the associated loss is minimized. Practical considerations determine the minimum thickness.

The most common method of producing a very narrow dye stream is to use an unconfined "jet stream nozzle" of some kind. ${ }^{34}$. It is possible, by using solvents of suitable viscosity and surface tension, to produce a non-turbulent jet with exactly parallel surfacos. The solution is normally collected by allowing the unconf ec $\therefore$ eam to enter a large tube and drain off to a reservoir. Unconfined flow was not used because it restricts the choice of dye solvents and it can be difficult to operate in a reliable manner. 


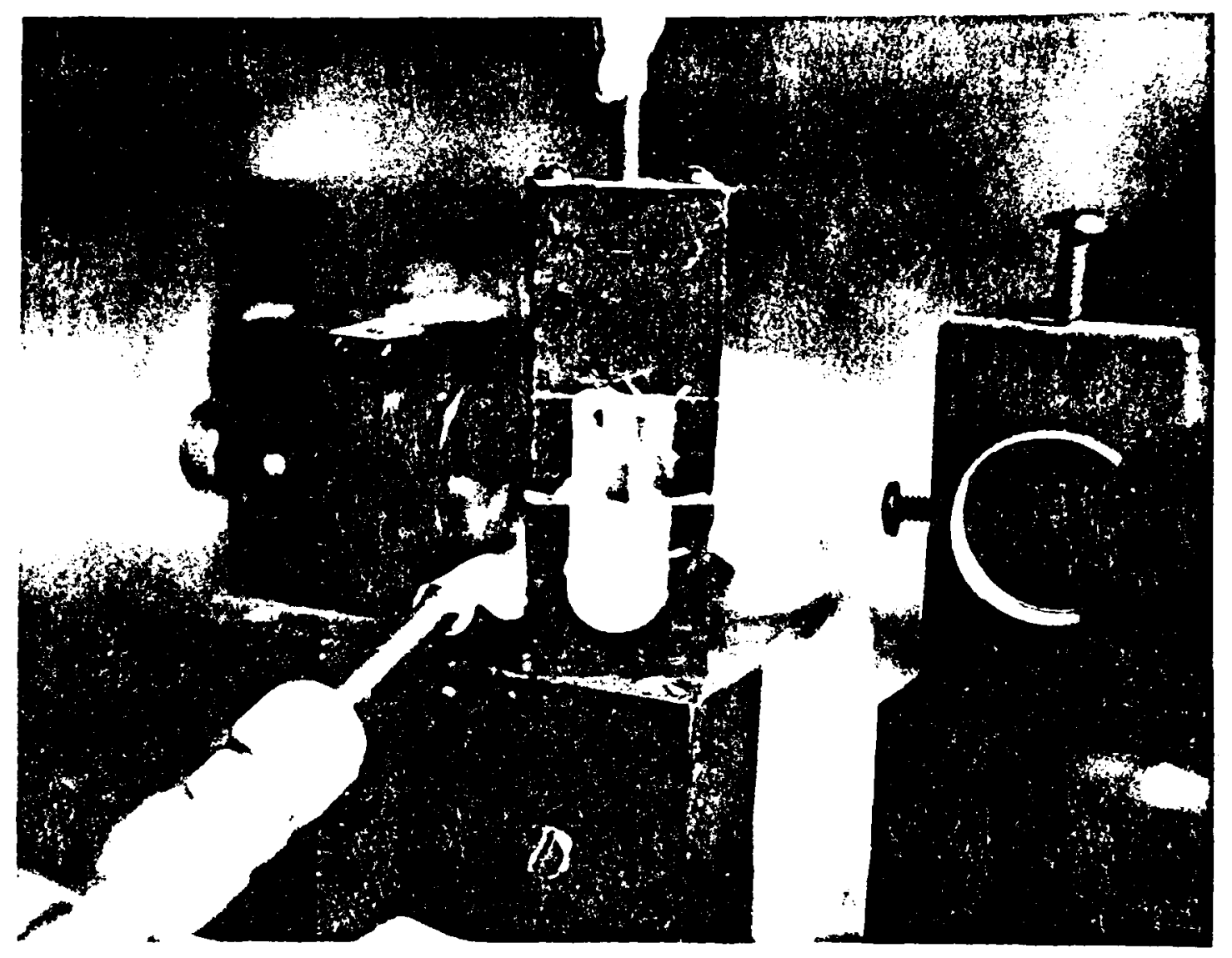

Figure 15. The dye cell. 


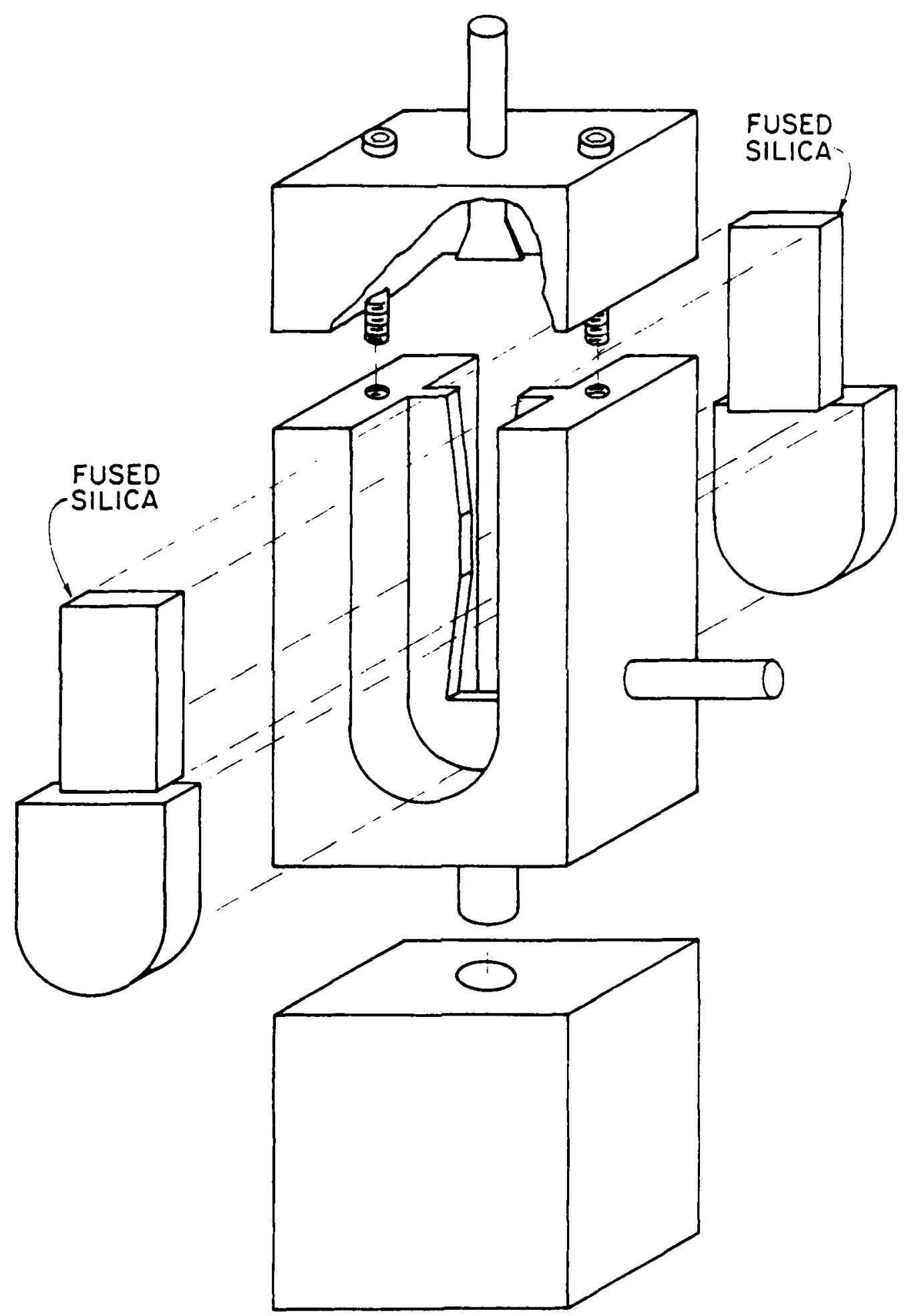

Figure 16. Exploded view of dye cel1. 
The dye cell that was designed and built is pictured in Fig. 15 and is drawn in an exploded view in Fig. 16. The dye solution flows into the cell through a jet nozzle that consists of a flattened $3 / 8^{\prime \prime}$ stainless steel tube that is approximately $5 \mathrm{~mm} \times .5 \mathrm{~mm}$ at the flared end (Fig. 17). This end sits atop a channel that is cut in an aluminum cell. The channel (Fig. 18) is enclosed by $2.25 \mathrm{~mm}$ thick etalons and is $3 \mathrm{~mm}$ wide by $.5 \mathrm{~mm}$ thick in the active region. The channel was milled with dental burrs. The dye solution flows out of the side of the main cell body well below the active region. The dye solution is circulated between the cell and a reservoir by a Micropump Model 040-331-200 vol tage-controlled pump.

It is possible to estimate the flow rate necessary to prevent significant heating of the dye solution. It is initially assumed that the active dye volume is essentially fixed in space during each pump putse ( $\approx 30 \mathrm{~ns})$. Then for a quantum efficiency (ratio of the number of radiated photons to absorbed photons) of the dye solution, Q, the change in temperature of the active dye volume per pump pulse iT is

$$
\dot{-T}=\left(\frac{E}{V \cdot C_{p}}\right)\left(\frac{e^{-\lambda} p}{p}\right) Q\left[1+\left(\frac{\lambda_{p}}{\lambda^{-\lambda} p}\right)\left(\frac{1-Q}{Q}\right)\right]
$$

where $E$ is the energy absorbed from the Dumo pulse, $V$ is the active dye volume, $C_{p}$ is the heat capacity of the dye solution, ${ }_{p}$ is the pump wavelength, and ' $e$ is the wavelength of the dye solution emission. 
$-68-$

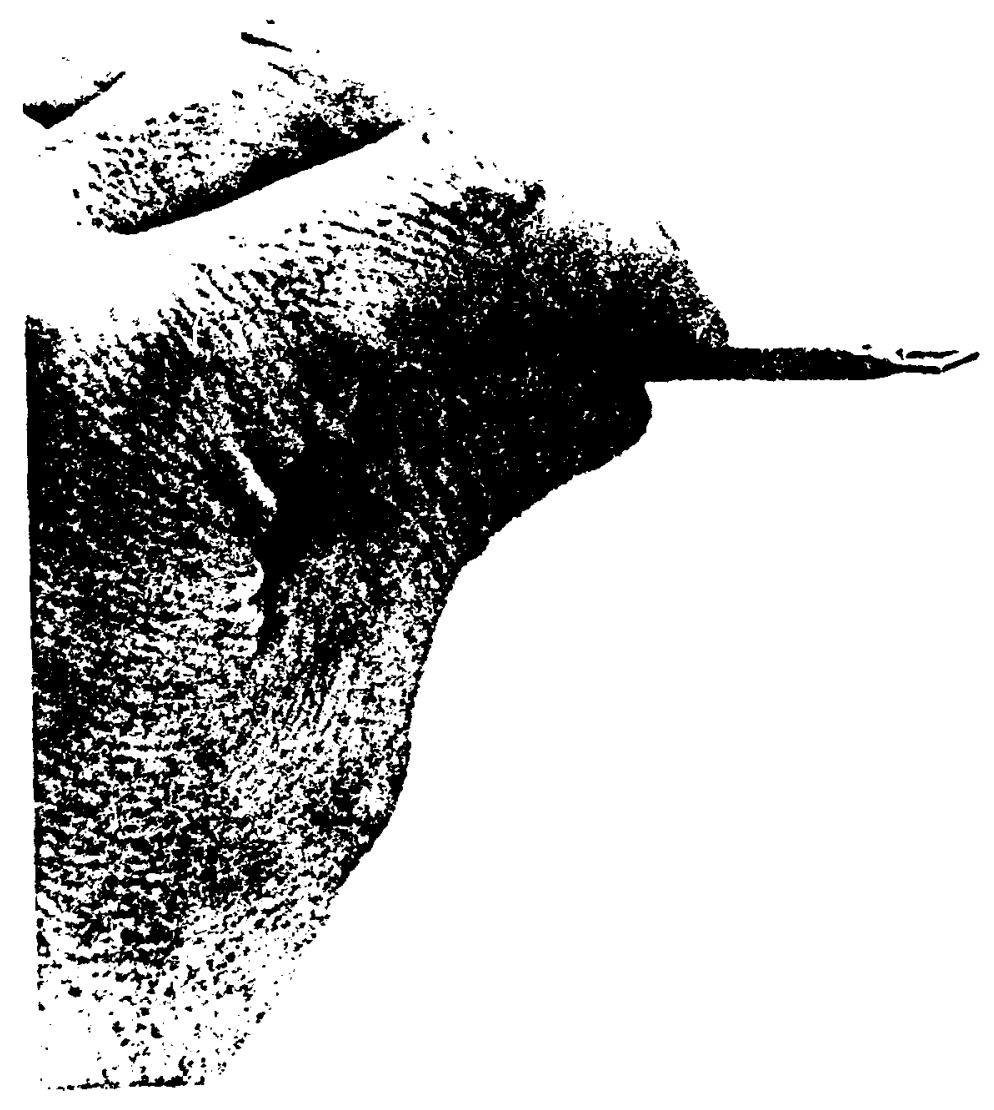

Figure 17. Dye flow nozzle. 


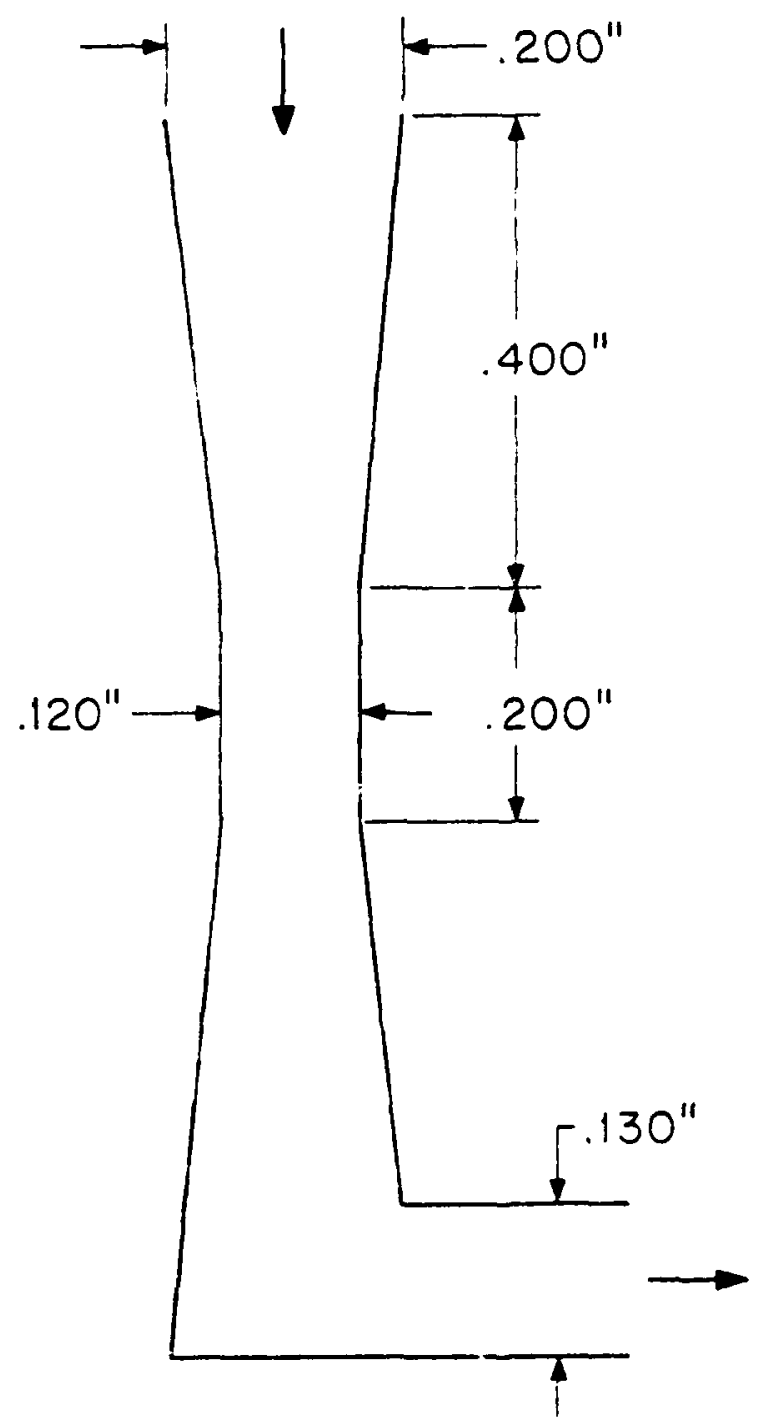

Fig'are 18. Dye cell channel.

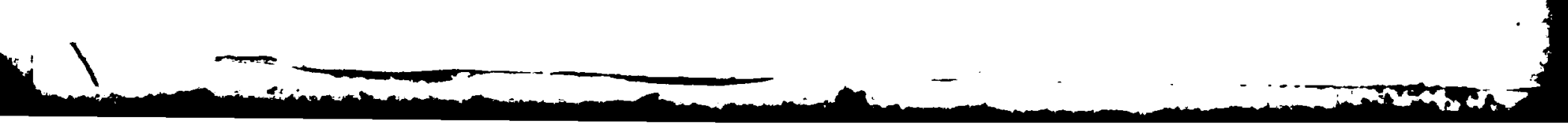


A representative calculation for $\Delta T$ is now presented. The excited dye volume is taken to be the product of the dye cell thickness and the cross-sectional area of the cavity mode at the cell $\left(\approx-\omega_{0}{ }^{2}\right)$. The heat capacity of the dye solution is about one-half that of water; this value is representative of the specific heats of typical organic solvents. For a quantum efficiency of $90 \%$ and an absorbed energy of $100 \% \mathrm{~J}$, the temperature change per pulse of the active dye volume with the dye laser operating at a wavelength of .9 im will be approximately

$$
\Delta T=.4^{\circ} \mathrm{C}
$$

This relatively low figure indicates that thermal effects within the dye cell, including thermal lensing, should be negligible. For a detailed calculation of thermal lensing the reader is referred to Morey ${ }^{35}$.

To insure that the above single-pulse temperature rise is the maximum temperature increase in the active dye region, the dye solution nust move at least one diameter $(.32 \mathrm{~mm})$ in the time between pump pulses. At a $2 \mathrm{KHz}$ PRF, this requires a linear flow rate of $64 \mathrm{~cm} / \mathrm{sec}$ and a volumetric flow rate of $.96 \mathrm{~cm}^{3} / \mathrm{sec}$ in the channel that is $.5 \mathrm{~mm} \times 3 \mathrm{~mm}$ in the active region. Typically, operational flow rates are many times greater than these minimum values. 


\subsection{Experimental Results}

Two infrared dyes have been utilized in the dye laser: HITC-P 36 and $I R-140^{37}$. The latter was tested in two solvent mixtures. Table 2 is a list of the peak abosrption wavelengths and the absorption coefficients at $.7229, \mathrm{~m}$ for the solutions used. These values were taken from absorption spectra measured using a Cary I4R Spectrophotometer.

The dye laser was initially operated broadband as a four-mirror $z$-cavity with no wavelength-tuning element. The output mirror transmission was $T=.10$. With the HITC-P dye solution, peak output power was observed at $8041 \AA$. This corresponds to a difference-frequency with a wavelength $(\approx 7.2 \mathrm{im})$ substantially further into the infrared than the long-wavelength transmission limit of $\mathrm{LiNbO}_{3}$. IR-140 in a solution of $50 \%$ dimethylsulfoxide (DMSO) and 50\% ethylene glycol (EG) produced peak output at $8695 \AA$, corresponding to difference-frequency wavelength of $\because=4.3 \mathrm{~cm}$. In both cases the dye concentration was selected for $90^{\circ}:$ absorption of the pump beam incident on the dye volume.

\section{TABLE 2. ABSORPTION DATA FOR OYE SOLUTIONS}

\begin{tabular}{|c|c|c|c|}
\hline Dye & $\begin{array}{l}\text { Solvent } \\
\text { Mixture }\end{array}$ & $\begin{array}{l}\text { Xpeak (im) } \\
\text { absorption }\end{array}$ & $\therefore \quad \begin{array}{r}a=.7229 \\
\left(M^{-1} \mathrm{~cm}^{-i}\right)\end{array}$ \\
\hline HITC-P & $\begin{array}{l}16 \% \text { DMSO } \\
84 \% \text { EG }\end{array}$ & .75 & $1.3 \times 10^{5}$ \\
\hline IR -140 & $\begin{array}{l}50 \% \text { DMSO } \\
50 \% \text { EG }\end{array}$ & $.74-.75$ & $7.3<10^{4}$ \\
\hline IR -140 & $100 \%$ DMSO & $.82-.83$ & $4.7 \times 10^{4}$ \\
\hline
\end{tabular}


The broadband half power bandwidth was about $30 \AA$ for both dye solutions. This was reduced to less than $10 \AA$ by including a $60^{\circ} \mathrm{glass}$ prism in the rear leg of the cavity as indicated in Fig. 9 . In this configuration the output power obtained with IR-140 in a $50 \%$ DMSO $50 \%$ EG solution was constant as the output was tuned from $8629-3715 \AA$.

The tuning range of the dye laser with IR-140 was significantly red-shifted by changing the solvent mixture to $100 \%$ DMSO. Note that such a shift might be suggested by the absorption peaks indicated in Table 2. The laser was operated at four concentrations of IR-140 in DMSO corresponding to pump absorption of $95 \%, 90 \%, 85 \%$, and $80 \%$. The output energy vs. input pump energy is plotted in Fig. 19. The energy slope Efficiency was about $2.2 \%$ and the highest energy conversion efficiency, $1.2 \%$, occurred at the $90 \%$ absorption concentration. The tuning range and the output bandwicth did not vary appreciably with the dye concentration. The tuning range over which the dye laser out put was at least half of the peak output energy was approximately 3630-8910 $\AA$. The half power bandwidth was $3 \AA$. The threshold pump energy was between $30-40 ; \mathrm{J} /$ pulse.

The efficiency of the laser was somewhat lower, than anticidated. Outout mirrors with transmissions of $T=.03, T=.50$ and $T=.90$ resulted in still lower efficiency. This problem may have had several sources including the following: the low optical quality of the prism used in the experiment; the pump losses at the 1" diameter pump folding mirror and focussing lens; and impurities in the dye solution. In addition, the efficiency was found to be a strong function of cavity 


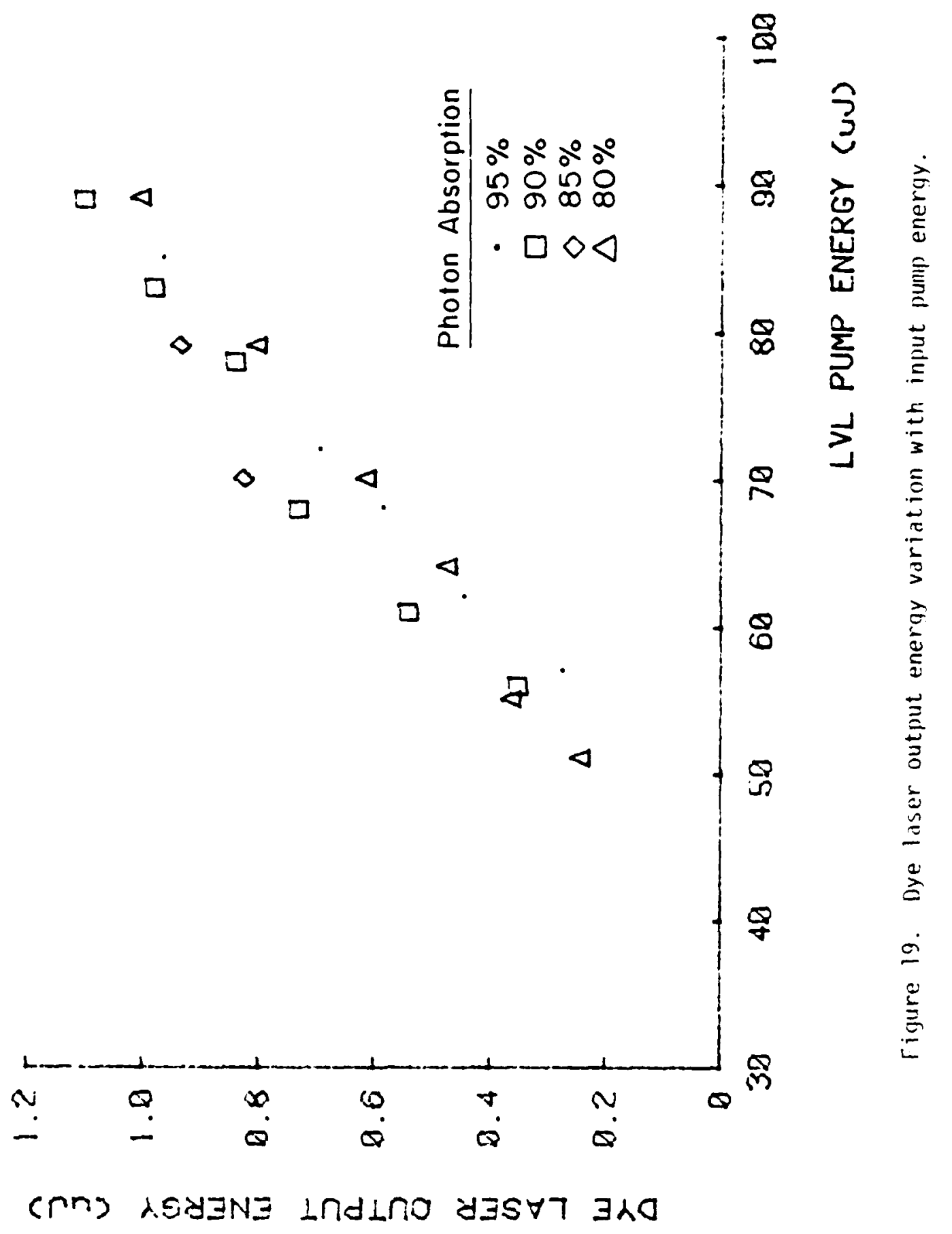


alignment. For the experiment of Fig. 19, the cavity was aligned using a $2.5 \mathrm{~mW}$ helium-neon visible laser. Once laser emission was detected, the alignment was further adjusted for peak output. However, subsequent experiments demonstrated that this method did not always converge on the most efficient alignment. Optimum alignment was obtained by placing an optical flat in the rear leg of the cavity during dye laser operation. The optical flat is olaced at a small anale to the beam axis, and an infrared image plate is used to display the spatial symmetry of the internal field of the dye laser. The alignment is tuned so that the internal field is circularly symetric; then removing the flat and adjusting the alignment for peak output will result in optimal efficiency.

The half power pulsewidth of the dye laser increased with the pump power. Pulses of 25 ns width were observed for 30 ns pump pulses of about $100 \mathrm{u}$. Dye laser pulse widths under $10 \mathrm{~ns}$ were observed near threshold. Fig. 20 is a typical dye laser pulse for a oump pulse enersy of about $75 \mathrm{iJ}$.

The dye laser was also operated with a gold coated diffraction grating (PTR U-TF-32) as shown in Fig. 10. The output bandwidth was. as expected, far lower than that achieved with the prism. The bandwidth was less than $7 \AA$. The medsurement was limited by the resolution of the monochromator. 


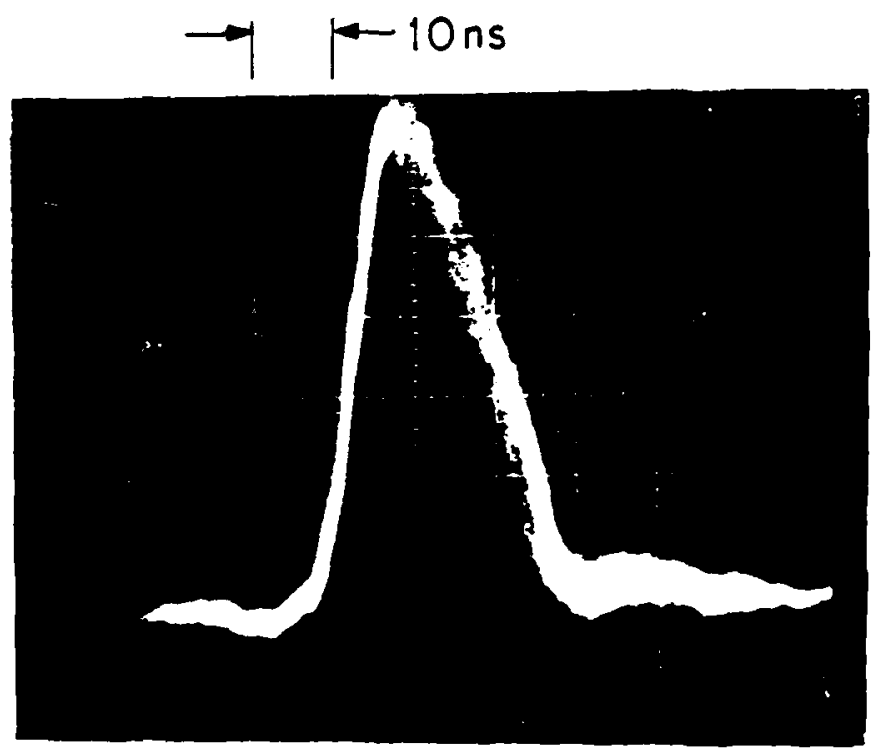

Figure 20. Dye laser output waveform. 


\section{THE MIXING SYSTEM}

In this Chapter the mixing system is analyzed. First the important properties of the crystal are discussed. In Section 5.2 the temperature control required for phase-matching is detailed. The complete optical

system is presented in Section 5.3 and in the final Section the potential system output is calculated.

\subsection{Properties of Lithium Niobate}

The mixing sources, the LVL and the dye laser, are two basic components of the infrared difference-freuuency system. The third is the nonlinear crystal in which the mixing occurs. Lithium niobate has all of the properties of a suitable mixing crystal and is a distinctly attractive choice from the points of view of phase-matchability and damaje threshold.

Crystais of lithium niobate, which is a uniaxial crvstal, are available in good optical quality and useful size and have a large nonlinearity. It has a useful transmission range $(T>80 \%)$ from about $0.4-4.5 \mathrm{~m}$. Sy controlling the temperature of the crystal, $90^{\circ}$ phasenatching can be achieved. Here Type I phase-matching must be employed; the dye laser and generated infrared beams must propagate in the srystal as ordinary waves and the LVL beam must propagate as an extraordinary wave. Phase-matching at $90^{\circ}$ from the optic axis has two distinct advantages. First, the beam interaction length in the crystal is not limited by walk-off because there is no double refraction. 
Second, the allowable divergence of the mixing beams is maximized. This method of phase-matching also has a positive effect on the optical damage threshold of the lithium niobate crystal. Laser damage, in the form of a track through the crystal of different refractive index that is induced at high power levels, is seen to rapidly anneal out at temperatures above $180^{\circ} \mathrm{C}$. In the following Section, it is demonstrated that the phase-matching temperatures for the sets of frequencies involved here are well above the annealing temperature. The surface damage threshold of lithium niobate is relatively high. It has been measured to be $1.2 \times 10^{3} \mathrm{~N} / \mathrm{cm}^{2}$ for a $30 \mathrm{~ns}$ pulse at $1.06 \mathrm{um}^{38}$.

\subsection{Temperature Control of the Mixing Crystal}

In order to determine the crystal temperature reauired to phasematch a particular set of mixing frequencies, the ordinary and extraordinary refractive indices must be known as functions of temoerature at those frequencies. Numerical calculation can then be used to solve for the temperature that satisfies the collinear phase-match conditon:

$$
\Delta k=\frac{1}{c}\left[n_{e}\left(\lambda_{3}, T\right) \omega_{3}-n_{0}\left(\lambda_{2}, T\right) \omega_{2}-n_{0}\left(\lambda_{1}, T\right) i_{1}\right]=0
$$

Hobden and Warner 39 have determined two temperature-denendent Sellmeier equations for the refractive indices of stochiometric lithium niobate between $0.4 \mu \mathrm{m}$ and $4.0 \mathrm{\mu m}$, valid up to $600^{\circ} \mathrm{C}$. These are $(\lambda$ is the wavelength in nanometers and $T$ is the temperature in degrees Kelvin): 


$$
\begin{aligned}
& n_{0}^{2}=4.9130+\frac{1.173 \times 10^{5}+1.65 \times 10^{-2} T^{2}}{\lambda^{2}-\left(2.12 \times 10^{2}+2.7 \times 10^{-5} T^{2}\right)^{2}}-2.78 \times 10^{-8} \lambda^{2} \\
& n_{e}^{2}=4.5567+2.60510^{-7} T^{2}+\frac{0.97010^{5}+2.7010^{-2} T^{2}}{\lambda^{2}-\left(2.01 \times 10^{2}+5.4 \times 10^{-5} \mathrm{~T}^{2}\right)^{2}}-2.24 \times 10^{-8} \lambda^{2}
\end{aligned}
$$

Fig. 21 is a plot of the calculated phase-matching temperature as a function of the dye laser wavelength and the corresponding differencefrequency wavelength for most of the desired mixing range. The data points are determined using Eqns. 38 and 39 . Fig. 22 is an expanded region of Fig. 21 for dye laser wavelengths from 890-900 nm.

Eqns. 39 apply to stochiometric material and in general cannot be applied with sufficient accuracy to commonly available lithium niobate crystals. This is due to the small variation in composition between crystals. In general, the phase-match temperatures calculated from Eqn. 38 and 39 will differ from the actual values by a constant offset temperature. The magnitude of this offset is not expected to exceed $50^{\circ} \mathrm{C}^{40}$. Typically, if the offset temperature has been determined experimentally at one or more points, the resultant temperature-shifted phase-matching curves are in excellent agreement with experimental data. This determination is possible through the method of spontaneous parametric scattering as detailed by Byer and Harris ${ }^{40}$ (see Appendix A).

Having determined the correct phase-match temperature, it is important to find the degree of temperature control that is necessary to maintain efficient generation for a fixed set of mixing frequencies. The temperature bandwidth is defined as the temperature range over which 


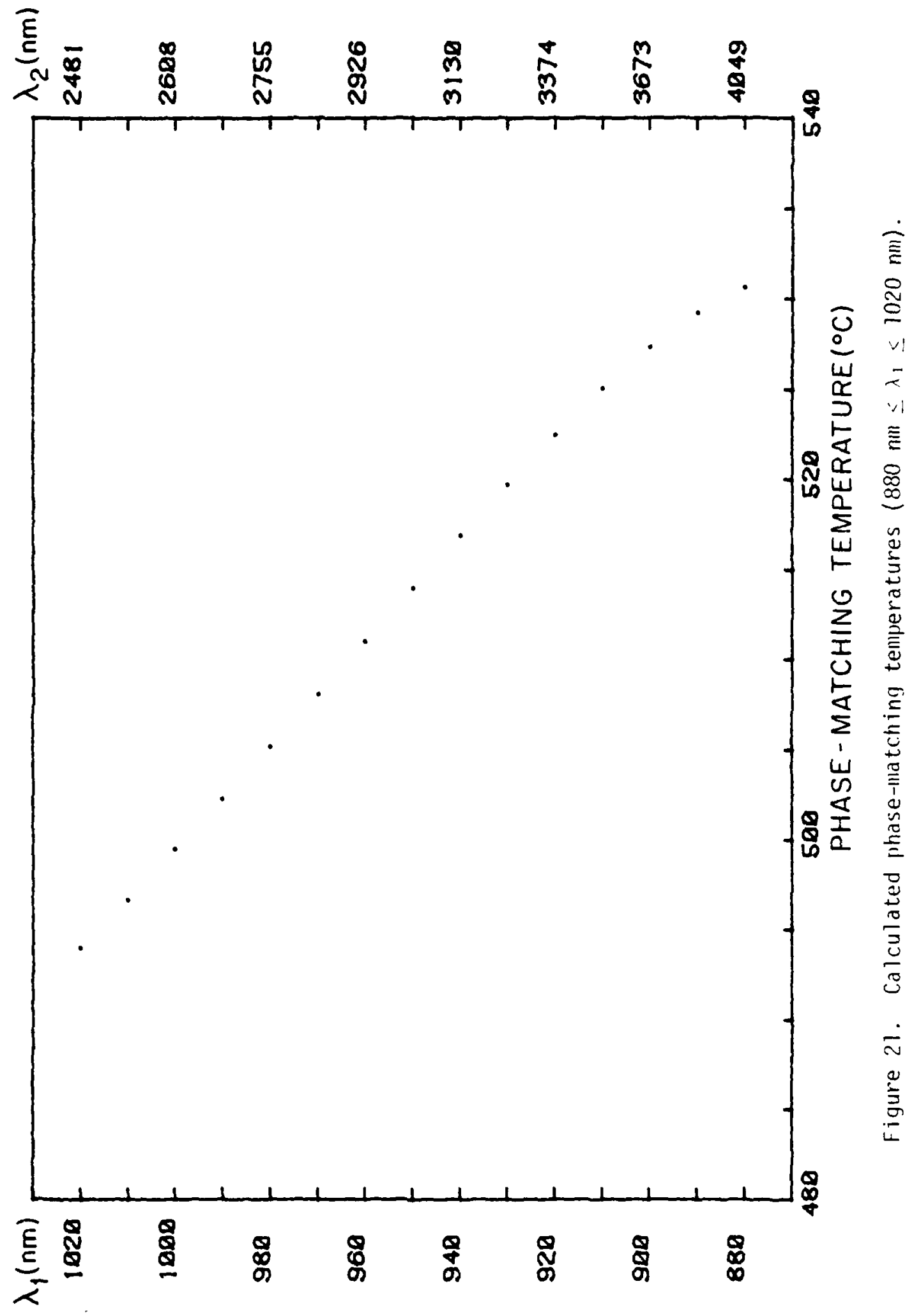


$-80-$

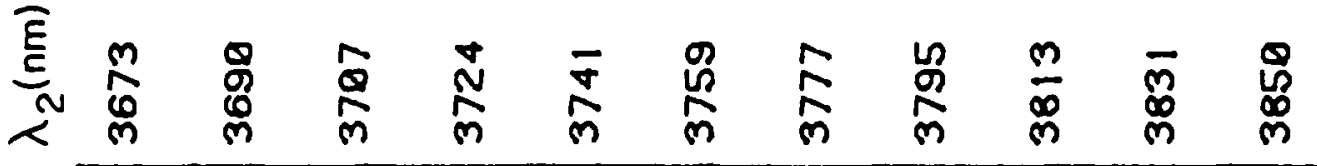

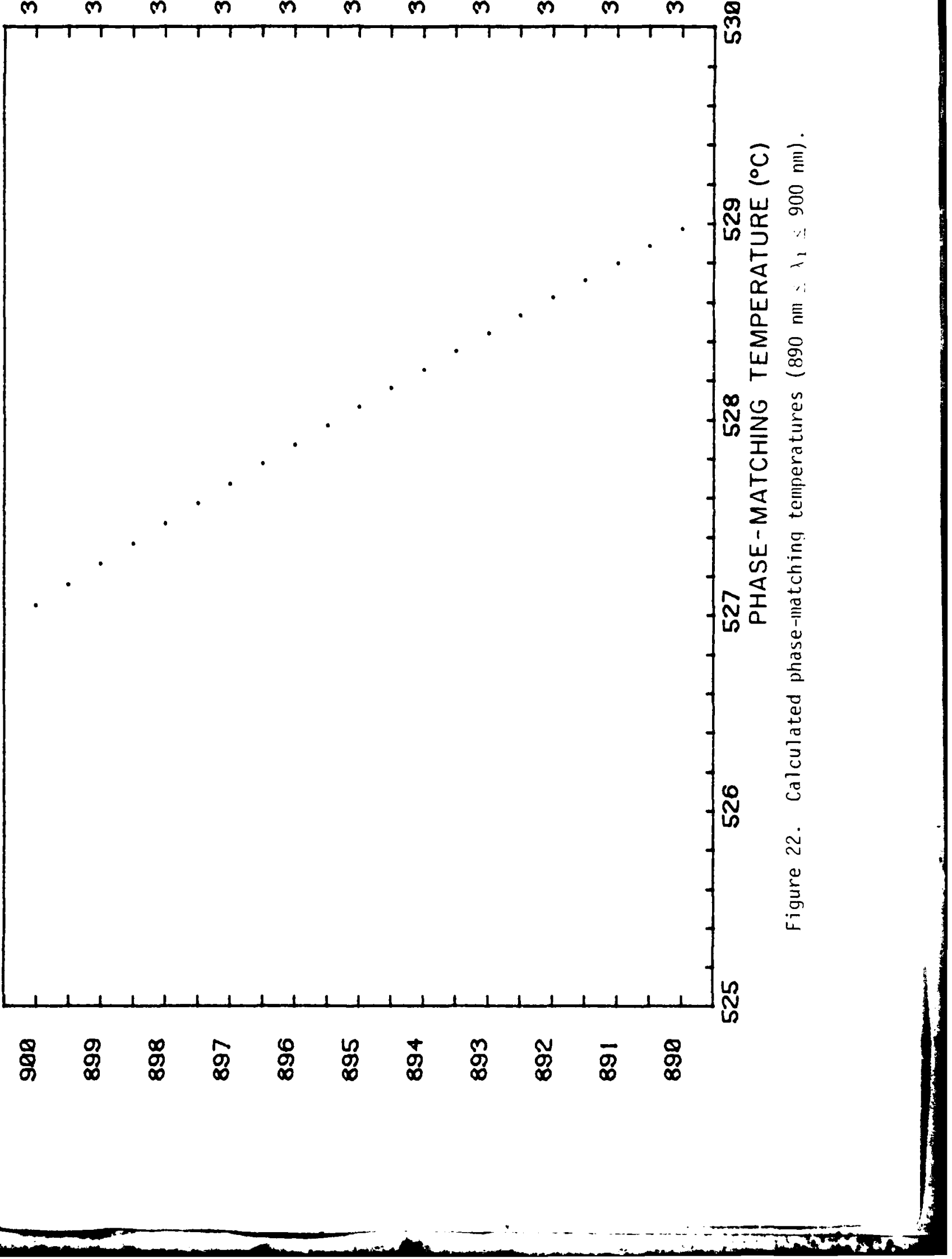


$1 k \leq T / 2$. At the end points of this range, the mixing efficiency is about $41 \%$ of the peak efficiency. A good estimate of the temperature bandwidth can be made by calculating the phase-matching temperature for a set of mixing frequencies using Eqns. 38 and 39 and then calculating $\operatorname{sinc}^{2}(j k, / 2)$ from Eqn. 39 as that temperature is varied. In Fig. 23 a graph of the relative mixing efficiency vs. the deviation from the phasematching temperature is shown. The data were calculated for the set of mixing frequencies for wnich $\lambda_{2}=940 \mathrm{~nm}$. The temperature bandwidth is approximately independent of wavelength over the desired mixing range and equal to $.64^{\circ} \mathrm{C}$. The oven temperature is controlled by a Thermologic 4011FS Proportional Controller with an Onega PT-100-K-20-15 Platinum Resistive Temperature Detector. It has been maintained to well within $.5^{\circ} \mathrm{C}$ for periods in excess of thirty minutes at temperatures around $500^{\circ} \mathrm{C}$.

To this point it has been assumed that the finite linewidth of the dye laser has a negligible effect on phase-inatching. To verify this, Eqns. 38 and 39 are used to calculate the approximate wavelength range over which $i k \leq \pi / 2$ for a number of dye laser wavelengths and the corresponding phase-matching temperatures. Table 3 is a list of these maximum allowable linewidths for efficient mixing. Note that the allowable linewidth exceeds $20 \AA$ across the desired mixing range. This is indeed much greater than the measured dye laser linewidth for either dispersive element. 


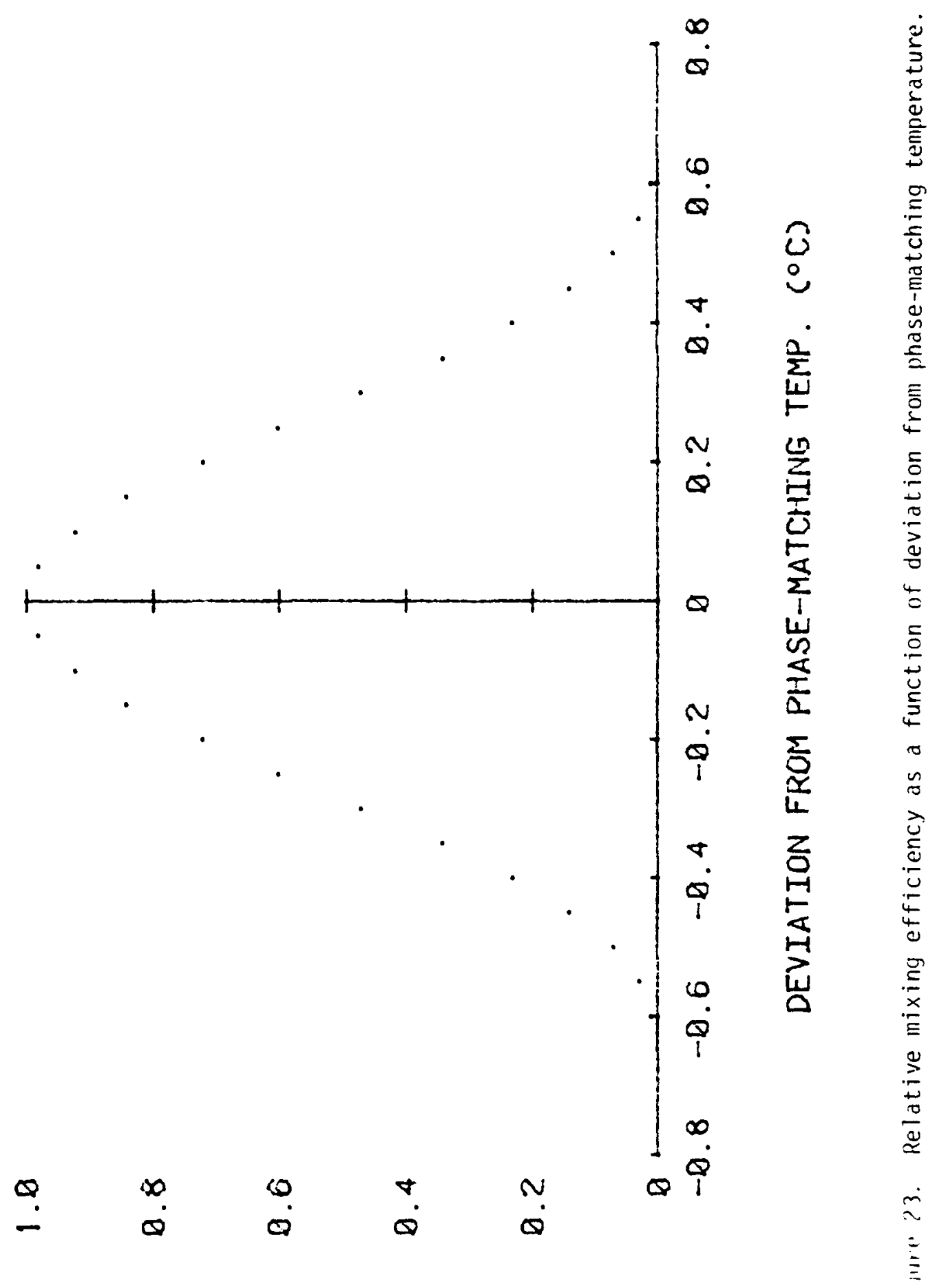

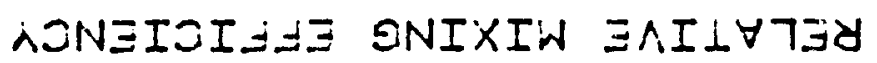




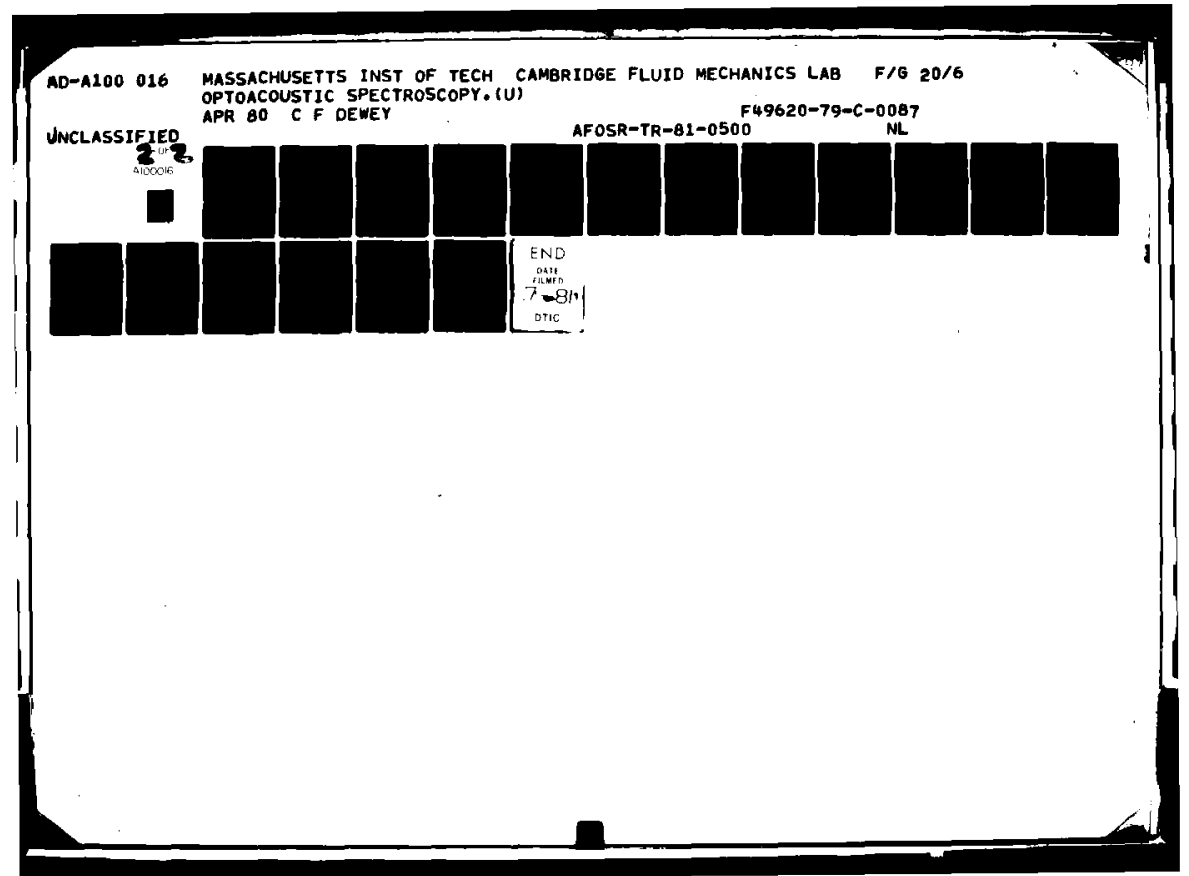


TABLE 3. MAXIMUM ALLOWABLE DYE LASER LINEWIDTHS

\section{FOR EFFICIENT MIXING $(\Delta k \leq \pi / 2)$}

\begin{tabular}{cc}
$\frac{\lambda_{2}(\mathrm{~nm})}{890}$ & $\frac{\Gamma_{\max }(\AA)}{36}$ \\
900 & 30 \\
910 & 26 \\
920 & 24 \\
930 & 22 \\
940 & 22 \\
950 & 22 \\
960 & 22 \\
970 & 22 \\
980 & 22 \\
990 & 22 \\
\hline
\end{tabular}

\subsection{The Optical System}

A schematic of the proposed difference-frequency generation optical system appears in Fig. 24. Positive lenses L1 and L2 focus the LVL and dye laser beams to the same point at the crystal. Beamsplitter BST divides the LVL beam; the reflected beam is focussed onto the dye cell to pump the dye laser and the transmitted beam pumps the lithium niobate crystal. The plane of polarization of the dye laser is rotated $90^{\circ}$ by the polarization rotator. The p-polarized LVL beam and the s-polarized dye laser beam are combined at the polarizing beamsplitter ES2. 


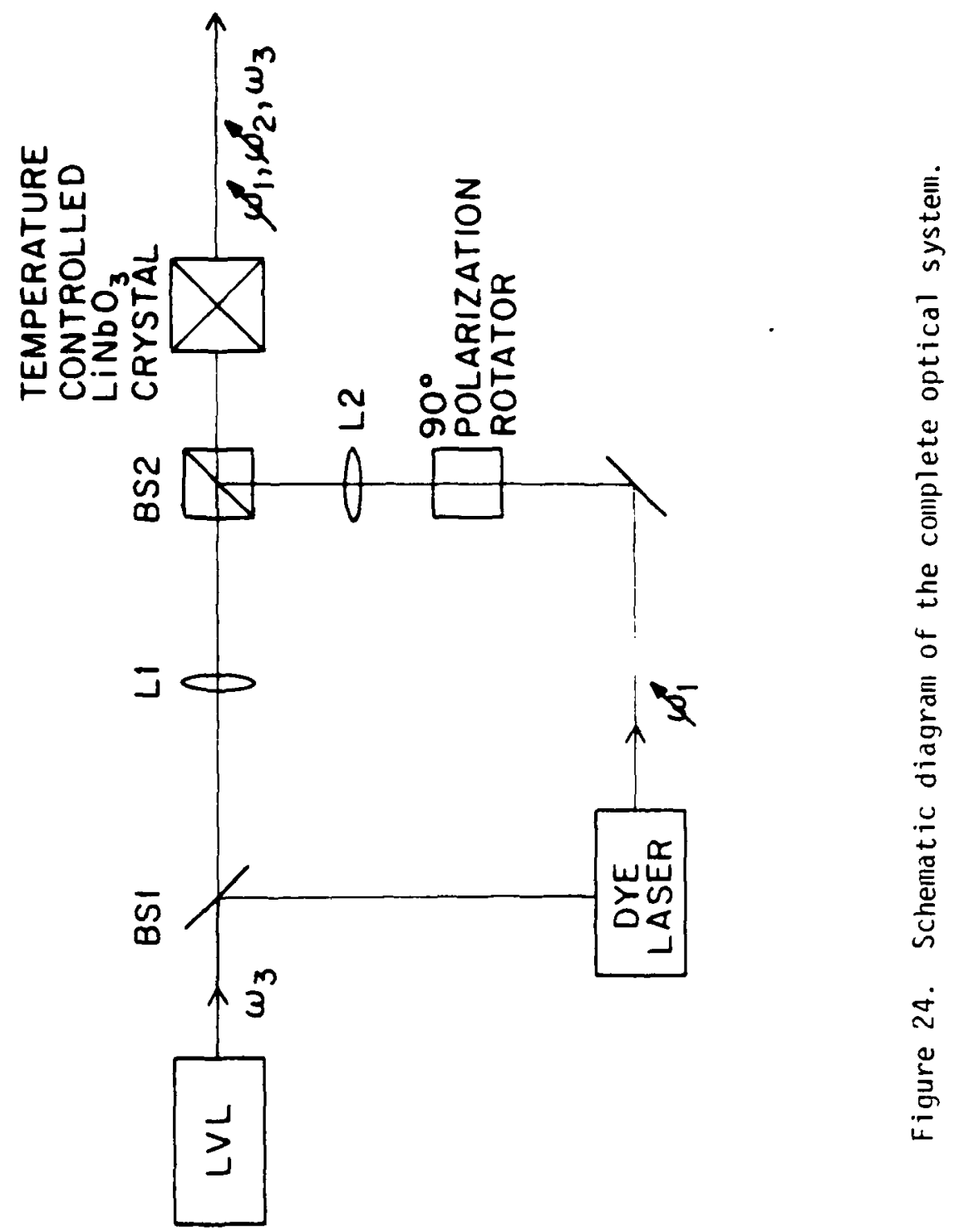


The crystal is oriented in the oven so that the LVL beam is polarized parallel to the $z$ (optic) axis of the crystal. (X-ray photographs have been taken to determine the correct crystal orientation.)

In order to determine the optimum ratio, $x$, of transmitted power to reflected power at BSI, the response of the dye laser is modeled by the following relation:

$$
P_{1}=A\left(\frac{X P_{3}}{P_{T}}-1\right)
$$

where $P_{1}$ is the dye laser output power; $P_{3}$ is the LVL power; $P_{T}$ is the threshold LVL pump power needed to operate the dye laser; and $A$ is a constant. The generated infrared power, $P_{2}$, is proportional to the product of the powers of the two mixing beams, $P_{1}$ and $(1-x) P_{3}$ :

$$
P_{2}=A^{\prime}\left(\frac{x P_{3}}{P_{T}}-1\right)(1-x) P_{3}
$$

where $A^{\prime}$ is a constant. The optimum value of $x$ is determined by taking the derivative of $P$ with respect to $x$ and setting it equal to zero:

$$
x_{O P T}=\frac{P_{3}+P_{T}}{2 P_{3}}
$$

Assuming $P_{3}=4 \mathrm{KW}$ and $P_{T}=1.3 \mathrm{KW}(40 \mu \mathrm{J}$ in $30 \mathrm{~ns})$ the optimum value is $x_{\text {OPT }}=.67$. 
The above mixing configuration is the conventional one for similar systems. The two mixing pulses occur with virtual simultaneity at the crystal. Typically, $P_{3} \gg P_{T}$ and optimization [Eq. (42)] requires that half of the pump power is used to excite the dye laser and half is used to stimulate the crystal. An alternative mixing configuration (Fig. 25) should result in greater mixing efficiency. In this system, the entire LVL beam stimulates the crystal. Because pump depletion is negligible in the mixing process, virtually all of the LVL beam then excites the dye laser. The simultaneity of the mixing pulses, though somewhat reduced in this system, may be maintained to within a few nanoseconds. As an example of the increase in efficiency, assume that the mixing pulses are $30 \mathrm{~ns}$ long and are separated at the crystal by $3 \mathrm{~ns}$ in time. Assume also that $P_{3} \gg P_{T}$. Then the reduced simultaneity will degrade the mixing efficiency by about $10 \%$ while the product of the mixing powers will increase by nearly a factor of four. The overall efficiency would be about 3.6 times that of the conventional arrangement.

\subsection{Theoretical Conversion}

In this Section the theoretical infrared power, $P_{2}$, that could be generated at $\lambda_{2}=3.4 \mathrm{jm}$ by this system is calculated. For convenience, Eq. 9, the equation for the power generated in a phasematched crystal in MKS units, is repeated here from Ch. 2 in terms of commonly encountered units: 
$-87-$

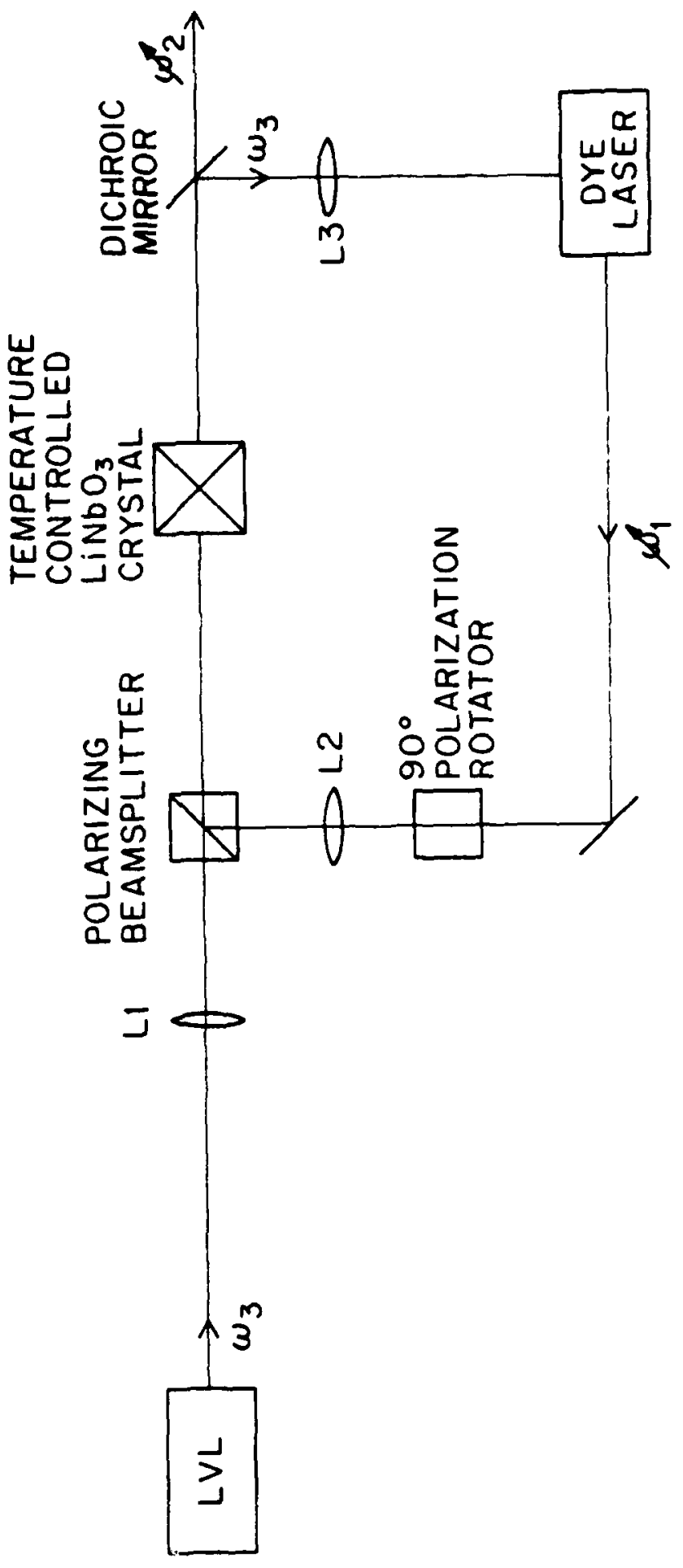

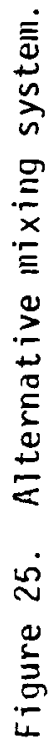




$$
P_{2}=\frac{\left(5.3 \times 10^{-3}\right) \omega_{2}^{2} d^{2} \ell^{2} P_{3} P_{1}}{n_{1} n_{2} n_{3}\left(W_{3}^{2}+W_{1}^{2}\right)} \cdot\left[T_{1} T_{2} T_{3} \exp \left(-\frac{\alpha_{2} \ell}{2}\right)\right]
$$

where $d$ is in meters per volt $t^{\star}, \ell$ is in millimeters; $P$ is in kilowatts; and $W$ is in microns.

The beam waists, $w_{0}$, of the focussed LVL and dye laser beanis are assumed to be $50 \mu \mathrm{m}$ in the mixing crystal. For waists of this size at $\lambda_{3}=.7229 \mu \mathrm{m}$ and $\lambda_{1}=.918 \mu \mathrm{m}$, the length of the focal region, $2 \pi \mathrm{w}_{0}{ }^{2} \mathrm{n} / \lambda$ (defined as the region over which the l/e radius of the Gaussian field is less than $\left.\sqrt{2} \cdot w_{0}\right)$, is greater than $10 \mathrm{~cm}$. Therefore, the beam areas are approximately constant over the length of the crystal ( $\mathrm{l}=10 \mathrm{~mm}$ ) and the spot sizes are $W_{3}=W_{1}=W_{0}=50 \mu \mathrm{m}$. Complete spatial overlap of the two beam: within the crystal is assumed.

The appropriate values for the refractive indices are calculated from Eqns. 39 . These values are used to determine the relevant transmission coefficients at the crystal faces where

$$
T=\frac{4 n}{(n+1)^{2}}
$$

The value of the effective nonlinear coefficient for $90^{\circ}$ phasematching is taken from Boyd and Kle inman 41 to be $d=d_{31}=d_{15}=$ $6.3 \times 10^{-12} \mathrm{~m} / \mathrm{V}$. The absorption of the lithium niobate crystal over $1 \mathrm{~cm}$ is negligible at the wavelengths involved for this calculation

\footnotetext{
$\frac{d(M K S ; m / v)}{d(e s u ; c m / s t a t v o l t)}=4.2 \times 10^{-4}$
} 
and reflection losses at surfaces in the optical system are ignored. Assuming that the LVL produces 120 iJ pulses of $30 \mathrm{~ns}$ width and that half of the beam pumps the dye laser $(x=.5)$ at $5 \%$ power efficiency, the peak power generated at $\lambda_{2}=3.4 \mu \mathrm{m}$ will be

$$
P_{2 \text { peak }}=15 \mathrm{~W}
$$

This corresponds to a conversion efficiency for LVL power to infrared power of .38\%. The average power at $2 \mathrm{KHz}$ PRF for $20 \mathrm{~ns}$ infrared pulses will be

$$
P_{2} \text { average }=.61 \mathrm{~mW}
$$




\section{CONCLUSION}

The infrared difference-frequency system is essentially complete but untested. The mixing sources, the lead vapor laser and the dye laser, are operational. The dye laser linewidth and the crystal temperature control necessary for efficienct mixing has been demonstrated. To evaluate the state of this system, and to suggest areas for further research, the system is again examined in terms of its three primary components:

The Lead Vapor Laser - The LVL can be operated for tens of hours with peak output pulse energies in excess of $100 \mu \mathrm{J}$. The pulsewidth is about $30 \mathrm{~ns}$ and pulse repetition rates of up to $2 \mathrm{KHz}$ have been demonstrated.

The key to evaluating this laser from the point of view of difference-frequency mixing may be the mode quality of the beam. By employing the barbell tube, the mode quality and lifetime have been substantially upgraded. By using a relatively high reflectivity output coupler $(R=.35)$, the mode quality has been further enhanced, but at the expense of output power. In terms of infrared power generation, this appears to be a valid trade-off. This issue must be examined when the mixing system is tested.

The issues of mode quality and efficiency of the LVL are related by more than the choice of output coupler. Evidence of non-uniformities in the discharge appear in the output beam and the result is incomplete excitation of the lead atoms by the discharge. The uniformity of the 
discharge is possibly a function of the electrode configuration and may be affected by such things as liquid lead in the discharge tube and magnetic fields generated during the sub-microsecond current pulse. These are areas where investigation is crucial but quite difficult. However, even a rough qualitative understanding in these areas could be very important.

The Dye Laser - The dye Taser energy efficiency exceeds $1 \%$ and the LVL pump pulse threshold is less than $40 \mathrm{uJ}$. The dye laser z-cavity is useful for optically-pumped lasers where extremely low pump energies are available and has been analyzed in detail.

One dye, IR-140, has been used in the dye laser to produce radiation in the desired mixing range. It was tunable over close to $30 \mathrm{~nm}$ and, with a diffraction grating in the cavity, had a linewidth narrower than $1 \AA$. A number of infrared dyes remain to be tested in the laser to extend the tuning range up to wavelengths of at least $1 \mu \mathrm{m}$. The operation of the dye laser with each dye must be carefully characterized.

The outstanding issue of the dye laser is the relatively low efficiency. A factor of five improvement is anticipated here. A number of factors that may be affecting the efficiency were discussed in Section 4.6. Each of these should be considered in order to ameliorate this probiem.

The Mixing System - The crystal temperature control necessary for efficient mixing has been calculated and demonstrated. The method of spontaneous parametric scattering can be used to determine the temperature 
correction to the Hobden-Warner equations for the crystal. Once this is done, the phase-matching temperatures for infrared wavelengths up to 4 um can be calculated quite accurately.

The final selection of optics for the mixing system will require some experimental evaluation. It is dependent on the LVL mode quality and the practical problems involved in achieving the high degree of spatial overlap required between the two beams in the mixing crystal. The LVL has been focussed to a beam waist smaller than $50 \mu \mathrm{m}$ but its propagation behaviour deviates significantly from that of an ideal Gaussian beam. The same is true to some extent of the dye laser although the nonideality is moderated by the higher ratio of pulse width to cavity length and the lower gain. The net effects of the mode quality of the LVL are the limitation of the obtainable spot size and the constriction of the focal region for a given beam waist. These problems make it difficult to calculate the appropriate focal lengths for the lenses in the mixing system and require some experimental determinations to be made.

The alternative mixing configuration suggested in Section 5.3 (Fig. 25) is promising, especially in light of the small difference in LVL and dye laser pulse widths observed at higher pump energies. This system warrants serious investigation as it offers a significant increase in efficiency over the conventional arrangement.

In the work covered by this thesis, a number of practical difficulties involved in this infrared difference-frequency generation system 
have been uncovered. At the same time, the source lasers have been developed to a useful point and the entire mixing process has been analyzed and deemed to be feasible. The advantages of generating tunable, narrow linewidth infrared radiation by this means remain. A continuation of this effort is suggested before judgment is passed on this system. 


\title{
APPENDIX A. DETECTIVITY OF CRYSTAL SPONTANEOUS
}

\author{
PARAMETRIC EMISSION AND CRYSTAL CALIBRATION
}

Spontaneous parametric scattering can be induced by pumping the lithium niobate crystal in the temperature-controlled oven with the LVL beam aione. Under phase-matched conditions, this pump field will mix with a virtual field within the crystal to produce difference-frequency radiation. The crystal must be oriented in the oven as it would be in the difference-frequency arrangement such that the plane-polarized pump beam propagates as an extraordinary ray. An anti-reflection-coated silicon filter placed between the crystal oven and a sensitive infrared detector (indium antimonide) will block the LVL beam and pass any spontaneously emitted infrared radiation. This filter is available here as are three infrared notch filters between $3 \mathrm{um}$ and $4 \mathrm{um}$ that can be used before the detector to determine the wavelength of the infrared emission. A chromel-alumel thermocouple embedded in the crystal mount within the oven can be used to find the phase-matching temperature of the emission of known wavelength. In this way the offset temperature can be determined and the crystal can be calibrated with respect to the Hobden-Warner equations. A list of available infrared notch filters including the center wavelength $\lambda_{c}$, the bandwidth $j \lambda$, and the peak transmission $T_{p}$, is shown in Table A.1. The fourth filter at $4.3 \mathrm{jm}$ can be used to calibrate the crystal at a wavelength just beyond the stated range of the Hobden-Warner equations. 
TABLE A.1. NOTCH FILTERS

\begin{tabular}{lcr}
$\lambda_{c}(\mu \mathrm{m})$ & $\Delta \lambda(\mu \mathrm{m})$ & $T_{p}$ \\
\hline 3.125 & .155 & .70 \\
3.398 & .116 & .84 \\
3.427 & .147 & .83 \\
4.286 & .405 & .80 \\
\hline
\end{tabular}

In order to verify the detectability of the infrared spontaneous parametric emission, the minimum detectable power of the detector and the expected infrared emission power are calculated. The minimum detectivity of the available Philco ISC-3010 Indium Antimonide Detector from $3-5 \mathrm{um}$ is $D^{*}=1.5 \times 10^{10} \mathrm{~cm} \mathrm{~Hz} \frac{1}{2} / \mathrm{W}$ and the detector area is $A=4.1 \times 10^{-2} \mathrm{~cm}^{2}$. It is used in conjunction with a Perry Amplifier Model 720 Automatic Bias Control Amplifier that has a bandwidth of $\Delta f=1 \mathrm{iHz}$. The noise effective power (N.E.P.) for this combination is:

$$
\text { N.E.P. }=\frac{(A \cdot \Delta f)^{\frac{1}{2}}}{D^{*}}=1.3 \times 10^{-8} \mathrm{~W}
$$

Assuming a 30 ns laser pulse the minimum detectable power $(S / N=1)$ is $4.5 \times 10^{-7} \mathrm{~W}$.

To find the emitted infrared signal power, the following relation found by Byer and Harris 40 is used: 


$$
P_{S}=\left(\beta \ell, P_{p} / b\right) \pi \theta^{2}
$$

where $P_{s}$ and $P_{p}$ are the infrared and pump powers, respectively; $B$ is a constant that is related to the interaction strength; $\ell$ is the crystal length; $b$ is an angular dispersive constant; and $\theta$ is the acceptance angle in radians into which infrared power is generated. It is important to note that, unlike the case of difference-frequency generation, $P_{S}$ is independent of the pump intensity within the crystal. For $\lambda_{s}=3.4 \mu \mathrm{m}$ (corresponding to the second notch filter listed in Table 4), the constant values are calculated to be $\beta=8.3 \times 10^{-16} \mathrm{sec} \cdot \mathrm{m}^{-2}$ and $\mathrm{b}=$ $1.3 \times 10^{-10} \mathrm{sec} \cdot \mathrm{m}^{-1}$ ( $b$ is found using Eqns. 39). The crystal length is $\ell=10 \mathrm{~mm}$. Assuming a pump pulse energy of $120 \mathrm{wJ}$ in $30 \mathrm{~ns}\left(P_{p}=4 \mathrm{KW}\right)$ and taking into account the transmission of the silicon filter $(T=.95)$ and the notch filter $(T=.84)$, the signal power as a function of $\theta$ is:

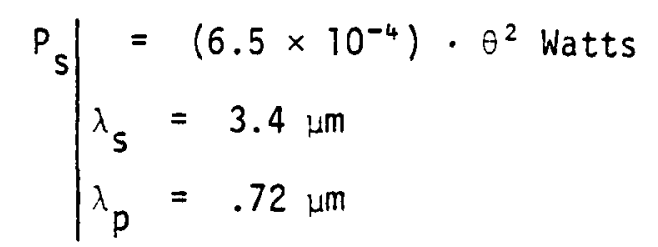

Evidently an acceptance angle of $\theta=1.5^{\circ}$ is required for a signal to noise ratio of $S / N=1$. The acceptance angle is limited by the oven geometry. The crystal oven has an inside diameter of .56" and is 5.5" long. Therefore the maximum acceptance angle is $\theta_{\max }=5.8^{\circ}$ and the spontaneous parametric emission in the infrared should be quite detectable. 
The accuracy of the experimentally-determined offset temperature is limited by the bandwidth of the notch filter. The 3.4 um filter has a bandwidth of $\Delta \lambda=.116 \mu \mathrm{m}$. In order to find the variation in the phase-match temperature across the spectral bandpass region of the filter Eqns. 38 and 39 are used. The calculated variation in phasematching temperature indicates that the experimentally determined offset temperature will be within $1.1^{\circ} \mathrm{C}$ of the actual value. 


\section{REFERENCES}

1. Optoacoustic Spectroscopy and Detection, Edited by Yoh-Han Pao, Academic Press, N. Y., 1977

2. J. Kuhl and W. Schmidt, Applied Physics, Vol. 3, No. 4, pp. 251270, April, 1974

3. D. C. Hanna, R. C. Smith, and C. R. Starky, Optics Communications, Vol. 4, No. 4, pp. 300-303, December, 1971

4. A. Mooradian, Reports on Progress in Physics, Vol. 42, pp. 1533. 1564,1979

5. C. F. Dewey and L. 0. Hocker, Appl ied Physics Letters, Vol. 18, No. 2, pp. 58-60, January 15, 971

6. D. W. Meltzer and L. S. Goldberg, Optics Communications, Vol. 5, No. 3, pp. 209-211, June, 1972

7. L. S. Goldberg, Applied Optics, Vol. 14, No. 3, pp. 653-656, March, 1975

8. H. Tashiro and T. Yajima, Optics Communications, Vol. 12, No. 2, pp. 129-131, October, 1974

9. C. G. Bethea, Applied Optics, Vol. 12, No. 6, pp. 1104-1105, June, 1973

10. C. D. Decker and F. K. Tittel, Applied Physics Letters, Vol. 22, No. 8, pp. 411-413, April 15, 1973

11. C. D. Decker and F. K. Tittel, Optics Communications, Vo1. 8, No. 3, pp. 244-247, July, 1973

12. L. 0. Hocker and C. F. Jewey, Applied Physics, Vol. 11, No. 2, pp. 137-140. October, 1976

13. R. J. Seymour and F. Zernike, Applied Physics Letters, Vol. 29, No. 11, pp. 705-707, October, 1976

14. H. Gerlach, Optics Communications, Vol. 12, No. 4, pp. 405-408, December, 1974

15. A. S. Pine, Journal of the Optical Society of America, Vo1. 64, No. 12, pp. 1683-1690, December, 1974

16. W. Lahmann, K. Tibulski, and H. Welling, Optics Communications, Vol. 17, No. 1, Dp. 18-20, April, 1976 
17. R. L. Byer, M. M. Choy, R. L. Herbst, D. S. Chemla, and R. S. Fiegelson, Applied Physics Letters, Vol. 24, No. 2, pp. 65-68, January 15,1974

18. D. C. Hanna, B. Luther-Davies, R. C. Smith, and R. Wyatt, Applied Physics Letters, Vol. 25, No. 3, pp. 142-144, August 1, 1974

19. D. Andreov, Optics Communications, Vol. 23, No. 1, pp. 37-43, October, 1977

20. A. Bianchi, A. Ferrario, and M. Musci, Optics Communications, Vol. 25, No. 2, pp. 256-258, May, 1978

21. D. W. Feldman, C. S. Liu, J. L. Pack, and L. A. Weaver, Journal of Applied Physics, Vol. 4ý, No. 7, pp. 3679-3683, July, 1978

22. A. A. Isaev and G. G. Petrash, Journal of Experimental and Technical Physics Letters, Vol. 10, No. 4, pp. 119-121, August, 1969

23. L. O. Hocker, J. H. Flint, M. Madigan, and C. F. Dewey, Temperature Dependence of the Infrared Laser Lines in Lead Vapor, unpublished

24. R. S. Anderson, B. G. Bricks, T. W. Karras, and L. W. Springer, IEEE Journal of Quantum Electronics, Vol. QE-12, No. 5, pp. 313-315, May, 1976

25. C. R. Vidal and J. Cooper, Journal of Applied Physics, Vol. 40, No. 8, p. 3370, July, 1969

26. R. T. Hodgson, "Vapor Discharge Cel1", U.S. Patent 3,654,567, issued Apri1 4, 1972

27. R. Burnham and N. Djeu, Optics Letters, Vo1. 3, No. 6, pp. 215-217, December, 1978

28. D. W. Feldman, private communication

29. T. W. Hänsch, Applied Optics, Vol. 11, No. 4, pp. 895-898, Apri1, 1972

30. H. Kogelnik, Bell System Technical Journal, Vol. 44, No. 3, pp. 455494, March, 1965

31. H. Kogelnik, E. Ippen, A. Dienes, and C. Shank, IEEE Journal of Quantum Electronics, Vol. QE-8, No. 3, pp. 373-379, March, 1972

32. Optical Properties of Materials, Vol. 1, A. Moses, IFI/Plenum, N. Y., 1971, p. 68 
33. Optics, E. Hecht and A. Zajac, Addison-Wesley Publishing Co., Reading, MA, 1975, pp. 129-131

34. P. K. Runge and R. Rosenberg, IEEE Journal of Quantum Electronics, Vol. QE-8, No. 12, pp. 910-911, December, 1972

35. W. W. Morey, Technical Report, AFAL-TR-78-84, September 1, 1978. Air Force Avionics Laboratory (DHO), Wright-Patterson AFBase, Ohio 45433

36. $1,1^{\prime}, 3,3,3^{\prime}, 3^{\prime}$-Hexamethylindotricarbocyanine Perchlorate, Eastman Organic Chemicals Catalog No. 15070

37. 5,5'-Dichioro-11-diphenylamino-3,3'-diethyl-10,12-ethylene thiatricarbocyanine Perchlorate, Eastman Organic Chemicals Catalog No. 14402

38. G. M. Zverev, E. A. Levchuk, V. A. Pashkov, and Yu. D. Poryadin, Soviet Journal of Quantum Electronics, Vol. 2, No. 2, pp. 167-169, September-October, 1972

39. M. V. Hobden and J. Warner, Physics Letters, Vol. 2?, No. 3, pp. 243-244, August 15, 1966

40. R. L. Byer and S. E. Harris, Physical Review, Vol. 168, No. 3, pp. 1064-1068, Apri\} 15, 1968

4:1. G. D. Boyd and D. A. Kleinman, Journal of Applied Physics, Vol. 39, No. 8, pp. 3597-3637, July, 1968 


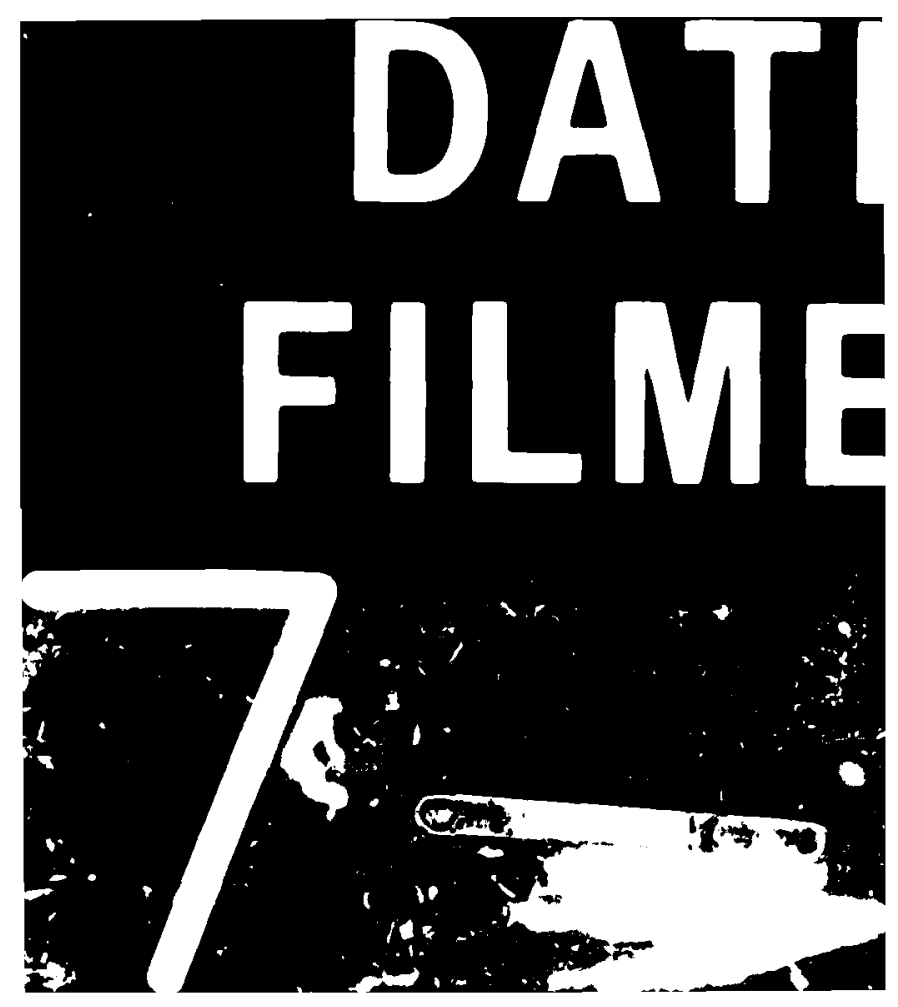

